University of Louisville

ThinkIR: The University of Louisville's Institutional Repository

Electronic Theses and Dissertations

$5-2020$

\title{
Optimization and simulation models to improve access to organ transplantation in the United States.
}

\author{
Fatemeh Karami \\ University of Louisville
}

Follow this and additional works at: https://ir.library.louisville.edu/etd

Part of the Industrial Engineering Commons, and the Operational Research Commons

\section{Recommended Citation}

Karami, Fatemeh, "Optimization and simulation models to improve access to organ transplantation in the United States." (2020). Electronic Theses and Dissertations. Paper 3379.

https://doi.org/10.18297/etd/3379

This Doctoral Dissertation is brought to you for free and open access by ThinkIR: The University of Louisville's Institutional Repository. It has been accepted for inclusion in Electronic Theses and Dissertations by an authorized administrator of ThinkIR: The University of Louisville's Institutional Repository. This title appears here courtesy of the author, who has retained all other copyrights. For more information, please contact thinkir@louisville.edu. 


\title{
OPTIMIZATION AND SIMULATION MODELS TO IMPROVE ACCESS TO ORGAN TRANSPLANTATION IN THE UNITED STATES
}

\author{
By \\ Fatemeh Karami \\ M.Eng., Iran University of Science and Technology, Iran, 2014 \\ B.Eng., Khaje Nasir University of Technology, Iran, 2011
}

\begin{abstract}
A Dissertation
Submitted to the Faculty of the

J. B. Speed School of Engineering of the University of Louisville in Partial Fulfillment of the Requirements for the Degree of
\end{abstract}

Doctor of Philosophy in Industrial Engineering

\author{
Department of Industrial Engineering \\ University of Louisville \\ Louisville, Kentucky
}

May 2020 



\title{
OPTIMIZATION AND SIMULATION MODELS TO IMPROVE ACCESS TO ORGAN TRANSPLANTATION IN THE UNITED STATES
}

\author{
By \\ Fatemeh Karami \\ M.Eng., Iran University of Science and Technology, Iran, 2014 \\ B.Eng., Khaje Nasir University of Technology, Iran, 2011
}

A Dissertation submitted on

May 5, 2020

to the following Dissertation Committee:

Professor Monica Gentili, Dissertation Director

Professor Lihui Bai

Professor John S. Usher

Professor Alexander Suraj

Professor Adel Elmaghraby 


\section{ACKNOWLEDGEMENTS}

First of all, I would like to express my sincere gratitude to my advisor Dr. Monica Gentili for her support, patience, guidance, and for everything she has taught me throughout the tenure of my Ph.D. study. Without her support this dissertation would have not been completed. Her hard working attitude and passion were an inspiration I will carry throughout my career.

I am gratefull to all of those with whom I have had the pleasure to work during this and other related projects. I would love to express my deepest gratitude to Dr. Naoru Koizumi for being an amazing friend, a great mentor, and providing a new perspective on the subject. Also, I thank Dr. Sommer Gentry for sharing her knowledge in organ transplantation and giving me her kind support on personal and professional level. Additionally, I am very grateful for working with Dr. Sean Barnes and everything that he has thought me about which added so much value to my work. I also appreciate the help that I have received in simulation modeling from Dr. Micheal Fu, and his support and availability even in his busiest days. For me, one of the great outcome from this project was getting to know these great professors, learning from them and calling them my friends.

Moreover, I would like to thank my other committee members Dr. Alexander Suraj, Dr. Lihui Bai, Dr. John Usher, and Dr. Adel Elmaghraby who have been supportive of my work and provided valuable comments, suggestions and guidance during the course of this dissertation. I also love to express my gratitude to the Department of Industrial Engineering for the financial support.

I am very thankful for my amazing friends Amir, Aida and Reihaneh, for their great friendship and support from the time I was an undergrad student till 
now. I would love to thank my great friends in Louisville, Ankita, Mina, Hannah, Luis, Gang, and Parag, who made my life in Louisville fun and gave me the motivation to go to the office everyday. Additionally, I appreciate the support that I have received from my friends in ERGOT lab at The Johns Hopkins University. Nobody has been more important to me to pursuit this project than the members of my family. I would love to thank my parents, Zarintala and Gholamreza, for their love. Their guidance is with me in whatever I pursue. Most importantly, I would love to thank my loving boyfriend Daniele, for his warm love and endless support. 


\begin{abstract}
OPTIMIZATION AND SIMULATION MODELS TO IMPROVE ACCESS TO ORGAN TRANSPLANTATION IN THE UNITED STATES
\end{abstract}

Fatemeh Karami

May 5, 2020

Organ allocation in the U.S. is administrated by the United Network of Organ Sharing (UNOS). UNOS's mission is to ensure fair and equitable allocation of organs as stated in the Code of Federal Regulations, which reads "neither place of residence nor place of listing shall be a major determinant of access to transplant". Despite the regulations, there has been endless controversy surrounding the disparity in access to organ transplants. In this context, the primary research goal in this dissertation was to reduce geographic disparity in access to transplants in the U.S., with a focus on heart and kidney transplants.

To improve access to heart transplants, we first analyzed the status of geographic disparity and organ utilization under current practice. Next, we used survival analysis and statistical analysis to measure heart utilization rate across the country and studied the factors that can improve heart usage. Additionally, we defined a novel optimization model to modify the geographic boundaries in the U.S. heart allocation system. Finally, We developed a clinically detailed discrete event simulation model for the U.S. heart allocation system to evaluate our proposed changes in in the heart allocation policy stemming from the optimization model.

To improve access to kidney transplants, first, we proposed a simulation - 
optimization approach for better utilization of donated kidneys from living donor through a Kidney Paired Donation (KPD) program. Additionally, to reduce geographic disparity in access to kidney transplants, we used an optimization model to redesign geographic boundaries in the kidney allocation system.

Our findings indicated that using optimization and simulation models can greatly improve equity in access to organ transplants. 


\section{TABLE OF CONTENTS}

ACKNOWLEDGEMENTS

ABSTRACT $\quad$ V

LIST OF TABLES $\quad$ x

LIST OF FIGURES X xiii

I INTRODUCTION 1

A The Big Challenge: Geographic Disparity in Access to Transplants 5

II ANALYZING ACCESS TO HEART TRANSPLANTS 10

A Analyzing Geographic Disparity in Access to Heart Transplants 10

$1 \quad$ Introduction . . . . . . . . . . . . . . . 10

2 Methods and Materials . . . . . . . . . . . . . 14

3 Results .................. 17

4 Conclusion . . . . . . . . . . . . . . . 32

B Transplant Outcome of Adult Heart Donor with Prior Qualitybased Offer Refusal . . . . . . . . . . . . . . . . . 34

$1 \quad$ Study Population . . . . . . . . . . . . . 36

2 Geographic Disparity in Offer Heart Refusal . . . . . . 36

3 Discarded Hearts Rate . . . . . . . . . . . . . 37

$4 \quad$ Transplant Outcome for Hearts with Prior Offer Refusal . 38

5 Conclusion ................... 47

III OPTIMIZATION MODEL TO ADDRESS GEOGRAPHIC DISPARITY IN ACCESS TO HEART TRANSPLANTS 48

A Introduction . . . . . . . . . . . . . . . . 48 
B Problem Definition . . . . . . . . . . . . . . . 52

1 Zonal Structure Definition . . . . . . . . . . . . . 52

2 Access Level . . . . . . . . . . . . . . . . . . 53

3 Access Level Disparity _. . . . . . . . . . . . . 57

4 The Boundary Allocation Problem . . . . . . . . . . 57

C Model Formulation . . . . . . . . . . . . . . . . 58

D Weight Tuning . . . . . . . . . . . . . . 60

E Experimental Analysis . . . . . . . . . . . . . . . 63

$1 \quad$ Data . . . . . . . . . . . . . . . 63

2 Scenario Description . . . . . . . . . . . . 64

F Results ................... 66

$1 \quad$ Access Level Disparity _. . . . . . . . . . . . . . . 66

2 Zonal Structure Heterogeneity . . . . . . . . . . . 67

3 Distance Traveled by Organs . . . . . . . . . . . . . . 69

G Sensitivity Analysis . . . . . . . . . . . . . . 70

$1 \quad$ Supply and Demand . . . . . . . . . . . . . 70

2 Maximum Circle Radius . . . . . . . . . . . . . 72

H Discussion . . . . . . . . . . . . . . . . . . . 74

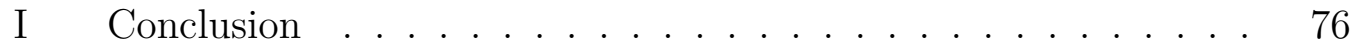

IV HEART ALLOCATION SIMULATION MODEL 78

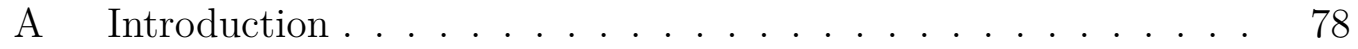

B Heart Allocation System . . . . . . . . . . . . . . . 79

C Simulation Framework . . . . . . . . . . . . . 80

$1 \quad$ Patient Arrival . . . . . . . . . . . . 80

2 Patient Status Update . . . . . . . . . . . . . 81

$3 \quad$ Organ Arrival . . . . . . . . . . . . . . . . . 82

4 Post Transplant Graft Survival . . . . . . . . . . . . 83

5 Simulation Outcomes . . . . . . . . . . . . . . 84 
D Model Validation . . . . . . . . . . . . . . . . 85

E Heterogeneous vs Homogeneous Zonal Structure . . . . . . . . 86

F Conclusion . . . . . . . . . . . . . . . . . . . 89

V OPTIMAL INTEGRATION OF DESENSITIZATION PROTOCOLS INTO KIDNEY PAIRED DONATION (KPD) PROGRAMS 92

A Introduction . . . . . . . . . . . . . . . . 92

B Optimization Model . . . . . . . . . . . . . . . . 95

C Experimental Analysis . . . . . . . . . . . . . . . . . . 99 99

$1 \quad$ Data . . . . . . . . . . . . . . . . . . . 99

$2 \quad$ Scenarios . . . . . . . . . . . . . . . . . . 99

$3 \quad$ Simulation Strategy . . . . . . . . . . . . . . . . . . . 101

$4 \quad$ Output Measures . . . . . . . . . . . . . . . 103

D Results ........................ . . 103

E Conclusion .................... . 110

VI ALLOCATING KIDNEYS IN OPTIMIZED HETEROGENEOUS CIRCLES 116

A Introduction . . . . . . . . . . . . . . 116

B Method ....................... 117

C Results ....................... . 119

D Sensitivity Analysis . . . . . . . . . . . . . . . . 120

E Discussion . . . . . . . . . . . . . . . . . . . 121

$\begin{array}{ll}\text { VII SUMMARY AND CONCLUSION } & 128\end{array}$

REFERENCES 132

$\begin{array}{ll}\text { CURRICULUM VITAE } & 157\end{array}$ 


\section{LIST OF TABLES}

1 Adult heart allocation (2005 to present) (Meyer, 2015) . . . . . . 13

2 Patient status classification for from 1998 to present and approved in

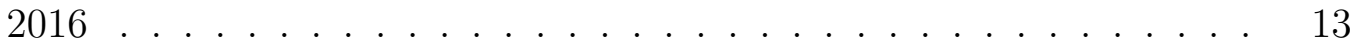

3 Heart allocation scheme for adult candidates approved in 2016 (2016 Policy) . . . . . . . . . . . . . . . . . . . . . . . . . . 14

4 Median Waiting Time (days): average and median values at the TC, DSAs and the region level for different urgency statuses while on the waiting list . . . . . . . . . . . . . . . . . . . 18

5 Median Waiting Time: disparity metrics at the TC level, DSA level and region level for different urgency statuses while on the waiting list

6 Transplant Rate: average and median values at the TC, DSA and region level for different urgency statuses while on the waiting list .

7 Median Waiting Time: disparity metrics at the TC level, DSA level and region level for different urgency statuses while on the waiting list

8 Pre-Transplant Mortality Rate: average and median values at the TC, DSA and region level for different urgency statuses while on the waiting

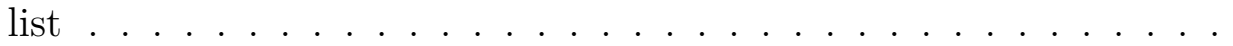

9 Pre-Transplant Mortality Rate: disparity metrics at the TC, DSAs and the region level for different urgency statuses while on the waiting list

10 Average Distance Traveled by Donated Hearts: average and median values at the TC, DSAs and the region level for different urgency statuses while on the waiting list $\ldots \ldots \ldots \ldots \ldots$ 
11 Average Distance Traveled by Donated Hearts: disparity metrics at the TC, DSAs, and region level for different urgency statuses while on the waiting list . . . . . . . . . . . . . . . . . . 31

12 Offer refusal rate among transplanted hearts . . . . . . . . . . . 36

13 Transplant center and donor demographic for transplanted hearts with offer refusal and without any offer refusal . . . . . . . . . . . . . . . . 40

14 Multivariate Cox regression analysis of graft failure . . . . . . . . . . 42

15 Multivariate Cox regression analysis of post-transplant mortality risk 44

16 90-days 1- and 3-Year survival as stratified by number of previous quality-based offer refusals . . . . . . . . . . . . . . 45

17 Zonal potential supply, zonal realized supply, and zonal weight based on the current homogeneous zonal structure . . . . . . . . . . . 63

18 The demographic of the study population . . . . . . . . . . . . . 64

19 Minimum, maximum, median, and average distance traveled by organs (miles) across transplant centers _. . . . . . . . . . . . . 70

20 Access level disparity for heterogeneous zonal structure with different values for maximum circle radius . . . . . . . . . . . . . . . . . . . 73

21 Model validation on different simulation outcomes. . . . . . . . . . . 86

22 Model validation on number of heart transplants based on patients status, age, blood type, and year. . . . . . . . . . . . . 87

23 Model validation on number of deaths based on patients status, age, blood type, and year. . . . . . . . . . . . . . . . . 88

24 Comparison between homogeneous and heterogeneous zonal structures in terms of transplant rate, waition time, mortality rate, and travel distance. . . . . . . . . . . . . . . . .

25 Average of the transplant rates for, computed among the 20 trials of each scenario . . . . . . . . . . . . . . . . . 106 
26 Average number of cycles in the underlying graph for all the scenarios. The average is computed among random graphs, containing 50, 100 and 150 incompatible pairs . . . . . . . . . . . . . . . 108

27 Percentage of matched patients who undergo desensitization for different scenarios . . . . . . . . . . . . . . . . . . . 110

28 Percentage of matched 80-level, 50-level, and 0-level patients who undergo desensitization for different scenarios . . . . . . . . . . . . 111

29 Percentage of matched patients who undergo desensitization for different scenarios . . . . . . . . . . . . . . . . . . . 112

30 Numbers of donor hospitals, transplant centers, incident candidates, and deceased donor kidneys transplanted for each year . . . . . . . 118

31 TSAM Input Data: number of recovered heart organs categorized on some of year, age, race and blood type. . . . . . . . . . . . . . . . 150

32 TSAM Input Data: number of transplant candidates categorized by year, status, age, race, and blood type. . . . . . . . . . . . . . 151

33 The heart allocation system with three status definition for transplant candidates inside the first three zones around donor hospitals . . . . . 152

34 OPTN Data: number of recovered heart organs categorized on some of year, age, race and blood type. . . . . . . . . . . . . . . . . . 154

35 OPTN Data: number of transplant candidates categorized by year, status, age, race, and blood type. . . . . . . . . . . . . . 155 


\section{LIST OF FIGURES}

1 Organ donation and waiting list statistics from 1991 to 2018 (OPTN,

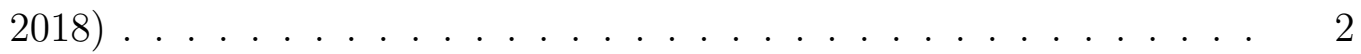

2 Donor Service Area (DSA) of the 58 OPOs in the US . . . . . . . 3

3 UNOS regions . . . . . . . . . . . . . . . . . . . 4

4 The zonal structure around donor hospitals for the heart allocation system . . . . . . . . . . . . . . . . . . . . 4

5 Distribution of the median waiting time across different geographic levels under the 2006 Policy and the 2016 Policy . . . . . . . . . . . . 18

6 Mountain plot for Median Waiting Time . . . . . . . . . . . . 21

7 Median Waiting Time (days) across DSAs for the 2006 Policy and the 2016 Policy .......................... 21

8 Transplant Rate distribution across different geographic levels under the current and the 2016 Policy . . . . . . . . . . . . . . . . . 23

9 Mountain plot for Transplant Rate . . . . . . . . . . . . . . 25

10 Transplant Rate across DSAs under the 2006 Policy and the 2016 Policy 25

11 Pre-Transplant Mortality Rate distribution across different geographic levels for the 2006 Policy and the 2016 Policy . . . . . . . . . . . . . 26

12 Mountain plot for Pre-Transplant Mortality Rate . . . . . . . . . . . 27

13 Pre-Transplant Mortality Rate across DSAs under the 2006 Policy and the 2016 Policy . . . . . . . . . . . . . . . . . . . . . . 28

14 Average Distance distribution across different geographic levels for the 2006 Policy and the 2016 Policy . . . . . . . . . . . . . . . . 29

15 Mountain plot for Average Distance Traveled by Donated Organs . . 31 
16 Average Distance (miles) Traveled by Donated Hearts across DSAs under the 2006 Policy and the 2016 Policy . . . . . . . . . . . . . . . 32

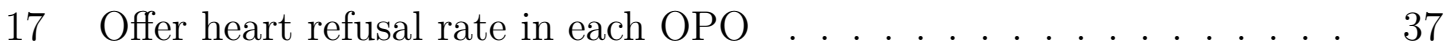

18 Percentage of transplanted vs. discarded organ hearts by year . . . . 38

19 Discarded organ hearts by number of quality-based offer refusals . . . 38

20 Kaplan-Meier analysis demonstrating cumulative survival rate for grafts with no previous quality based offer refusal and grafts with at least one previous quality based offer refusal and $(\log$ rank, $\mathrm{p}<0.001)$. . . . . 46

21 Kaplan-Meier analysis demonstrating cumulative survival rate for grafts stratified by the number of previous quality-based offer refusals (log rank, $\mathrm{p}<0.001) \ldots \ldots \ldots \ldots$

22 Current homogeneous zonal structure composed of six zones around donor hospitals in the heart allocation system. . . . . . . . . . . . . 49

23 Set of four predefined circles around a donor hospital. . . . . . . . . . 52

24 Possible 2 zones drawn from 3 concentric circles around donor hospital j. 54

25 An example for clarifying access level computation for transplant centers. 57

26 An example to illustrate the calculation for potential and realized supply 61

27 Variation in access level across OPOs for homogeneous 2-Zone structure (top), and heterogeneous 2-Zone structure (bottom) . . . . . . . 67

28 Variation in access level across OPOs for homogeneous 3-Zone structure (top), and heterogeneous 3-Zone structure (bottom) . . . . . . . 68

29 Zone A and Zone B radius around donor hospitals for heterogeneous 2-Zone structure . . . . . . . . . . . . . . . . . 69

30 Zone $\mathrm{A}$, Zone $\mathrm{B}$ and Zone $\mathrm{C}$ radius around donor hospitals for heterogeneous 3-Zone structure . . . . . . . . . . . . . . . . . . . . . 69

31 Variation in access level across transplant centers for heterogeneous and homogeneous 2-Zone structures with test (2011-2013) and training data set $(2014) \ldots \ldots \ldots \ldots \ldots \ldots \ldots$ 
32 Variation in access level across transplant centers for heterogeneous and homogeneous 3-Zone structures with test (2011-2013) and training data set (2014). . . . . . . . . . . . . . . . 72

33 Variation in access level across transplant centers for heterogeneous zonal structure ....................... . . 74

34 Variation in travel distance across transplant centers for heterogeneous zonal structure ....................... 75

35 National average distance traveled by organs for heterogeneous zonal structure with different maximum zone radii in comparison with homogeneous zonal structure. The red lines are 2-Zone structures and the blue lines are 3-Zone structures . . . . . . . . . . . . . . . . 76

36 Simulation framework for the heart allocation system in the United

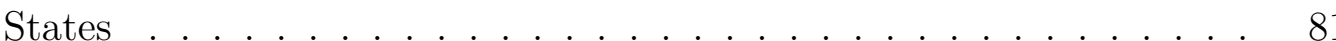

37 Comparison between homogeneous and heterogeneous zonal structures in terms of (a) transplant rate, (b) waiting time, (c) pre-transplant mortality rate, and $(\mathrm{d})$ travel distance. . . . . . . . . . . . . . .

38 Example of a KPD match run representation: (a) compatibility graph; (b) example of a resulting matching of a KPD run without integration with a desensitization protocol; (c) extended compatibility graph; (d) example of a resulting matching of a KPD run integrated with a desensitization protocol . . . . . . . . . . . . . . . 96

39 Modification in the ABO compatibility definition in KPSAM . . . . . 100

40 Adopted Simulation Strategy _. . . . . . . . . . . . . . . . . . . 102

41 Percentage increase in transplant rates for all patients, for patients with different PRA level, and for patients with different blood types with respect to different value of $k$, and when $\delta=2 \%, \delta=6 \%$ and $\delta=11 \%$. 
42 Comparison between the baseline-scenario and the integrated-scenarios of the cumulative percentage of 80-Level patients who are matched in less than a given number of snapshots . . . . . . . . . . . . . . . 109

43 Average percentage of cycles that contains at least one treatment arc for $k \in\{3,4,5,6\}$ and $\delta=2 \%, \delta=6 \%, \delta=11 \% \ldots \ldots 113$

44 Optimized Heterogeneous Circle Size. Each point represents a donor hospital and the color shows the circle size for the donor hospital. . . 120

45 The Variation in Supply/Demand Ratio across Transplant Centers. Each point represents a transplant center. (A) homogeneous circles with size $250 \mathrm{~nm}$, (b) homogeneous circles with size $500 \mathrm{~nm}$, and (c) heterogeneous circles with maximum circle size $500 \mathrm{~nm}$. . . . . . . .

46 Variation in Average Travel Distance across Transplant Centers. The average distance traveled by kidneys for each transplant center under heterogeneous and homogeneous circles. . . . . . . . . . . . . 126

47 Ranging maximum circle size from 200 to $1000 \mathrm{~nm}$, variation in supply/demand ratio across transplant centers under heterogeneous circles on the left side, comparing to the variation in supply/demand ratios under homogeneous circles of size 250 and $500 \mathrm{~nm}$ on the right side. . 126

48 Sensitivity Analysis on Maximum Circle Size. Ranging maximum circle size from 200 to $1000 \mathrm{~nm}$, average distance traveled by donor kidneys under heterogeneous comparing to homogeneous circles. . . . . . . . . 127

49 Sensitivity analysis showing performance of homogeneous circles and heterogeneous circles designed using 2017 data in other years. . . . . 127 


\section{CHAPTER I}

\section{INTRODUCTION}

Organ transplant has an important role in medicine and has become an essential treatment in saving and prolonging lives in a variety of clinical conditions, as well as in increasing life quality (Beyar, 2011). Hearts, kidneys, livers, lungs and pancreata are among the vital organs that are regularly used for transplants.

However, the supply of organ in the United States is just not enough to meet the demand. In the U.S. nearly 50,000 people per year either die on the transplant waiting list or are removed from the list after waiting so long that they have become too sick to undergo transplant surgery (OPTN, 2018), and the waiting list continues to grow, where currently more than 100,000 people are waiting for transplants(OPTN (2018); see Figure 1).

This shortage of supply has led to the design of different policies for a better and more efficient allocation of the available organs to people.

Organ allocation in the United States is administrated by the United Network of Organ Sharing (UNOS), which is the only administrative entity contracted by the Department of Health and Human Services (DHHS) to administer the Organ Procurement and Transplantation Network (OPTN). OPTN was created in 1984 by the National Organ Transplant Act (NOTA) with the aim of creating an equitable organ allocation policy. To allocate organs, UNOS currently uses a multi-stage process in which the U.S. is divided into 58 Organ Procurement Organizations (OPOs) (Figure 2), which are grouped into 11 UNOS regions (Figure 3). Each of these OPOs is responsible for acquiring and distributing of the organs procured within its service area, known as the Donor Service Area (DSA). 


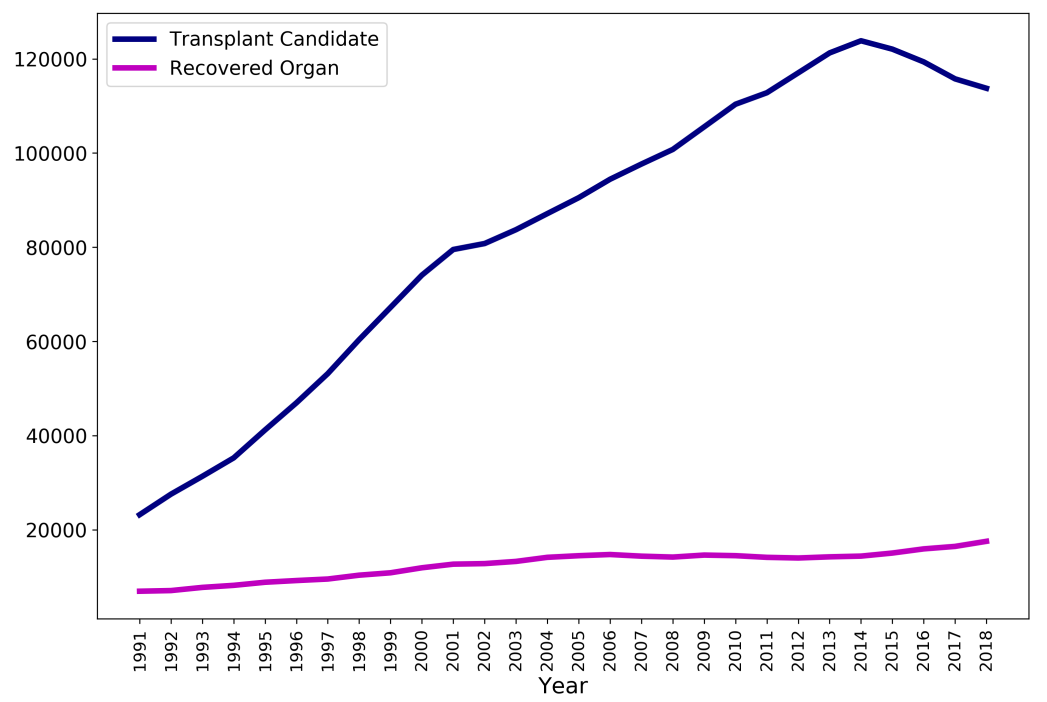

Figure 1. Organ donation and waiting list statistics from 1991 to 2018 (OPTN, 2018)

Allocation rules depend on specific criteria which are different from organ to organ. These criteria are developed and updated based on discussions involving a combination of clinical scientific data and ethics based on clinical scientific data. Even if the overall system is complex, it is possible to make some generalizations. Generally, before an organ is allocated, all transplant candidates on the waiting list who are incompatible with the donor are automatically eliminated from any potential match, and then a possible match is sought among compatible candidates. Looking at the system from a very high level, compatible transplant candidates are prioritized based on two main factors: (1) the medical condition of the patient (including how urgently, she/he needs the organ and the survival benefits), and (2) the distance between the hospital where the organ became available (i.e., donor hospital) and the transplant center where the candidate is registered. Both factors influence the success of a transplant and the post transplant survival of the patient. Distance, in particular, highly influences the quality of the organ to be transplanted. Indeed, every organ can be transplanted within a maximum specified cold ischemia 


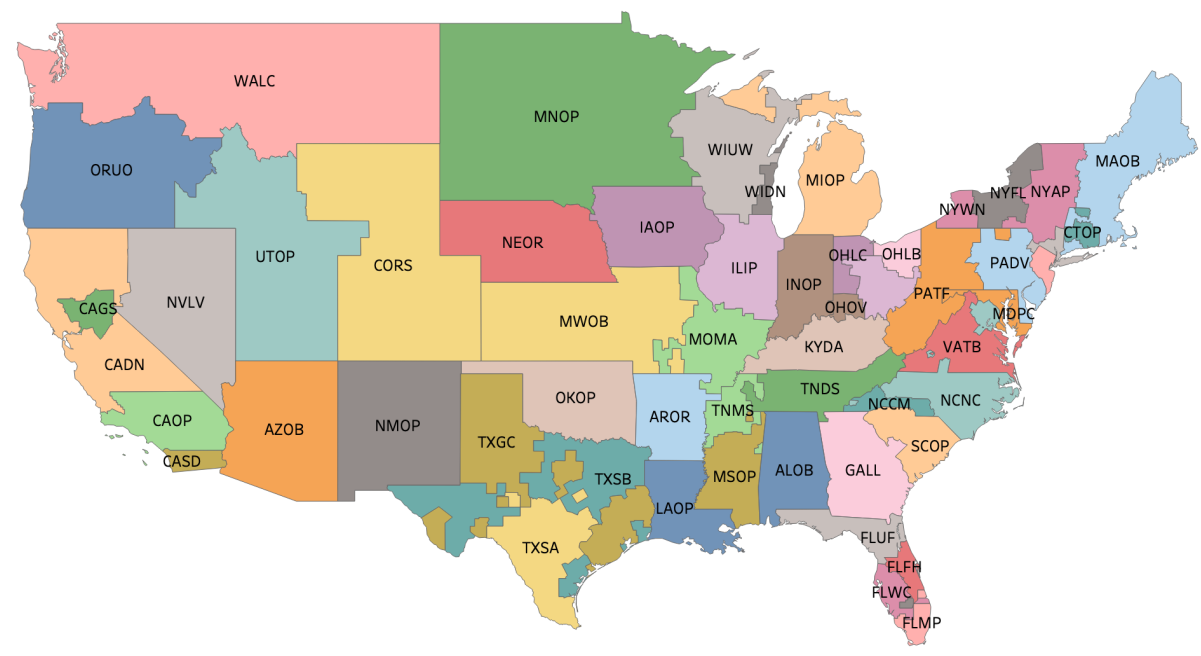

Figure 2. Donor Service Area (DSA) of the 58 OPOs in the US

time, which is the time between organ-donor retrieval and the transplantation. The shorter the cold ischemia time, the better the quality of the organ.

The current system distributes the available organs near donor hospitals first and then looks for compatible patients at a further distance with exceptions for patients with high medical urgency (OPTN-Policy, 2018).

For example, the kidney allocation system uses DSAs and UNOS regions for allocating the organs. When a kidney is donated within a specific DSA, patients registered in that DSA are prioritized to receive the kidney, and, if any match for the kidney is not found in that DSA, the allocation rule expands the search to the region where the kidney is donated. In the end, if no kidney transplant recipient has been located in the region, the search for a match expands nationally.

As another example, the allocation system for heart transplants is based on the urgency of patients and concentric zonal structure around donor hospitals (Figure 4). UNOS assigns a status to each transplant candidate based on their medical condition (status 1-6, with 1 being the most severe and 6 being the least severe). A guiding principle for heart allocation is to prioritize those in greatest need within an acceptable geographic distance. The typical set of zones is defined 


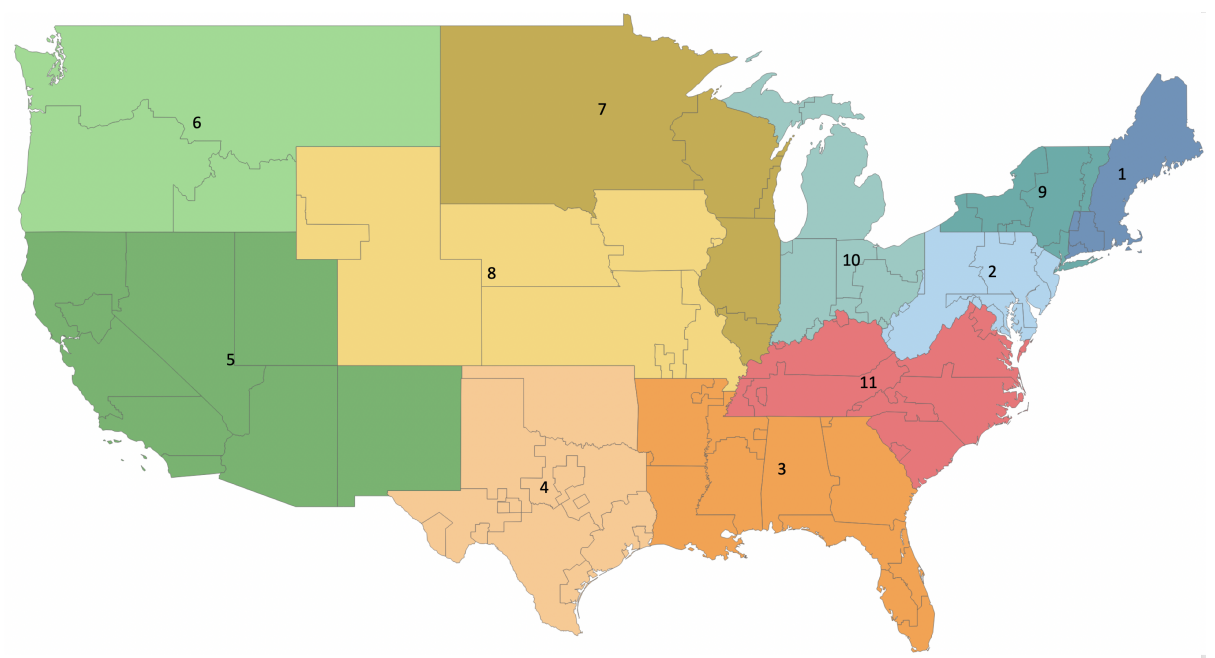

Figure 3. UNOS regions

through concentric circles (see Figure 22) with increasing radii of 250 miles (Local), 500 miles (Zone A), 1,000 miles (Zone B), 1,500 miles (Zone C), 2,500 miles (Zone D), and outside 2,500 miles (Zone E). Thus, transplant candidates registered at the transplant centers located within the donor hospital's local area receive offers first, followed by the candidates at the transplant centers in the successive zones.

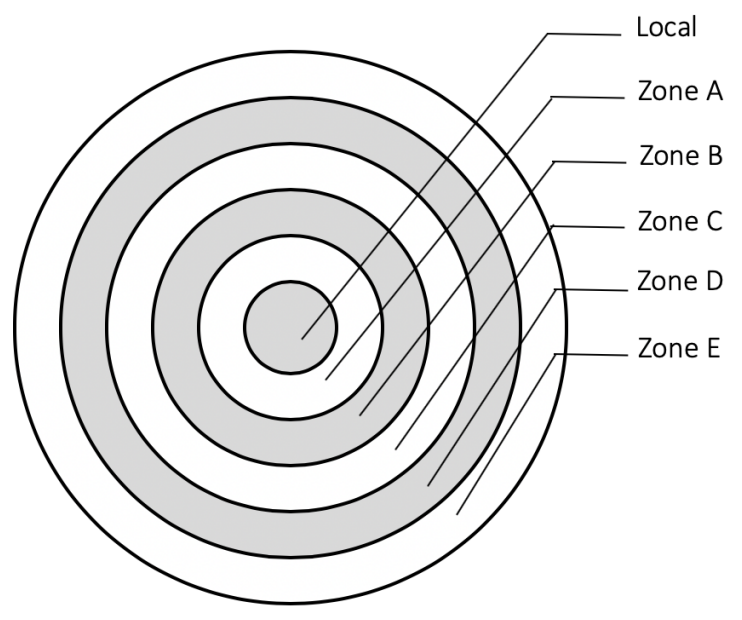

Figure 4. The zonal structure around donor hospitals for the heart allocation system 


\section{A The Big Challenge: Geographic Disparity in Access to Transplants}

All the allocation policies which are currently applied in the U.S. have been developed and changed over the years to comply with the principles stated in the regulation known as the "Final Rule", which was issued by DHHS in 1998 to ensure an equitable allocation system (Snyder et al., 2018). However, the current allocation system can still lead to very unfair cases. Existing studies on organ transplants report various disparities in access to and outcomes of transplantation. Disparities have been found in terms of race, socioeconomic status, insurance type, and location of candidate's residency. Disparity in access to transplant due to candidates' locations , also known as "geographic disparity", is the first and foremost discussed. Geographic disparity in access to transplant occurs, for example, when an organ may go to a patient who has a less severe status than another candidate, who is farther away from the procuring $\mathrm{OPO}$, contradicting one of the principals of the final rule, according to which an equitable allocation of donor organs for potential recipients "shall not be based on the candidate's place of residence or place of listing" (DHHS, 1999). ${ }^{1}$

Transplant researchers reported geographic disparitys in access to transplant across transplant centers, OPOs and regions in terms of transplant rate, waiting time, pre-transplant survival rate, to mention some of the metrics which are used to compute access to transplant.

\footnotetext{
${ }^{1}$ The urgency and importance of such a drawback of the current system is evidenced by a recent change in the U.S. lung allocation protocol which was triggered after a lawsuit was initiated due to a severe disparity in access to a lung in the border region of New Jersey and New York (Snyder et al., 2018). Specifically, on November 19th, 2017, 21 years old Miriam Holman, who had been waiting for a donor lung in a hospital in New York City, filed a temporary restraining order against the DHHS to allocate donor lungs based on medical priority instead of candidate place of residence. At the time of the lawsuit, lungs were allocated first to the candidates within a geographic DSA, then to other candidates in transplant centers further away. Since the geographic territories of the DSA are arbitrary, it was possible that a candidate registered in Jersey City, NJ with a medical priority less than Miriam's receives an available lung before she does. The US District Court ordered the DHHS to immediately review the lung allocation policy in light of the Final Rule requirements. In less than a week, organ allocation for donor lungs was changed, considering as a first level distribution not the DSA rather a 250 nautical miles circle around donor hospitals.
} 
A recent study on the heart allocation system, for example, shows that the transplant rate performed on higher urgency candidates varies significantly from $37 \%$ to $86 \%$ across UNOS regions (Meyer et al., 2015). Another study of kidney allocation indicates that the waiting time for transplantation ranges from 6 months to more than 5 years across DSAs (Davis et al., 2014). In the liver allocation system, the transplant rate and pre-transplant death rate varied 20-fold and 3.3-fold, respectively, across DSAs (Yeh et al., 2011).

In light of this, several researchers have addressed the problem of reducing geographic disparity in access to transplant for different organs. The approaches can be grouped into two main categories:

1. Better Usage of Donated Organs: One of the main causes of geographic disparity is the uneven distribution of supply and demand across the country. The gap between supply and demand becomes even larger because of the variability in organ usage rate, organ waste and other factors which influence the effective use of donated organs (Mathur et al., 2010). A better utilization of donated organs would be beneficial in reducing the gap between supply and demand.

2. Alternative Allocation Policies: Several studies propose changes in the current allocation policies. Gentry et al. (2013), for example, proposed changing the number of UNOS regions from 11 to 8 to reduce geographic disparity in access to liver transplants. Davis et al. (2015) studied possible sharing strategies among OPOs to reduce geographic disparity in access to kidney transplants.

Despite the existing studies to address the problem, there is a consensus that more research is needed in this area (Koizumi, 2010). In this context, our research focuses on new methods to reduce geographic disparity in access to kidney and heart transplants. The proposed methods fall into two research categories, mentioned before. Specifically, to improve access to heart transplants we design a 
novel robust optimization model to modify the current heart allocation system and evaluate the performance of our optimization model using a simulation model, which we develped (Chapter II - IV). To increase access to kidney transplants, we propose an optimization-simulation approach for a better utilization of donated organs from living donors within a Kidney Pair Donor (KPD) ${ }^{2}$ program (Chapter V). Additionally, to reduce geographic disparity in access to kidney transplants, we used the optimization model developed in Chapter III, to design geographic boundaries for the U.S. kidney allocation system. The structure of this dissertation and the contributions of the research are briefly outlined next.

Chapter II: Analyzing Access to Heart Transplants In this chapter we assessed the current level of access to heart transplants in the U.S. and the current level of usage of donated hearts. The chapter is divided into two parts:

In the first part of the chapter we investigated the impact of recently approved heart allocation policy on the geographic disparity in access to heart transplants (Policy, 2016). The new policy was introduced with the aim of reducing waitlist mortality and improving post-transplant survival. The transplant community has voiced a number of concerns regarding a possible increase in geographic disparity in access to transplants. No study in the literature addresses such a concern, which is instead the focus of our analysis. Specifically, we used the Thoracic Simulation Allocation Model (TSAM) ${ }^{3}$ to systematically compare the previous policy and the new implemented policy. We compared four performance indicators: waiting time, transplant rate, pre-transplant mortality rate, and average distance traveled by donated hearts.

In the second part of chapter we analyzed the current level of usage of

\footnotetext{
${ }^{2}$ KPD program allows incompatible donor-patient pairs to exchange their living donors with other incompatible pairs within the program (Montgomery et al., 2011; Roth et al., 2004)

${ }^{3}$ TSAM is a simulation software developed by the US Scientific Registry of Transplant Recipients (SRTR) (TSAM, 2017). TSAM has the ability to simulate the allocation of hearts to candidates waiting for heart transplants based on an event-sequenced Monte Carlo technique.
} 
donated hearts by exploring data on discarded hearts from 2003 to 2015 . We examined the percentage of donor hearts offer refusal in each OPO, to quantify geographic disparity in donor heart offer refusal across OPOs. We performed a post-transplant survival analysis to assess the transplant outcome of the hearts with previous offer refusals. The results from this analysis can help policy makers in improving the criteria used to discard donated organs, in identifying the OPOs with higher offer heart refusal rate, and in improving the OPOs' performance.

\section{Chapter III: An optimization approach to Address Geographic Disparity} in Access to Heart Transplant The current zonal structure (Figure 4) used in heart allocation system is homogeneous across donor hospitals and does not consider the heterogeneity in supply and demand. In Chapter III, we designed a novel mathematical optimization model to minimize geographic disparity in access to heart transplants by designing a heterogeneous zonal structure around donor hospitals. With the heterogeneous zonal structure the zone radius for different donor hospitals can be different based on the distribution of supply and demand.

\section{Chapter IV: Simulation Model for Evaluating Heart Allocation Policy}

We have developed clinically detailed simulation model for the U.S. heart allocation system to assess and compare the impact of alternative allocation policies, which was not feasible with the TSAM. For this purpose, we have independently replicated most of the concepts framed in TSAM and we validated our model with historical data on the U.S. heart transplants. We used this model to evaluate the effect of heterogeneous zonal structure, developed in Chapter III, on geographic disparity in access to heart transplants in the United States.

\section{Chapter V: Optimal Integration of Desensitization Protocols into Kidney Paired Donation (KPD) Programs We designed and implemented an optimization-simulation approach to analyze the integration of desensitization}


therapy for patients in a KPD program. The optimization-simulation approach simulates the dynamic and the evolving environment of a KPD program overtime. We used our approach to measure the benefit of such integration by performing an extensive experimental analysis which compares the output of a KPD program under different scenarios.

The idea of systematically integrating desensitization therapy in a KPD program is highly innovative. All the current methodological approaches do not consider such an option, which is instead receiving increasing more attention as a promising intervention performed in a KPD framework (Montgomery et al., 2005; Montgomery, 2010; Montgomery et al., 2011).

\section{Chapter VI: Allocating Kidney Through Heterogeneous Circles The}

OPTN recently approved a plan to eliminate DSAs and region from kidney allocation system and to allocate kidneys in 250 miles fixed-distance circles around donor hospitals, with a continuous priority boost for candidates nearer to the donor hospital. Homogeneous circles, using the same size circle for every donor hospital, might not substantially reduce geographic disparity because of heterogeneity in supply and demand. We designed heterogeneous circles around donor hospitals to reduce the variation of supply/demand ratios across transplant centers using an integer programming model. 


\section{CHAPTER II}

\section{ANALYZING ACCESS TO HEART TRANSPLANTS}

\section{A Analyzing Geographic Disparity in Access to Heart Transplants}

\section{Introduction}

Heart failure is a growing health problem affecting over 5 million adults in the United States (Go et al., 2013b). Heart transplantation is the definitive therapy with the best favorable outcomes for end-stage (i.e., Stage D) heart disease patients: 1-year survival rate for adult patients who underwent a heart transplant from 2007 through 2009 was $88 \%$, while 3-year and 5-year survival rates were $81 \%$ and $75 \%$, respectively (Israni et al., 2014b). Without a transplant, the 5-year survival rate is only about 20\% (AFZAL AMMAR et al., 2007).

Heart allocation system, like any other organ, is administrated by UNOS. UNOS's mission is to ensure fair and equitable allocation of organs as stated in the Code of Federal Regulations, which reads "neither place of residence nor place of listing shall be a major determinant of access to a transplant" (DHHS, 1999). UNOS/OPTN established heart allocation policy in 1988. The system remains in place today, with only two major policy changes since its inception (Meyer et al., 2015). The previous allocation system in heart transplant (referred to as the 2006 Policy in the rest of the chapter) is based on the urgency of patients and concentric geographical zones around donor hospitals. UNOS assigns all transplant candidates a status which is based on their medical condition, namely Status 1A, 1B, and 2, with Status 1A being the most urgent patients. A guiding principle for heart allocation is to give highest priority to those in greatest need within an acceptable 
geographic distance. The typical geographic sequence for heart allocation is local (i.e., DSA) first, followed by increasing concentric circles, specifically of radii equal to 500 miles (Zone A), 1000 miles (Zone B), 1500 miles (Zone C), 2500 miles (Zone D), and more than 2500 miles (Zone E). Waitlisted transplant candidates who are registered at the transplant centers located within the DSA receive offers first, followed by the candidates at the transplant centers within the next concentric circle area(Table 1 shows the start part of allocation sequence for 2006 Policy).

The OPTN/UNOS Thoracic Organ Transplantation Committee pointed out that despite all the improvements resulting from the 2006 Policy, mortality on waiting lists is still high for certain groups of patients (Meyer et al., 2015). As a result, in December 2016 the thoracic committee approved a new policy (referred to as 2016 Policy in the rest of the chapter) (OPTN, 2016). The proposed policy had the twofold aim of (i) reducing waiting list mortality rates among the most severe patients, and (ii) improving post-transplant survival rates. This new allocation system groups recipients into 6 severity statuses (Status 1 to 6 , status 1 corresponding to the most severe patient) in contrast with the previous three levels. Table 2 shows the correspondence between the six-tiered severity status system approved in 2016 and the three-tiered severity status classification of the 2006 Policy.

The 2016 Policy introduces the so-called "broader sharing" which alters the sequence of allocation for the most urgent candidates. Under the broader sharing, hearts from adult deceased donors will be offered first to compatible adult status 1 within the local DSA plus Zone A around the donor hospital, then to compatible adult status 1 candidates within Zone B. If no matches are made for these candidates, hearts will be offered to the candidates in lower urgency statuses beginning at the local DSA (Table 3 shows the start part of allocation sequence for 2006 Policy) (OPTN, 2005).

Prior research reports that access to a heart transplant, with 2006 policy, 
depends on the location due to the geographic variation in the donor-patient ratio (Kobashigawa, 2014; Meyer et al., 2015; Rivard et al., 2018) and that more research is warranted in this area (Kobashigawa, 2014). A study demonstrates that the percentage of transplants performed on higher urgency adult candidates (Status 1) varies from $37 \%$ to $86 \%$ across geographic regions (Meyer et al., 2015). This is contrary to the founding principles of UNOS, which focus on recipient chance wait list equality.

While the 2016 Policy is expected to improve transplant rates and waiting times for the most severe patients, the transplant community has voiced a number of concerns regarding geographic disparity in access to heart transplant in this new approved policy (OPTN-Policy, 2018). The main goal of this chapter is to analyze how access to heart transplant varies across geography both under the 2006 and the 2016 Policy using simulations. To achieve this goal, we used a heart version of a widely used simulation software known as the Simulated Allocation Model (SAM), namely the Thoracic Simulated Allocation Model (TSAM). SAM is developed by the US Scientific Registry of Transplant Recipients (SRTR) (SAM, 2017) to examine the impacts of different organ allocation rules. Prior studies have used SAM extensively, especially for testing different liver allocation protocols (Shechter et al., 2005; Wiesner et al., 2001; Harper et al., 2000; Schaubel et al., 2009; Roberts et al., 2006; Freeman Jr et al., 2004; Gentry et al., 2013). The application of TSAM to test different heart allocation protocols is limited to evaluating whether under the 2016 Policy there is an improvement on transplant rates and waiting times for the most severe patients (Israni et al., 2014b). Implications of the 2016 Policy on geographic disparity have not been evaluated. Such an analysis is warranted since alleviating geographic disparity is one of the stated objectives of the 2016 Policy. This is the focus of this research. The TSAM software allowed us to examine disparities across different geographic levels in the United States (US), namely across the 111 heart transplant centers (TC) in the US, across the 58 DSA and 
across the 11 UNOS regions.

TABLE 1

Adult heart allocation (2005 to present) (Meyer, 2015)

\begin{tabular}{ll}
\hline Location & Patient Status \\
\hline DSA & Status 1A \\
DSA & Status 1B \\
Zone A & Status 1A \\
Zone A & Status 1B \\
DSA & Status 2 \\
Zone B & Status 1A \\
Zone B & Status 1B \\
Zone A & Status 2 \\
Zone B & Status 2 \\
\hline
\end{tabular}

TABLE 2

Patient status classification for from 1998 to present and approved in 2016

\begin{tabular}{ll}
\hline 2006 Policy & 2016 Policy \\
\hline Status 1A & Status 1 \\
& Status 2 \\
& Status 3 \\
\hline Status 1B, with a small & Status 4 \\
group of status 2 & \\
\hline Status 2 & Status 5 \\
& Status 6 \\
\hline
\end{tabular}




\section{TABLE 3}

Heart allocation scheme for adult candidates approved in 2016 (2016 Policy)

\begin{tabular}{ll}
\hline Zone & Status \\
\hline DSA+Zone A & Status 1 \\
Zone B & Status 1 \\
DSA+Zone A & Status 2 \\
Zone B & Status 2 \\
DSA & Status 3 \\
DSA & Status 4 \\
Zone A & Status 3 \\
DSA & Status 5 \\
Zone B & Status 3 \\
DSA & Status 6 \\
Zone C & Status 1 \\
Zone C & Status 2 \\
Zone C & Status 3 \\
Zone A & Status 4 \\
Zone A & Status 5 \\
Zone A & Status 6 \\
\hline
\end{tabular}

\section{Methods and Materials}

\subsection{Thoracic Simulation Allocation Model}

In this study the Thoracic Simulation Allocation Model (TSAM) was used to quantify access to heart transplants in the US. TSAM is a simulation software developed by the US Scientific Registry of Transplant Recipients (SRTR) (SAM, 2017). TSAM has the ability to simulate the allocation of hearts to candidates waiting for heart transplants based on an event-sequenced Monte Carlo technique. 
TSAM has been purposefully designed to serve studies investigating alternative organ allocation models (Parker et al., 2017). It can use a variety of allocation rules to determine how a series of thoracic organs would be allocated to a list of potential recipients under each of the rules considered. The main process of TSAM is to receive organ arrivals, use the 2014 allocation model rule and match the organ with potential patients, offer the organ to them and grant the organ to the one who accepts it. Meanwhile, it tallies patients on the waitlist, deaths on the waitlist, and discarded organs. The simulation involves two random components to reflect (i) the uncertainty in a patient's decision to accept or reject an organ, and (ii) to capture the uncertainty in the life expectancy of a patient after receiving an organ. TSAM has its own formulas to evaluate these random components. The built-in input data covers the two-year study period from July 1, 2009 to July 1, 2011, which implements the allocation policy as of April 2014. The input files contain patient arrival, organ arrival, patient waiting list, allocation rule, ABO blood compatibilities, patient status updates and a few more types of data. Each file has an extensive amount of medical information for patients which are used as factors in determining who qualifies for a transplant. After each run, TSAM provides a series of output files which contain information on graft recipients, discarded organs, patient death on waitlist, final patient waitlist and a few more types of information. Patients at least 18 years old were considered in the analysis, with a total of 8,593 candidates. Of these patients, 2,621 candidates were registered at the beginning of the study period, and 5,972 new candidates arrived during the study period. Among the patients there were 159 requiring combined heart-lung transplant who were excluded from the study. The final dataset included a total of 8,434 candidates who were registered for a diseased donor heart transplant and 3,770 transplant recipients.

TSAM was used to simulate the two allocation scenarios described in Table 1 and Table 3, that is:

1. 2006 Policy: three-tiered patient urgency status and allocation policy as in 
Table 1.

2. 2016 Policy: six-tiered patient urgency status with modified broader sharing scheme approved in 2016, as in Table 3.

\subsection{Statistical Analysis}

For each allocation scenario, the following performance measures were computed for three different geographic levels, namely TC, DSA, and UNOS regions:

1. Median Waiting Time to Transplantation: Median total waiting time among all the candidates who received a heart for each geographic level considered.

2. Transplant Rate: Ratio between the number of heart transplants performed and the number of candidates on the waitlist for each geographic level considered.

3. Pre-Transplant Mortality Rate: Ratio between the number of heart transplant candidates who died while on the transplant waitlist and the total number of candidates on the waiting list for each geographic level considered.

4. Average Distance Traveled by Donated Hearts: Average distance traveled by donated hearts during the study period for each geographic level considered.

The first three are commonly used measures to evaluate the equity aspect of an organ transplant system (Israni et al., 2014b; Kobashigawa, 2014; Meyer et al., 2015), while the last measure has a clinical importance since a longer organ transfer distance is associated with a longer cold ischemia time, which in turn leads to worse transplant outcomes especially for heart transplants.

To quantify geographic disparity for each performance measure, several disparity metrics, including Standard Deviation (SD), Mean Square Difference from the Best Rate (MSBR), Range, and Interquartile Range (IQR) were considered. To 
evaluate whether the geographic disparity among different geographic levels and under the two policies is statistically significant, the non-parametric Kruskal-Wallis test was applied to compare the medians of the measures at the different geographic levels.

\section{Results}

\subsection{Median Waiting Time to Transplantation}

The median waiting time (number of days) is calculated among all the patients at each geographic level, that is, for each TC, for each DSA, and for each region. For instance, the median waiting time at each TC (DSA, region) is the median of the waiting times of all the patients that had a heart transplant in that TC (DSA, region). Figure 1 shows the boxplots for the distribution of the median waiting time for each geographic level for both policies. For example, at the TC level the boxplot represents the distribution of the 111 median waiting times each computed in one TC in the US. Similarly, at the DSA level the boxplot represents the distribution of the 58 median waiting times each associated with one of the DSA in the US. From the boxplots in Figure 5 it can be observed that at the TC level, the median waiting time varies from 27 to 986 days under the 2006 Policy, and from 9 to 933 days under the 2016 Policy. The median waiting time across DSAs under the 2006 Policy ranges between 27 and 264 days, and between 14 and 156 days under the 2016 Policy. Across regions, the median waiting time ranges between 88 and 256 days under the 2006 Policy, and between 39 and 149 days under the 2016 Policy. Table 4 shows the median and average value of the distribution of the median waiting time at different geographic level. This table shows an improvement in the median waiting time under the 2016 Policy for each geographic level. 


\section{TABLE 4}

Median Waiting Time (days): average and median values at the TC, DSAs and the region level for different urgency statuses while on the waiting list

\begin{tabular}{lll|ll}
\hline \multicolumn{3}{c|}{ 2006 Policy } & \multicolumn{2}{l}{ 2016 Policy } \\
\hline & Average & Median & Average & Median \\
\hline Region & & & & \\
All & 150 & 143 & 92 & 91 \\
Status 1A & 39 & 31 & 13 & 7 \\
Status 1B & 56 & 50 & 36 & 40 \\
Status 2 & 150 & 143 & 0 & 0 \\
DSA & & & & \\
All & 145 & 144 & 89 & 88 \\
Status 1A & 35 & 30 & 11 & 8 \\
Status 1B & 61 & 58 & 37 & 34 \\
Status 2 & 3 & 0 & 2 & 0 \\
TC & & & & \\
All & 171 & 151 & 110 & 92 \\
Status 1A & 42 & 34 & 13 & 10 \\
Status 1B & 71 & 60 & 48 & 34 \\
Status 2 & 17 & 0 & 17 & 0 \\
\hline
\end{tabular}

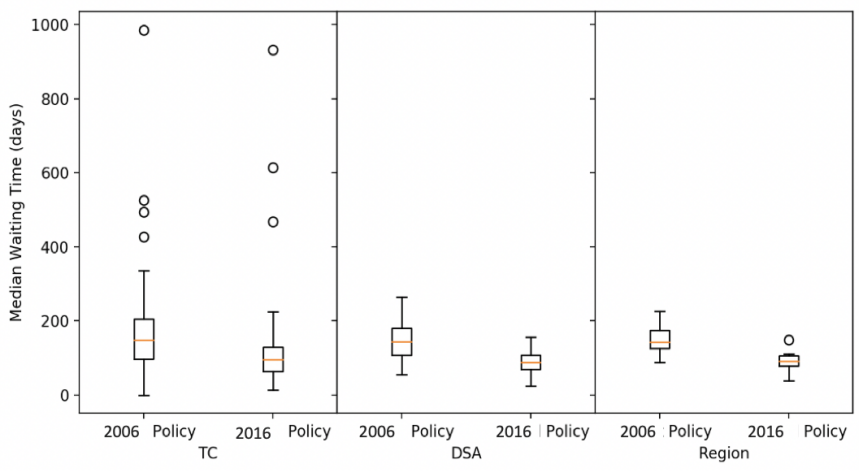

Figure 5. Distribution of the median waiting time across different geographic levels under the 2006 Policy and the 2016 Policy

A quantification of the geographic disparity in the median waiting time across the different geographic levels can be assessed looking at the results in Table 
5, Figure 6 and Figure 7. Table 5 shows our disparity metrics for the median waiting time at the TC, DSA, and region levels for different urgency statuses. The higher the value the higher the geographic disparity. Values in the table show that both policies suffer from geographic disparity in the median waiting time, however, geographic disparity is lower under the 2016 Policy. The non-parametric

Kruskal-Wallis test applied to test the null hypothesis of equality of medians at a given geographic level (i.e., $H_{0}: m_{1}=m_{2}=\cdots=m_{k}$, where $m_{i}$ is the median of group $i$, and $k$ is the total number of groups in the given level), and under the both policies. The test revealed that the medians are statistically significantly different at all levels and under both policies (at the TC level: 2006 Policy: $H=283.1$, $P<0.001,2016$ Policy: $H=421.78, P<0.001$; at the DSA level: 2006 Policy: $H=150.75, P<0.001,2016$ Policy: $H=251.48, P<0.001$; and at the region level: 2006 Policy: $H=74.16, P<0.001,2016$ Policy: $H=141.31, P<0.001)$.

Figure 6 displays the mountain plot of the median waiting time under the two allocation policies across DSAs. The graph shows the cumulative distribution of the median waiting time across DSAs peaked at the median. The increasing part of the plot displays the data up to $50^{\text {th }}$ percentile and the decreasing part of the plot displays the data from the $50^{\text {th }}$ to $100^{\text {th }}$ percentile. A wider mountain denotes greater disparity of the plotted measure at the DSA level. Using this plot we can simultaneously evaluate whether the median value of the considered measure has changed under the two policies (if the two plots are shifted with respect to each other), and whether the measure shows geographic disparities at the DSA level (a wider plot reveals greater disparity). It can be observed that, as already revealed by the results in Figure 5 and Tables 4-5, under the 2016 Policy the median waiting time and the geographic disparity in the median waiting time across DSAs decrease. Finally, Figure 7 allows for a visualization of the geographic disparity across DSAs, as it shows the map of the median waiting time in different DSAs under the 2006 Policy (on the left) and under the 2016 Policy (on the right). Longer median 


\section{TABLE 5}

Median Waiting Time: disparity metrics at the TC level, DSA level and region level for different urgency statuses while on the waiting list

\begin{tabular}{lllll|llll}
\hline \multicolumn{5}{c}{ 2006 Policy } & \multicolumn{3}{l}{ 2016 Policy } \\
\hline Region & SD & MSBR & Range & IQR & SD & MSBR & Range & IQR \\
All & 40 & 73 & 138 & 78 & 28 & 59 & 109 & 39 \\
Status 1A & 24 & 32 & 77 & 43 & 11 & 14 & 39 & 15 \\
Status 1B & 25 & 54 & 89 & 44 & 20 & 39 & 62 & 41 \\
Status 2 & 0 & 0 & 0 & 0 & 0 & 0 & 0 & 0 \\
DSA & & & & & & & & \\
All & 52 & 129 & 237 & 98 & 33 & 81 & 141 & 57 \\
Status 1A & 28 & 45 & 110 & 56 & 10 & 15 & 44 & 18 \\
Status 1B & 38 & 72 & 208 & 64 & 25 & 45 & 119 & 47 \\
Status 2 & 15 & 15 & 106 & 0 & 7 & 7 & 42 & 0 \\
TC & & & & & & & & \\
All & 119 & 187 & 959 & 135 & 110 & 149 & 924 & 84 \\
Status 1A & 34 & 54 & 160 & 54 & 12 & 18 & 54 & 20 \\
Status 1B & 65 & 96 & 295 & 94 & 64 & 80 & 558 & 75 \\
Status 2 & 95 & 96 & 896 & 4 & 96 & 97 & 896 & 2 \\
\hline
\end{tabular}

waiting times are represented by darker shades, additionally, a more uniform map corresponds to less geographic disparity. The map for the 2016 Policy looks lighter that the map under the 2006 Policy, showing an overall decrease in the median waiting time. However, both the policies show the same spectrum of shades, revealing, for example, that the median waiting time is higher in the northern areas than in the eastern areas of the US. That is, geographic disparity exists under both the policies and is affecting the same areas. 


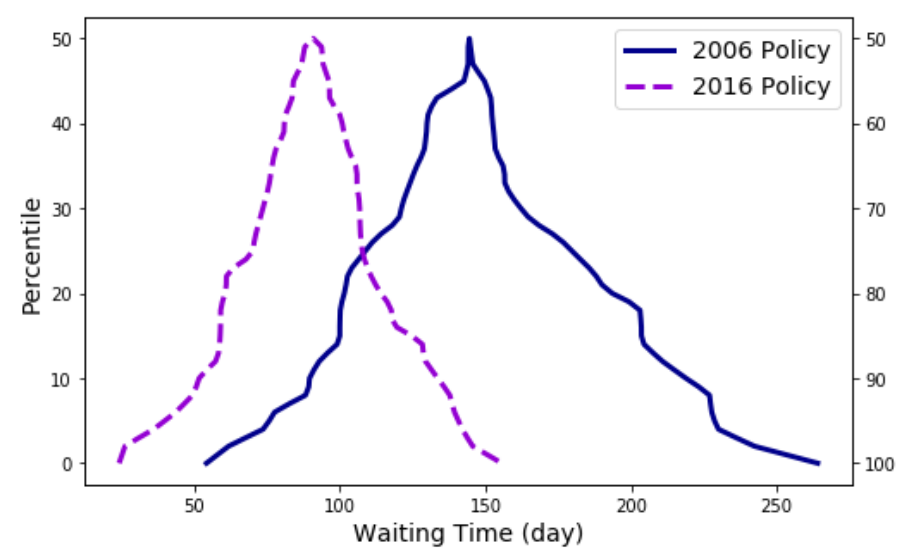

Figure 6. Mountain plot for Median Waiting Time
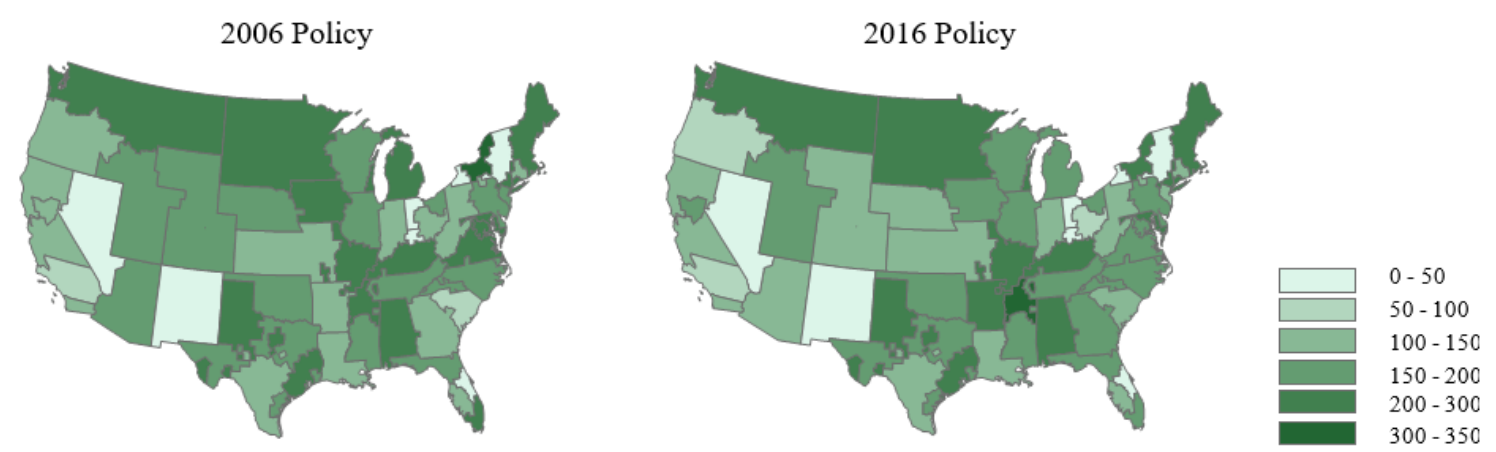

Figure 7. Median Waiting Time (days) across DSAs for the 2006 Policy and the 2016 Policy

\subsection{Transplant Rate}

Transplant rate in each geographic level is computed as the ratio between the number of patients who received a transplant and the total number of patients waiting for a heart transplant at that geographic level. Figure 8 shows the boxplots of the distribution of the transplant rate for each geographic level under both policies. The figure shows that at the TC level, transplant rate ranges between 0.04 and 0.52 under the 2006 Policy, and between 0.24 to 1 under the 2016 Policy. The transplant rate across DSAs under the 2006 Policy ranges between 0.12 and 0.50 , 
and between 0.31 and 0.67 under the 2016 Policy. The transplant rate across regions ranges between 0.18 to 0.34 under the 2006 Policy, and between 0.36 and 0.57 under the 2016 Policy. Table 6 shows the median and the average of the transplant rates for different geographic levels. Results in the table reveal that under the 2016 Policy the median and average transplant rate improve for all geographic levels. Table 7 summarizes the disparity metrics for transplant rates at the TC, DSA, and region levels for different urgency statuses of the patients while on the waiting list. Each metric reveals that, under the 2016 Policy, geographic disparity in transplant rate increases for each status and for each geographic level. Results from the non-parametric Kruskal-Wallis test indicate that the median values of the transplant rates at the DSA level are statistically significantly different for both policies. (2006 Policy: $H=72.53, P=0.02,2016$ Policy: $H=66.75, P=0.04)$. On the other hand, the median values across regions are statistically significantly different under the 2006 Policy $(H=22.02, P=0.015)$, while they are not under the 2016 Policy $(H=17.07, P=0.07)$. Figure 9 shows the mountain plot of the distribution of the transplant rate across DSAs under the current and the 2016 Policy. The graph shows, that transplant rates improve under the 2016 Policy; however, the mountain plot under the 2016 Policy is wider than the mountain plot under the 2006 Policy, revealing an increase in geographic disparity in transplant rate at the DSA level. 


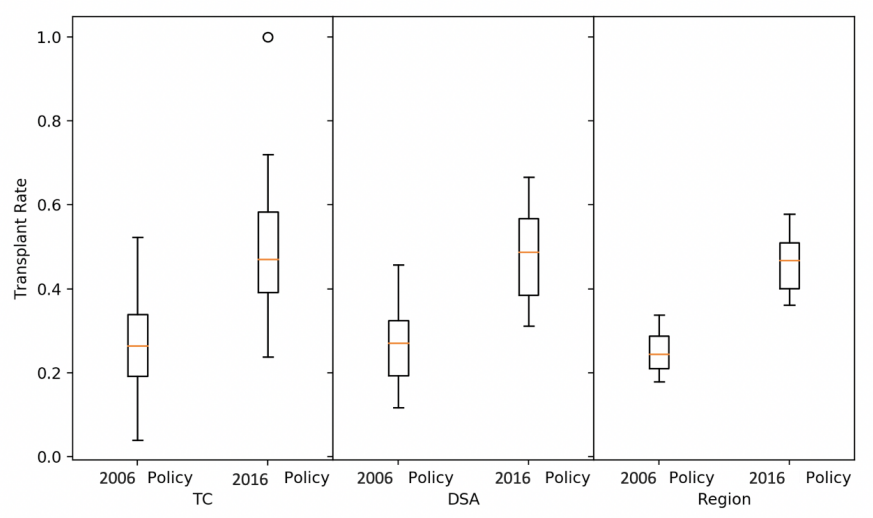

Figure 8. Transplant Rate distribution across different geographic levels under the current and the 2016 Policy

\section{TABLE 6}

Transplant Rate: average and median values at the TC, DSA and region level for different urgency statuses while on the waiting list

\begin{tabular}{lll|ll}
\hline \multicolumn{3}{c|}{ 2006 Policy } & \multicolumn{2}{l}{ 2016 Policy } \\
\hline Region & Average & Median & Average & Median \\
All & 0.25 & 0.25 & 0.46 & 0.47 \\
Status 1A & 0.61 & 0.64 & 0.82 & 0.82 \\
Status 1B & 0.19 & 0.19 & 0.52 & 0.56 \\
Status 2 & 0.02 & 0.01 & 0.08 & 0.05 \\
$D S A$ & & & & \\
All & 0.27 & 0.27 & 0.49 & 0.49 \\
Status 1A & 0.62 & 0.64 & 0.83 & 0.84 \\
Status 1B & 0.23 & 0.19 & 0.57 & 0.58 \\
Status 2 & 0.02 & 0.00 & 0.09 & 0.04 \\
TC & & & & \\
All & 0.27 & 0.27 & 0.49 & 0.47 \\
Status 1A & 0.62 & 0.64 & 0.80 & 0.85 \\
Status 1B & 0.22 & 0.18 & 0.54 & 0.57 \\
Status 2 & 0.02 & 0.00 & 0.08 & 0.03 \\
\hline
\end{tabular}

Figure 10 shows the choropleth map of the transplant rate across DSAs under the 2006 Policy (on the left) and the 2016 Policy (on the right). Higher transplant 


\section{TABLE 7}

Median Waiting Time: disparity metrics at the TC level, DSA level and region level for different urgency statuses while on the waiting list

\begin{tabular}{lllll|llll}
\hline \multicolumn{7}{c}{ 2006 Policy } & & \multicolumn{3}{l}{ 2016 Policy } \\
\hline & SD & MSBR & Range & IQR & SD & MSBR & Range & IQR \\
\hline Region & & & & & & & & \\
All & 0.05 & 0.10 & 0.16 & 0.12 & 0.07 & 0.13 & 0.21 & 0.13 \\
Status 1A & 0.08 & 0.13 & 0.24 & 0.18 & 0.05 & 0.11 & 0.18 & 0.46 \\
Status 1B & 0.09 & 0.13 & 0.24 & 0.19 & 0.14 & 0.25 & 0.46 & 0.24 \\
Status 2 & 0.03 & 0.03 & 0.10 & 0.04 & 0.08 & 0.20 & 0.25 & 0.14 \\
$D S A$ & & & & & & & & \\
All & 0.08 & 0.24 & 0.34 & 0.17 & 0.10 & 0.21 & 0.36 & 0.23 \\
Status 1A & 0.16 & 0.41 & 0.67 & 0.31 & 0.16 & 0.23 & 1.00 & 0.18 \\
Status 1B & 0.15 & 0.49 & 0.67 & 0.24 & 0.19 & 0.47 & 1.00 & 0.30 \\
Status 2 & 0.04 & 0.23 & 0.25 & 0.04 & 0.13 & 0.52 & 0.60 & 0.15 \\
TC & & & & & & & & \\
All & 0.11 & 0.27 & 0.48 & 0.21 & 0.14 & 0.53 & 0.76 & 0.24 \\
Status 1A & 0.22 & 0.44 & 1.00 & 0.34 & 0.21 & 0.29 & 1.00 & 0.21 \\
Status 1B & 0.19 & 0.81 & 1.00 & 0.29 & 0.25 & 0.53 & 1.00 & 0.43 \\
Status 2 & 0.05 & 0.36 & 0.38 & 0.02 & 0.12 & 0.53 & 0.60 & 0.15 \\
\hline
\end{tabular}

rates are represented by darker shades. Again, the comparison of the two maps reveal that under the 2016 Policy there is an overall improvement of the transplant rate, however, the presence of different shades in both the maps confirms that geographic disparity is present under both the policies and is affecting the same areas in the US. 


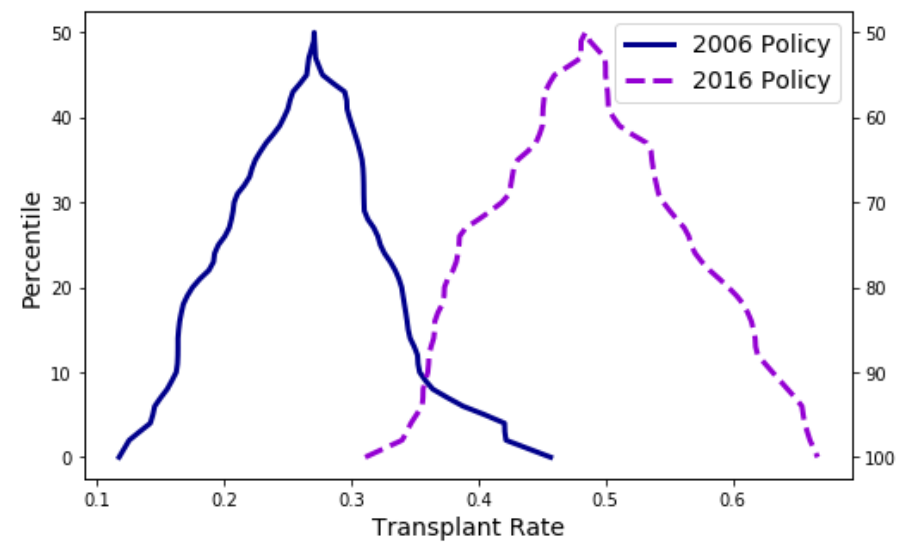

Figure 9. Mountain plot for Transplant Rate
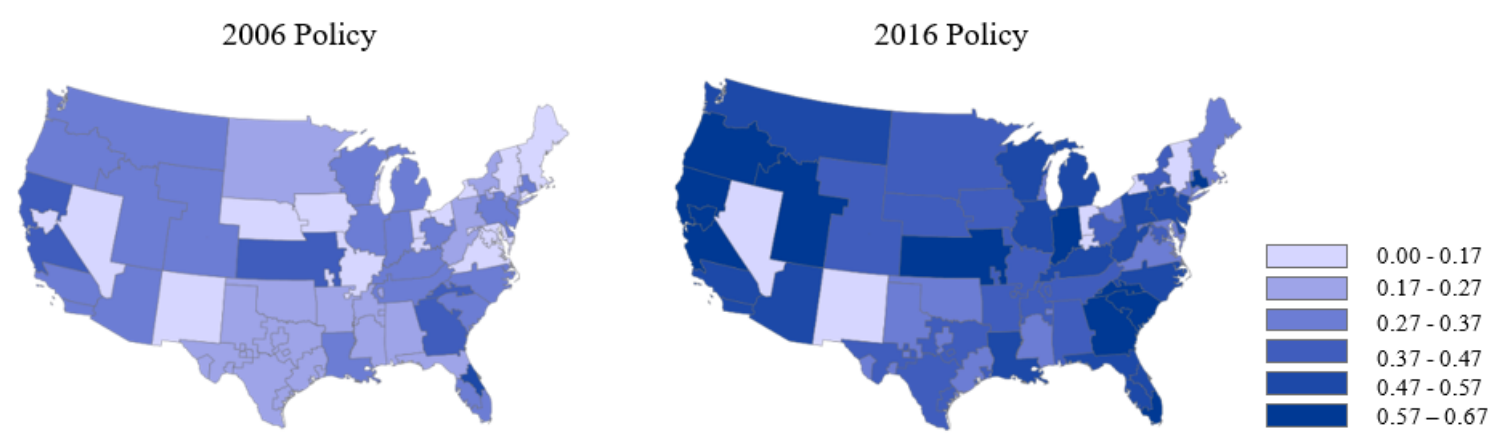

Figure 10. Transplant Rate across DSAs under the 2006 Policy and the 2016 Policy

\subsection{Pre-Transplant Mortality Rate}

Pre-transplant mortality rate is computed as the ratio of the number of patients who died while waiting for a heart to the total number of patients waiting for a transplant. The pre-transplant mortality rate was computed for each geographic level. Figure 11 shows the boxplots of the distribution of the pre-transplant mortality rate computed across each geographic level. At the TC level, the pre-transplant mortality rate ranges between 0.03 and 0.5 under the 2006 Policy, and between 0.02 and 0.25 under the 2016 Policy. The pre-transplant mortality rate, across DSAs, ranges between 0.05 and 0.23 under the 2006 Policy, 
and between 0.03 and 0.21 under the 2016 Policy. Among regions, the pre-transplant mortality rate ranges between 0.08 and 0.14 under the 2006 Policy, and between 0.06 and 0.12 under the 2016 Policy. Table 8 shows the median and average of the pre-transplant mortality rates for different geographic levels, revealing that the pre-transplant mortality rate improves in all geographic levels under the 2016 Policy.

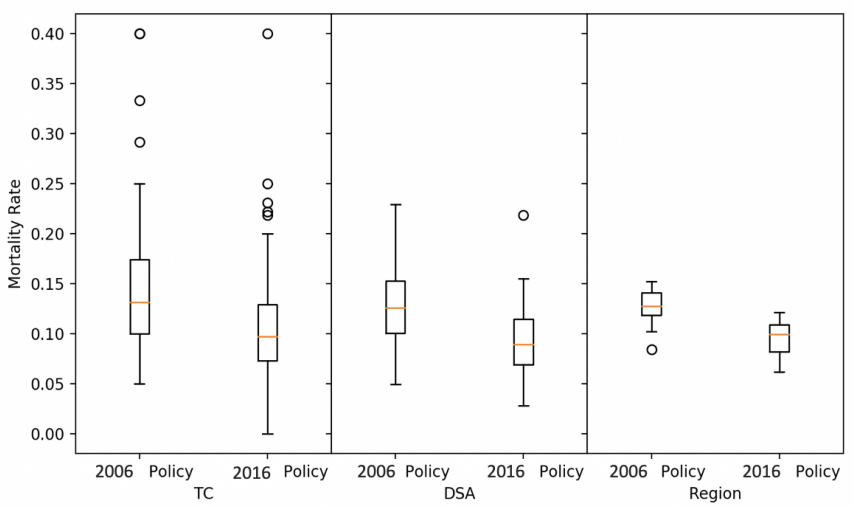

Figure 11. Pre-Transplant Mortality Rate distribution across different geographic levels for the 2006 Policy and the 2016 Policy

Table 9 summarizes the disparity metrics for pre-transplant mortality rate at the TC, DSA, and region levels for different patient urgency statuses. The values of the metrics under the 2006 Policy are very similar to the values computed under the 2016 Policy. Results from the non-parametric Kruskal-Wallis test show that the median values of the pre-transplant mortality rate across DSAs and across regions are not statistically significantly different (at the DSA level: 2006 Policy: $H=52.902, P=0.326,2016$ Policy: $H=53.57, P=0.303$; at the region level: 2006 Policy: $H=15.51, P=0.115,2016$ Policy: $H=11.02, P=0.356)$. These findings are also confirmed by the mountain plots in Figure 12.

Figure 13 shows the choropleth maps of the pre-transplant mortality rate under the 2006 Policy (on the left) and the 2016 Policy (on the right). Higher values are represented by darker shades. Both the maps are in line with the Kruskal-Wallis test: the pre-transplant mortality rate does not show a wide variation across DSAs. 


\section{TABLE 8}

Pre-Transplant Mortality Rate: average and median values at the TC, DSA and region level for different urgency statuses while on the waiting list

\begin{tabular}{|c|c|c|c|c|}
\hline & \multicolumn{2}{|c|}{2006 Policy } & \multicolumn{2}{|c|}{2016 Policy } \\
\hline & Average & Median & Average & Median \\
\hline Region & & & & \\
\hline All & 0.13 & 0.13 & 0.09 & 0.09 \\
\hline Status 1A & 0.05 & 0.04 & 0.04 & 0.03 \\
\hline Status 1B & 0.03 & 0.03 & 0.03 & 0.03 \\
\hline $\begin{array}{l}\text { Status } 2 \\
D S A\end{array}$ & 0.02 & 0.02 & 0.02 & 0.02 \\
\hline All & 0.13 & 0.13 & 0.09 & 0.09 \\
\hline Status 1A & 0.07 & 0.06 & 0.03 & 0.01 \\
\hline Status 1B & 0.05 & 0.03 & 0.03 & 0.02 \\
\hline $\begin{array}{l}\text { Status } 2 \\
T C\end{array}$ & 0.02 & 0.02 & 0.02 & 0.01 \\
\hline All & 0.14 & 0.13 & 0.11 & 0.10 \\
\hline Status 1A & 0.04 & 0.02 & 0.02 & 0.00 \\
\hline Status 1B & 0.05 & 0.02 & 0.04 & 0.00 \\
\hline Status 2 & 0.04 & 0.00 & 0.05 & 0.00 \\
\hline
\end{tabular}

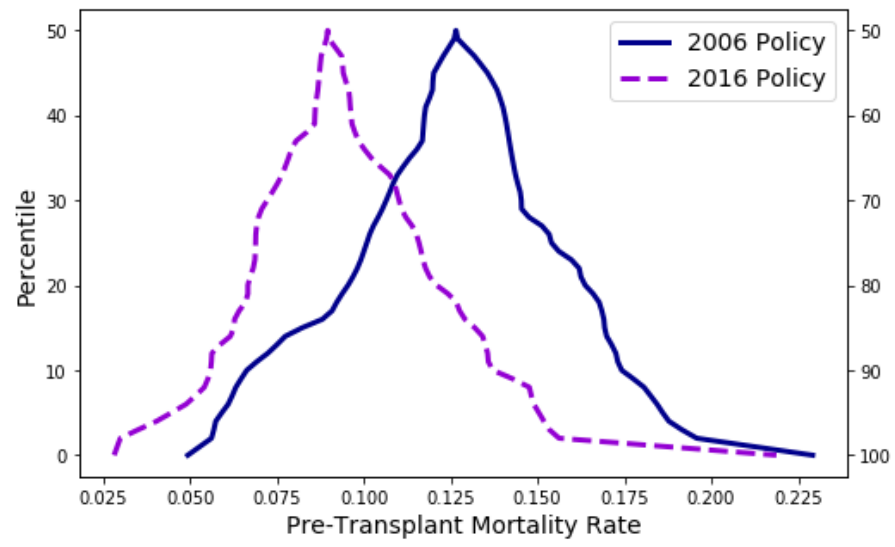

Figure 12. Mountain plot for Pre-Transplant Mortality Rate 


\section{TABLE 9}

Pre-Transplant Mortality Rate: disparity metrics at the TC, DSAs and the region level for different urgency statuses while on the waiting list

\begin{tabular}{lccll|lllll}
\hline \multicolumn{7}{c}{ 2006 Policy } & & \multicolumn{3}{l}{ 2016 Policy } \\
\hline & SD & MSBR & Range & IQR & SD & MSBR & Range & IQR \\
\hline Region & & & & & & & & \\
\hline All & 0.02 & 0.05 & 0.07 & 0.04 & 0.02 & 0.04 & 0.06 & 0.04 \\
Status 1A & 0.02 & 0.03 & 0.05 & 0.04 & 0.02 & 0.04 & 0.06 & 0.04 \\
Status 1B & 0.02 & 0.03 & 0.05 & 0.03 & 0.02 & 0.04 & 0.06 & 0.04 \\
Status 2 & 0.01 & 0.02 & 0.03 & 0.02 & 0.01 & 0.02 & 0.03 & 0.02 \\
DSA & & & & & & & & \\
All & 0.04 & 0.09 & 0.18 & 0.07 & 0.04 & 0.13 & 0.19 & 0.06 \\
Status 1A & 0.07 & 0.10 & 0.29 & 0.12 & 0.05 & 0.22 & 0.25 & 0.07 \\
Status 1B & 0.07 & 0.08 & 0.50 & 0.06 & 0.04 & 0.22 & 0.25 & 0.05 \\
Status 2 & 0.02 & 0.20 & 0.07 & 0.04 & 0.02 & 0.20 & 0.67 & 0.03 \\
TC & & & & & & & & \\
All & 0.07 & 0.14 & 0.48 & 0.09 & 0.07 & 0.11 & 0.48 & 0.07 \\
Status 1A & 0.06 & 0.07 & 0.33 & 0.07 & 0.05 & 0.05 & 0.33 & 0.04 \\
Status 1B & 0.11 & 0.13 & 1.00 & 0.08 & 0.10 & 0.11 & 1.00 & 0.07 \\
Status 2 & 0.12 & 0.13 & 1.00 & 0.06 & 0.11 & 0.17 & 1.00 & 0.04 \\
\hline
\end{tabular}
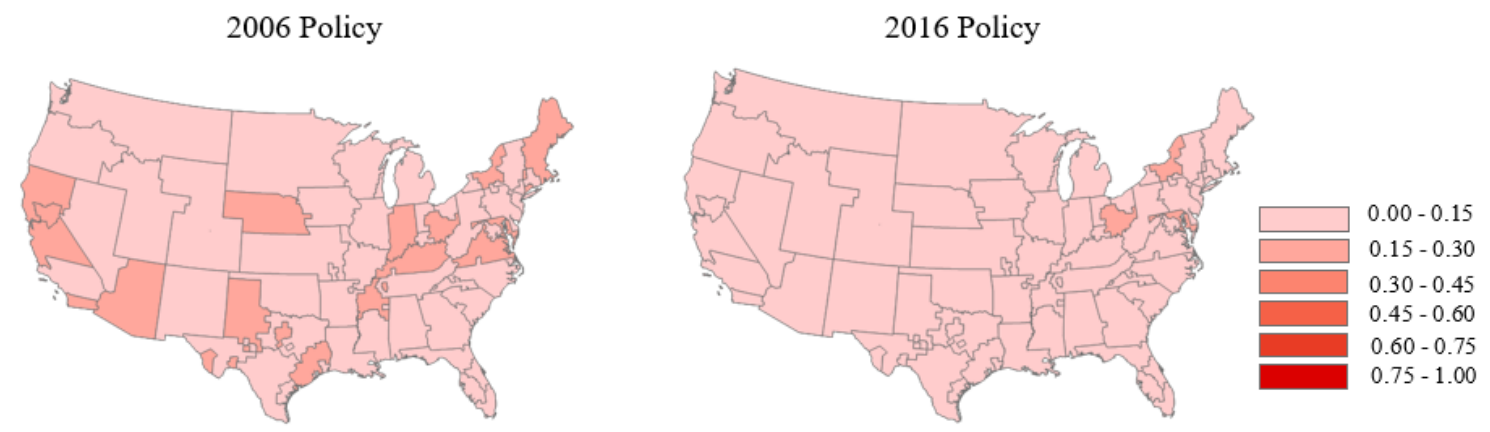

Figure 13. Pre-Transplant Mortality Rate across DSAs under the 2006 Policy and the 2016 Policy 


\subsection{Average Distance Traveled by Donated Hearts}

Average distance traveled by a donated organ was computed at the TC, DSAs and the UNOS region level. Figure 14 shows the boxplots of the distribution of the average distance for each geographic level. At the TC level the average distance ranges between 0 and 412 miles under the 2006 Policy, and between 7 and 691 miles under the 2016 Policy. At the DSA level, the average distance ranges between 7 and 397 miles under the 2006 Policy, and between 8 and 415 miles under the 2016 Policy. At the region level, the average distance ranges between 139 and 346 miles under the 2006 Policy, and between 139 and 333 miles under the 2016 Policy. Table 10 shows the median and average values of the average distance traveled by donated hearts for different geographic levels. This table shows the average distance traveled by donated hearts increases under the 2016 Policy.

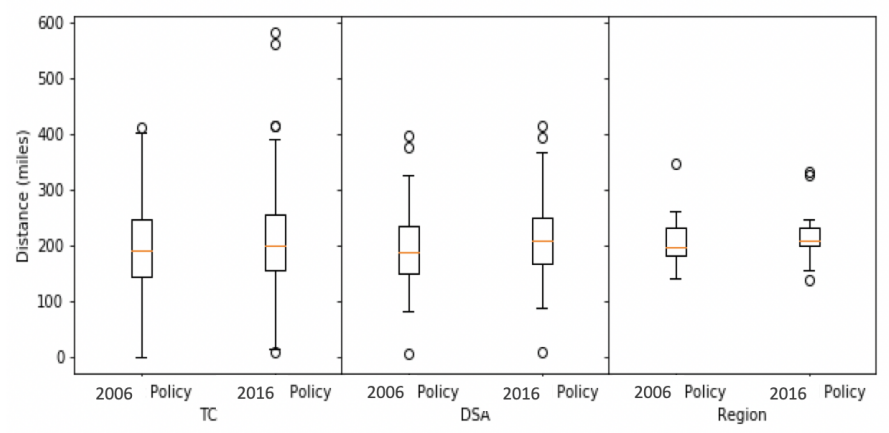

Figure 14. Average Distance distribution across different geographic levels for the 2006 Policy and the 2016 Policy

Table 11 summarizes the disparity metrics for the average distance traveled by donated hearts at the TC, DSA, and region levels for different urgency statuses of the patients while on the waiting list. The disparity metrics reveal that geographic disparity increases with the 2016 Policy for all geographic levels and for all patients statuses (except status 1A patients). Results from the Kruskal-Wallis test show that median values of the average distance traveled by donated hearts 


\section{TABLE 10}

Average Distance Traveled by Donated Hearts: average and median values at the TC, DSAs and the region level for different urgency statuses while on the waiting list

\begin{tabular}{lll|ll}
\hline \multicolumn{3}{c|}{ 2006 Policy } & \multicolumn{2}{l}{ 2016 Policy } \\
\hline Region & Average & Median & Average & Median \\
All & 210 & 197 & 221 & 207 \\
Status 1A & 219 & 211 & 228 & 227 \\
Status 1B & 180 & 163 & 183 & 164 \\
Status 2 & 405 & 387 & 377 & 335 \\
DSA & & & & \\
All & 198 & 189 & 205 & 205 \\
Status 1A & 219 & 204 & 230 & 217 \\
Status 1B & 169 & 156 & 167 & 165 \\
Status 2 & 378 & 343 & 394 & 270 \\
TC & & & & \\
All & 193 & 189 & 219 & 199 \\
Status 1A & 210 & 203 & 226 & 219 \\
Status 1B & 170 & 147 & 178 & 158 \\
Status 2 & 354 & 243 & 350 & 262 \\
\hline
\end{tabular}

across the different geographic levels are statistically significantly different under both the policies (at the TC level: 2006 Policy: $H=355.33, P<0.001,2016$ Policy: $H=348.51, P<0.001$; at the DSA level: 2006 Policy: $H=266.24, P<0.001,2016$ Policy: $H=262.16, P<0.001$; and at the region level: 2006 Policy: $H=86.13$, $P<0.001,2016$ Policy: $H=92.8, P<0.001)$.

These findings are confirmed by the mountain plots in Figure 15. The wide interquartile range in both the mountain plots reveals the presence of geographic disparity under both policies, and since the plot corresponding to the 2016 Policy is shifted to the right, this reveals an increase of the distance under the 2016 Policy.

Figure 16 shows the choropleth maps of the average distance traveled by donated hearts at the DSA level under the 2006 Policy (on the left) and the 2016 Policy (on the right). Longer distances are represented by darker shades. The map 


\section{TABLE 11}

Average Distance Traveled by Donated Hearts: disparity metrics at the TC, DSAs, and region level for different urgency statuses while on the waiting list

\begin{tabular}{lllll|llll}
\hline \multicolumn{5}{c}{ 2006 Policy } & \multicolumn{3}{l|}{ 2016 Policy } \\
\hline Region & SD & MSBR & Range & IQR & SD & MSBR & Range & IQR \\
All & 58 & 89 & 207 & 92 & 60 & 101 & 195 & 122 \\
Status 1A & 59 & 88 & 214 & 89 & 51 & 93 & 177 & 92 \\
Status 1B & 72 & 96 & 252 & 105 & 67 & 124 & 248 & 116 \\
Status 2 & 207 & 345 & 706 & 385 & 209 & 286 & 681 & 361 \\
DSA & & & & & & & & \\
All & 77 & 206 & 391 & 118 & 73 & 211 & 408 & 97 \\
Status 1A & 80 & 222 & 431 & 109 & 70 & 145 & 289 & 126 \\
Status 1B & 85 & 183 & 441 & 97 & 84 & 171 & 381 & 142 \\
Status 2 & 287 & 402 & 1044 & 576 & 356 & 402 & 1727 & 459 \\
TC & & & & & & & & \\
All & 87 & 212 & 412 & 138 & 105 & 236 & 684 & 136 \\
Status 1A & 95 & 226 & 440 & 150 & 113 & 245 & 766 & 174 \\
Status 1B & 122 & 195 & 736 & 176 & 117 & 202 & 561 & 192 \\
Status 2 & 294 & 343 & 1450 & 352 & 295 & 346 & 1730 & 286 \\
\hline
\end{tabular}

on the right is overall darker revealing and increase in the average distance traveled by donated hearts under the 2016 Policy. The variation in color in both maps shows that geographic disparity exists under both policies.

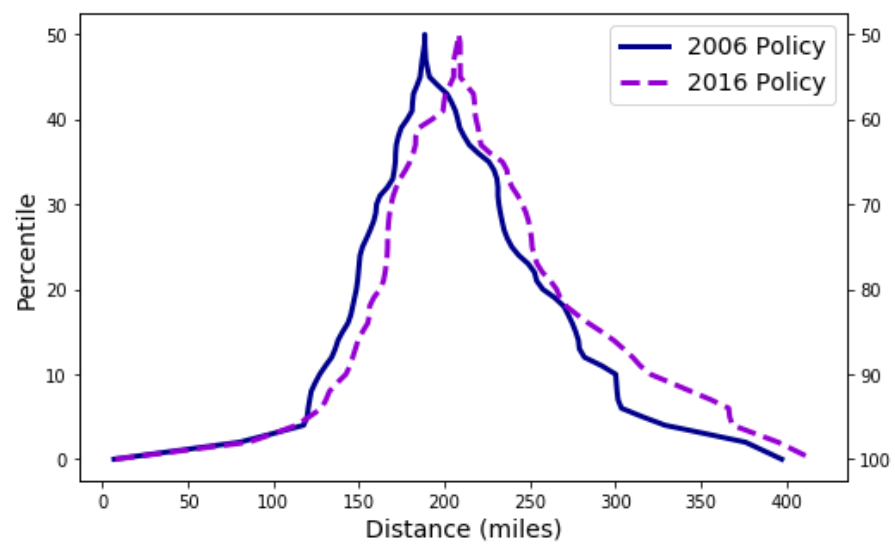

Figure 15. Mountain plot for Average Distance Traveled by Donated Organs 

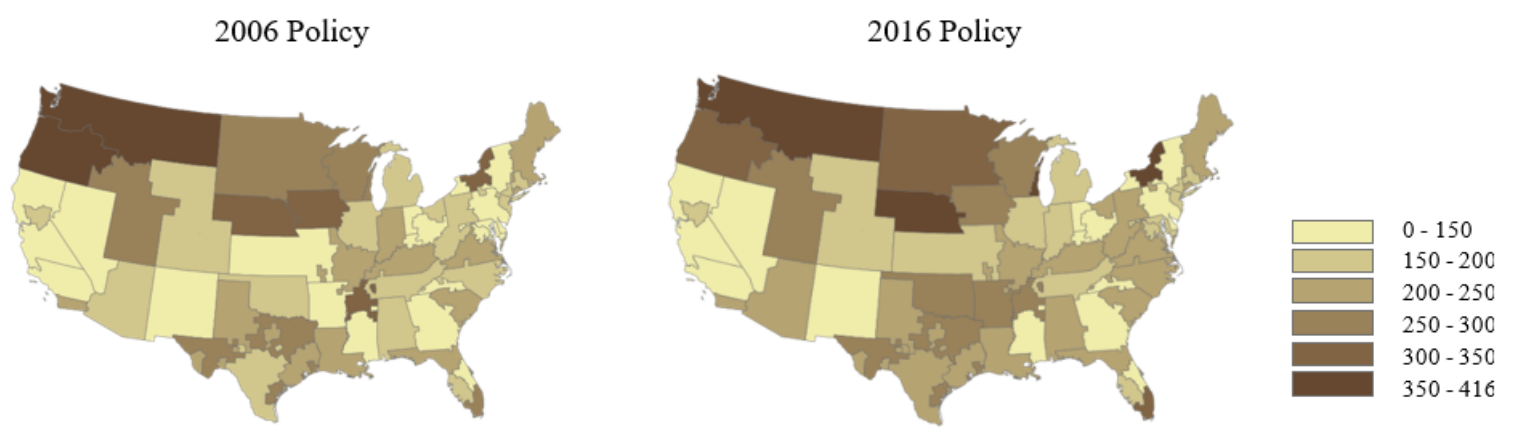

Figure 16. Average Distance (miles) Traveled by Donated Hearts across DSAs under the 2006 Policy and the 2016 Policy

\section{Conclusion}

The heart transplant community has been concerned about high mortality on the transplant wait list. In response to this concern, the Thoracic Organ Transplantation Committee under UNOS approved a different policy for heart allocation in December 2016. While prior literature investigated the overall impact of the 2016 Policy using TSAM (Colvin et al., 2016), implications of the 2016 Policy on geographic disparity have not been evaluated. Such an analysis is warranted since alleviating geographic disparity is one of the stated objectives of the 2016 Policy.

The present study performed a simulation analysis to assess the impacts of the 2016 Policy using an existing simulation software, the Thoracic Simulation Allocation Model (TSAM). The main objective of the analysis was to investigate the impact of the 2016 Policy on geographic disparity measured by four performance indicators: i) access to transplant; ii) waiting time before a transplant; iii) pre-transplant mortality rate; and iv) distance traveled by donated hearts.

The degree of dispersion around the mean performance indicators was large, providing inconclusive results depending on the dispersion measure employed (i.e., SD, MSBR, Range, and IQR). While the predicted size of disparity remains uncertain, our results overall demonstrated that, the geographic disparity will likely 
remain the same with the exception of the waiting time for a transplant. For the waiting time, the results indicated rather consistent reduction in geographic disparity, particularly for the Status 1 patients. The disparity in transplant rate was also improved for the Status 1 patients, although this improvement was achieved by the concomitant reduction in geographic equity among other non-urgent patients. For pre-transplant mortality rate and heart transfer distance, the disparity measures were similar for the two policies.

The 2016 Policy appears to achieve the main goal, i.e., reduced pre-transplant mortality rate. The reduction was seen most significantly among the Status 1 patients (63\%). With broader sharing, average heart transfer distance increased at all levels (increase by 13\%, 4\%, and 5\% at the TC, DSA, and region levels, respectively). The predicted increase was between 7 and 26 miles. Thus, negative impacts of the associated increase in the time between donor-heart retrieval and the transplant (i.e., cold ischemia time) on the likelihood of graft failure or delayed graft function would be negligible. The simulation also showed an expected increase in transplant rate stemming from the increased level of heart utilization. The waiting time was also reduced at all geographic levels $(39 \%, 38 \%$, and $36 \%$ for regions, DSA, and TC levels, respectively). In summary, access to a heart transplant is likely to improve as a result of the 2016 Policy implementation. The 2016 Policy benefits urgent patients in particular, as intended in the 2016 Policy formulation. However, the improvement in geographic equity will remain modest despite it being one of the expected outcomes of the 2016 Policy.

Given the persistent nature of the geographic disparity, a more targeted approach may be necessary to achieve geographic equity in access to transplants. On the open discussion boards regarding the new changes in heart allocation policy, some advocated for varying geographic sharing depending on the population density of DSAs and regions. Designing a broader sharing scheme by taking population density into account with an explicit objective to minimize geographic disparity 
among DSAs or regions could be an effective approach in achieving geographic equity. In Chapter III we will present an optimization model to address geographic disparity in heart allocation system.

Several limitations to our analysis should be noted. First and foremost, there is no defined index in measuring geographic disparity. Our analysis used the metrics that are currently under consideration including the range between the worst and the best location, standard deviation, and the squared average differences from the best location, which gives quantitative results in measuring the disparity. Further exploration and testing to identify adequate metrics and pros and cons of each metric are warranted. The other limitation comes from our use of TSAM in generating simulated data. TSAM uses all patients in the built-in input file in the simulation and does not randomize the patients included in each simulation run. The simulation outputs are deterministic in this sense, limiting the ability to perform further statistical assessment of our simulation outputs using some conventional interval estimates such as confidence intervals (Torlak et al., 2016). The other limitation of TSAM is that it uses an old patient cohort (2009-2011) which might not reflect recent changes in health conditions and demographics. Despite such limitations, the findings of our simulation analysis were fairly consistent in demonstrating the persistent geographic disparity in access to heart transplants, indicating the need for a more direct, targeted effort to reduce disparity.

\section{B Transplant Outcome of Adult Heart Donor with Prior Quality-based Offer Refusal}

There is a donor organ shortage for all transplant organs, including heart, in the United States. Transplantation rates are limited by a shortage of heart donors. Every year nearly 3,000 people per year either die on the transplant waiting list or are removed from the list after waiting so long that they have became too sick to undergo transplant surgery (OPTN, 2018). Despite the increase in the number of 
donated heart organ by 30\% from 2003 to 2007 (Tuttle-Newhall et al., 2009), the number of performed heart transplants over the past two decades has remained steady between 2,000 to 2,500 being performed annually (Kilic et al., 2014). The organ shortage is magnified because many hearts are discarded because of strict selection criteria and concern for regulatory reprimand for less than optimal post-transplant outcomes (Kobashigawa et al., 2017). Only one in three available donor hearts is currently accepted for transplantation, which greatly limits heart transplant rates nationwide (Khush et al., 2015). A study on organ utilization in the U.S. in 2004 reports that $35.8 \%$ of potential diseased heart organs were not recovered because of medical contraindication that was not evident prior to consent or lack of organ viability detected by testing or intraoperatively. Many reasons exist for discarding donor hearts, including demographic and clinical factors (e.g. older age, small size) and co-morbidities (hypertension, diabetes) (Khush et al., 2013).

A study (Khush et al., 2015) closely examined national trends in donor heart acceptance and variation across geographical regions, which shows that organ utilization varies between regions. This variation in organ utilization among regions magnified geographic disparity in access to heart transplants across regions.

In this part of this chapter as a solution to reduce geographic disparity in access to heart transplant, we aim to quantify the geographic disparity in offer heart refusals across OPOs. For improving the organ utilization, we want to answer this question: Are discarded hearts really are not usable (i.e., have worse outcomes)? For this purpose, we closely examined the transplant outcome from transplanted hearts that have been refused one or more times by transplant centers. We compared these results with transplant outcome from transplanted hearts with no prior offer refusals. In the following we discuss the method and the results for our analysis. 


\section{Study Population}

For this study we used UNOS data on hearts organ from 2003 to 2015. During this study period 22,338 heart transplant were performed (excluding candidates with age $<18$, candidates with combined organs need and candidates with re-transplant) and 16,875 hearts have been discarded. The final data set on transplanted hearts after excluding missing data, contains 19,078 transplanted hearts. Among all the transplanted hearts, 13,059 (58\%) were transplanted without any previous quality-based refusal and 9,279 (42\%) were transplanted with at least one quality-based offer refusal. Table 12 shows the number and percentage of hearts on different values of offer refusal rate. For example, 4,796 (52\%) of hearts have been refused between 1 to 5 times by transplant centers before being accepted by a candidate for transplantation.

TABLE 12

Offer refusal rate among transplanted hearts

\begin{tabular}{lccccc}
\hline & $1-5$ & $6-10$ & $11-20$ & $21-30$ & $>30$ \\
\hline Number of Hearts & 4,796 & 1,413 & 1,283 & 599 & 1,185 \\
Percentage of Hearts & $52 \%$ & $15 \%$ & $14 \%$ & $6 \%$ & $13 \%$ \\
\hline
\end{tabular}

\section{Geographic Disparity in Offer Heart Refusal}

In this section we want to examine if the rate of offer heart refusal varies between different OPOs. For each OPO we calculated the total number of hearts that has been offered to a transplant center in that OPO and the number of offers that have been refused by a transplant center in that OPO. The offer refusal rate for each OPO is: the number of offers that has been refused divided by total number of offer. Figure 17 shows the offer refusal rate in each OPO. The offer refusal rate ranges between 0.16 to 0.45 . The variation in color shade in the map 
displays geographic disparity in offer heart refusal among OPOs.



Figure 17. Offer heart refusal rate in each OPO

\section{Discarded Hearts Rate}

Analyzing UNOS data shows that the percentage of discarded hearts increases from 2003 to 2006, and from 2007 this number starts decreasing (Figure 18). This graph shows that over the last 12 years the percentage of discarded hearts have been always greater than 35\%. 37\% of the discarded hearts (6,809 hearts) have been refused less than 10 times by transplant centers before being discarded. Figure 19 shows that, for example, $7 \%$ of the donated hearts (486 hearts) have been discarded without being offer to any patient and $14 \%$ of discarded hearts have been refused by just 1 transplant center before being discarded. 


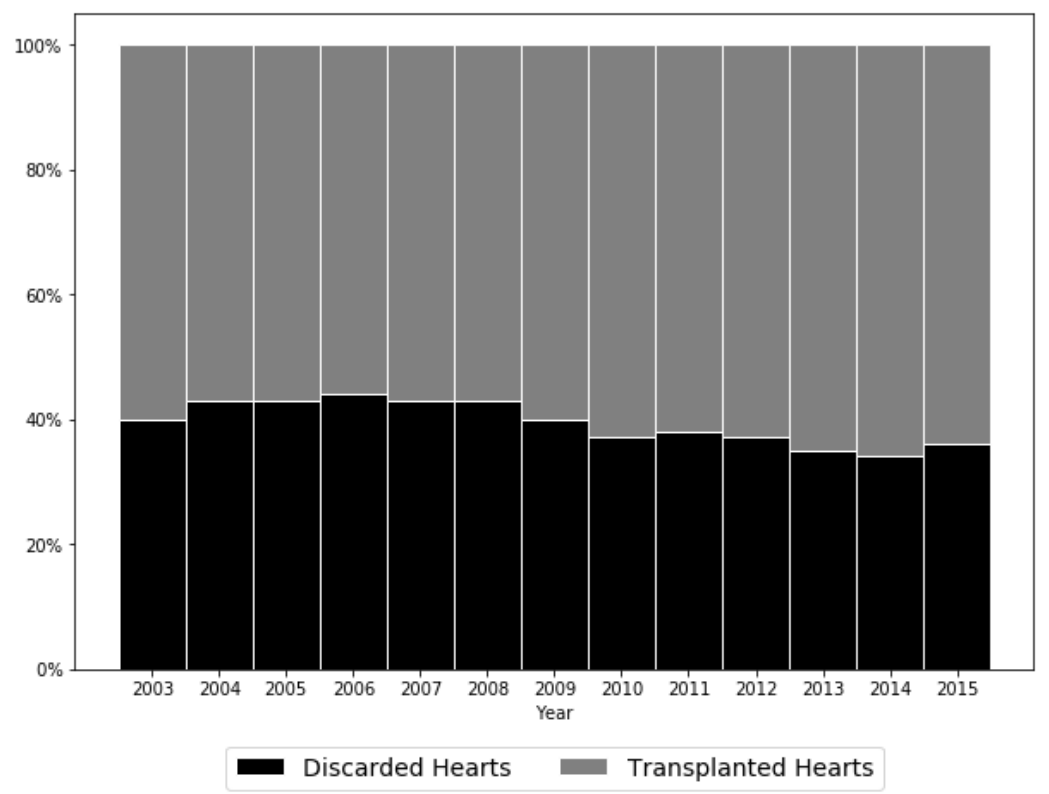

Figure 18. Percentage of transplanted vs. discarded organ hearts by year

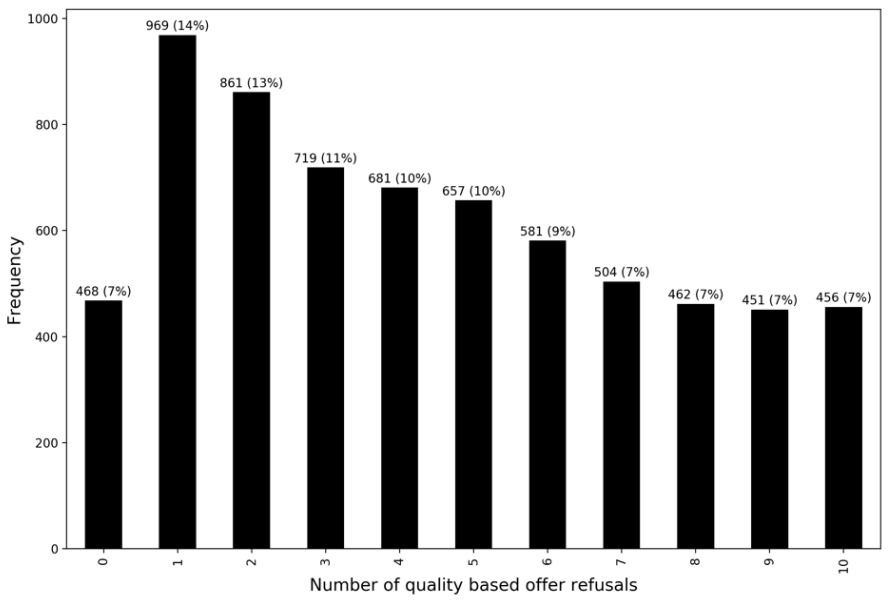

Figure 19. Discarded organ hearts by number of quality-based offer refusals

\section{Transplant Outcome for Hearts with Prior Offer Refusal}

the results from previous sections show that a great percentage of hearts have been discarded in each year, where $37 \%$ of these hearts have been refused for less 
than 10 times before being discarded. The geographic disparity in offer refusal rate across OPOs creates a need to analyze if these discarded hearts have worse outcome. In these section we analyze the transplant outcome from the hearts with previous offer refusal by using the transplanted hearts data from 2003 to 2015 .

\subsection{Statistical Analysis}

We performed a t-test to check if the donors and the transplant centers characteristics are statistically significantly different for transplanted hearts with no prior quality-based refusal and hearts with at least one prior quality-based refusal. Results were considered significant if the p-value was less than 0.05. All reported p-values were two-sided.

We performed a multivariate cox regression analysis to adjust all the covariates listed in Table 13. We used a comprehensive list of risk factors, including cold ischemia time, local donors, number of prior transplants, HLA mismatch level, male recipient, male donor, recipient age $>44$, donor age $>30$, African American donors, and the number of times that a heart has been rejected before transplantation, as the independent variables. Patient death was the dependent variable. The percentage of missing value for HLA mismatch level was $13 \%$ and for all other variables was below 3\%. For the purpose of analysis, we deleted all the instances that contains missing values. 90-days, 1-year, and 3-years post-transplant survival were calculated using Kaplan-Meier analysis. The primary outcome measure was patient death. For this analysis, time to death was assessed as time from the time of transplant to the time of death. Patients lost to follow-up or alive on December 31, 2015, were censored at the date of last know follow-up. 
TABLE 13

Transplant center and donor demographic for transplanted hearts with offer refusal and without any offer refusal

\begin{tabular}{|c|c|c|c|}
\hline & $\begin{array}{c}\text { No Refusal } \\
(\mathrm{n}=13,059,58 \%)\end{array}$ & $\begin{array}{c}\text { Offer Refusal } \\
(\mathrm{n}=9,276,42 \%)\end{array}$ & P-value \\
\hline Age $>50, \mathrm{n}(\%)$ & $640(5 \%)$ & $1,364(14 \%)$ & $<0.001$ \\
\hline Male, n(\%) & $9,951(76 \%)$ & $6,009(64 \%)$ & $<0.001$ \\
\hline \multicolumn{4}{|l|}{ Race, $\mathrm{n}(\%)$} \\
\hline White & $8,428(64 \%)$ & $6,199(66 \%)$ & $<0.001$ \\
\hline African American & $1,926(14 \%)$ & $1,449(15 \%)$ & 0.074 \\
\hline Hispanic & $2,313(17 \%)$ & $1,375(14 \%)$ & $<0.001$ \\
\hline Asian & $215(1.6 \%)$ & $154(1.7 \%)$ & 0.939 \\
\hline History of hypertension, $\mathrm{n}(\%)$ & $1,246(9 \%)$ & $1,836(19 \%)$ & $<0.001$ \\
\hline ECD donor, n(\%) & $216(1.6 \%)$ & $510(5.5 \%)$ & $<0.001$ \\
\hline \multicolumn{4}{|l|}{ Cause of Death, $n(\%)$} \\
\hline Anoxia & $1,480(11 \%)$ & $2,304(24 \%)$ & $<0.001$ \\
\hline Cerebrovascular/Stroke & $2,485(19 \%)$ & $2,550(27 \%)$ & $<0.001$ \\
\hline Head trauma & $8,805(67 \%)$ & $4,083(44 \%)$ & $<0.001$ \\
\hline CNS tumor & $71(0.54 \%)$ & $105(1.1 \%)$ & $<0.001$ \\
\hline Unknown & $216(1.7 \%)$ & $237(2.6 \%)$ & $<0.001$ \\
\hline \multicolumn{4}{|l|}{ Share type, n (\%) } \\
\hline Local & $9,900(75 \%)$ & $3,730(40 \%)$ & $<0.001$ \\
\hline Regional & $1,609(12 \%)$ & $1,925(20 \%)$ & $<0.001$ \\
\hline National & $1,540(11 \%)$ & $3,621(39 \%)$ & $<0.001$ \\
\hline Cold ischemia time (hrs), mean & 3.07 & 3.42 & $<0.001$ \\
\hline Transfer distance (mile), mean & 124.8 & 243.6 & $<0.001$ \\
\hline HLA mismatch level, mean & 4.62 & 4.62 & 0.933 \\
\hline
\end{tabular}




\subsection{Results}

Multivariate Cox Regression Analysis of Graft Failure Table 14 shows the multivariate Cox regression analysis of graft failure. The results from cox regression help in recognizing the factors that effect graft failure. The most important point taken from the Table 14 is that hearts that previously have been refused between 1-5 times $(\mathrm{HR}=1.041, \mathrm{p}=0.119)$ and between 6 -10 times $(1.088, \mathrm{p}=0.167)$ did not have a higher risk of graft failure.

Regarding the factors that impact graft survival; in terms of year of the transplant, the transplant recipients who received a transplant in years 2003 $(\mathrm{HR}=1.095, \mathrm{p}=0.048), 2006(\mathrm{HR}=1.107, \mathrm{p}=0.018), 2010(\mathrm{HR}=0.870, \mathrm{p}=0.009)$, $2011(\mathrm{HR}=0.854, \mathrm{p}=0.007)$ and $2013(\mathrm{HR}=0.859, \mathrm{p}=0.024)$ had a higher risk of graft failure. The recipients with a prior history of transplant had a decreased long-term transplant survival $(\mathrm{HR}=1.291, \mathrm{p}<0.001)$. HLA mismatch level has significant effect on graft failure $(\mathrm{HR}=1.032, \mathrm{p}=0.011)$. Donor heart cold ischemia time and local donor were the major factors $(\mathrm{HR}=1.094, \mathrm{p}<0.001$ and $\mathrm{HR}=1.114$, $\mathrm{p}=0.001)$. Older recipients $(>44)$ were associated with a higher risk of graft failure $(\mathrm{HR}=0.927, \mathrm{p}<0.001)$. Donor age $>30$ years also was a primary significant factor affecting long term survival $(\mathrm{HR}=1.207, \mathrm{p}<0.001)$. Finally, for number of quality based refusals, grafts with 11 to 20 previous offer refusals $(H R=1.205, p=0.004), 21$ to 30 previous offer refusals $(\mathrm{HR}=1.242, \mathrm{p}=0.018)$ and more than 30 previous offer refusal $(\mathrm{HR}=1.207, \mathrm{p}=0.011)$ had higher risk of graft failure. 
TABLE 14

Multivariate Cox regression analysis of graft failure

\begin{tabular}{|c|c|c|}
\hline & $\begin{array}{c}\text { Multivariate Hazard } \\
\text { Ratio }\end{array}$ & P-value \\
\hline Transplant year: 2003 & $1.095(1.000-1.199)$ & 0.048 \\
\hline Transplant year: 2006 & $1.107(1.017-1.205)$ & 0.018 \\
\hline Transplant year: 2010 & $0.870(0.784-0.967)$ & 0.009 \\
\hline Transplant year: 2011 & $0.854(0.762-0.957)$ & 0.00 \\
\hline Transplant year: 2013 & $0.859(0.753-0.980)$ & 0.024 \\
\hline Cold ischemic time & $1.094(1.065-1.124)$ & $<0.001$ \\
\hline Local donor & $1.114(1.045-1.186)$ & 0.001 \\
\hline Number of previous transplants & $1.291(1.177-1.416)$ & $<0.001$ \\
\hline HLA mismatch level & $1.032(1.007-1.057)$ & 0.011 \\
\hline Male recipient & $0.941(0.884-1.001)$ & 0.054 \\
\hline Male donor & $0.992(0.934-1.053)$ & 0.795 \\
\hline Recipient age $>44$ & $0.923(0.871-0.979)$ & 0.008 \\
\hline Donor age $>30$ & $1.250(1.182-1.323)$ & $<0.001$ \\
\hline African American donor & $1.059(0.985-1.138)$ & 0.119 \\
\hline Number of refusals: $1-5$ & $1.042(0.971-1.118)$ & 0.248 \\
\hline Number of refusals: $6-10$ & $1.088(0.965-1.226)$ & 0.167 \\
\hline Number of refusals: $11-20$ & $1.205(1.063-1.366)$ & 0.004 \\
\hline Number of refusals: $21-30$ & $1.242(1.038-1.486)$ & 0.018 \\
\hline Number of refusals: above 30 & $1.207(1.043-1.384)$ & 0.011 \\
\hline
\end{tabular}

Multivariate Cox Regression Analysis of Patient Failure In this section by using multivariate cox regression we want to recognize the factors that effect patient failure. Table 15 shows the multivariate Cox regression analysis of post-transplant 
mortality risk. Important point taken from Table 15 is that grafts with 1-5 prior offer refusals does not have higher risk of patient failure $(\mathrm{HR}=1.075, \mathrm{p}=0.064)$.

Regarding the factors that impact patient survival; in terms of year of transplant, the transplant recipients who received a transplant in 2011 (HR=0.847, $\mathrm{p}=0.009$ ) had a higher risk of failure. Donor heart cold ischemia time and local donor were the major factors ( $\mathrm{HR}=1.091, \mathrm{p}<0.001$ and $\mathrm{HR}=1.092, \mathrm{p}=0.011)$. The recipients with a previous transplant had a decreased transplant survival $(\mathrm{HR}=1.272, \mathrm{p}<0.001)$. HLA mismatch level has significant effect on patient survival $(\mathrm{HR}=1.026, \mathrm{p}=0.050)$. Older recipients $(>44)$ were associated with a higher risk of failure $(\mathrm{HR}=0.927, \mathrm{p}=0.025)$. Donor age $>30$ years also was a primary significant factor affecting long term survival $(\mathrm{HR}=1.207, \mathrm{p}<0.001)$. Finally, for number of quality based refusals, recipients of grafts with 6 to 10 previous offer refusals $(\mathrm{HR}=1.141, \mathrm{p}=0.036), 11$ to 20 previous offer refusals $(\mathrm{HR}=1.256, \mathrm{p}=0.001), 21$ to 30 previous offer refusal $(\mathrm{HR}=1.321, \mathrm{p}=0.002)$ and more than 30 previous offer refusal $(\mathrm{HR}=1.231, \mathrm{p}=0.004)$ had higher risk of mortality. 


\section{TABLE 15}

Multivariate Cox regression analysis of post-transplant mortality risk

\begin{tabular}{|c|c|c|}
\hline & $\begin{array}{c}\text { Multivariate Hazard } \\
\text { Ratio }\end{array}$ & P-value \\
\hline Transplant year: 2003 & $1.086(0.986-1.197)$ & 0.092 \\
\hline Transplant year: 2006 & $1.093(0.998-1.198)$ & 0.055 \\
\hline Transplant year: 2010 & $0.906(0.811-1.014)$ & 0.087 \\
\hline Transplant year: 2011 & $0.847(0.748-0.958)$ & 0.009 \\
\hline Transplant year: 2013 & $0.915(0.796-1.053)$ & 0.217 \\
\hline Cold ischemic time & $1.091(1.059-1.124)$ & $<0.001$ \\
\hline Local donor & $1.092(1.020-1.170)$ & 0.011 \\
\hline Number of previous transplant & $1.272(1.142-1.414)$ & $<0.001$ \\
\hline HLA mismatch level & $1.026(1.000-1.053)$ & 0.050 \\
\hline Male recipient & $0.966(0.902-1.035)$ & 0.336 \\
\hline Male donor & $0.965(0.903-1.031)$ & 0.299 \\
\hline Recipient age $>44$ & $0.927(0.867-0.990)$ & 0.025 \\
\hline Donor age $>30$ & $1.207(1.139-1.280)$ & $<0.001$ \\
\hline African American donor & $1.069(0.988-1.156)$ & 0.097 \\
\hline Number of refusal: $1-5$ & $1.075(0.995-1.161)$ & 0.064 \\
\hline Number of refusal: $6-10$ & $1.141(1.008-1.294)$ & 0.036 \\
\hline Number of refusal: $11-20$ & $1.256(1.104-1.428)$ & 0.001 \\
\hline Number of refusal: $21-30$ & $1.321(1.104-1.581)$ & 0.002 \\
\hline Number of refusal: above 30 & $1.231(1.066-1.421)$ & 0.004 \\
\hline
\end{tabular}

Survival Analysis to Graft Failure Using Kaplan-Meier analysis we compared the transplant outcome for 90-day 1- and 3-year for the transplanted hearts with prior offer refusal and hearts with no prior offer refusal. In general, among the 
26,563 transplanted grafts $40 \%$ of them (10,681 grafts) had at least one previous quality-based offer refusal. The 90-days survival was similar among cohorts independent of grafts, at $94 \%$ for the grafts with no previous offer refusal compared to $93 \%$ with at least one quality based offer refusal. The 1-year survival was also similar at $89 \%$ with no quality based offer refusal and $88 \%$ with at least one quality based offer refusal. 3-year survival for transplanted grafts with no quality based offer refusal is $82 \%$ and for grafts with at least one quality based offer refusal is $80 \%$ (Figure 20). The 90-day, 1- and 3-year survival are displayed in Table 16. Figure 21 shows the 3-year survival curve for transplanted hearts with different number of prior offer refusal. This figure shows that the survival curve for the hearts with 1-5 and 6-10 prior offer refusals is very close to the survival curve for the hearts with no prior offer refusal.

\section{TABLE 16}

90-days 1- and 3-Year survival as stratified by number of previous quality-based offer refusals

\begin{tabular}{lccc}
\hline & $\begin{array}{c}\text { 90-day } \\
\text { survival }(\%)\end{array}$ & $\begin{array}{c}\text { 1-year survival } \\
(\%)\end{array}$ & $\begin{array}{c}\text { 3-year survival } \\
\text { Number of refusals: } 0\end{array}$ \\
\hline Number of refusals: 1-5 & 94 & 89 & 82 \\
Number of refusals: 6-10 & 92 & 88 & 81 \\
Number of refusals: 11-20 & 91 & 89 & 82 \\
Number of refusals: 21-30 & 92 & 87 & 79 \\
Number of refusals: above 30 & 93 & 87 & 78 \\
\hline
\end{tabular}




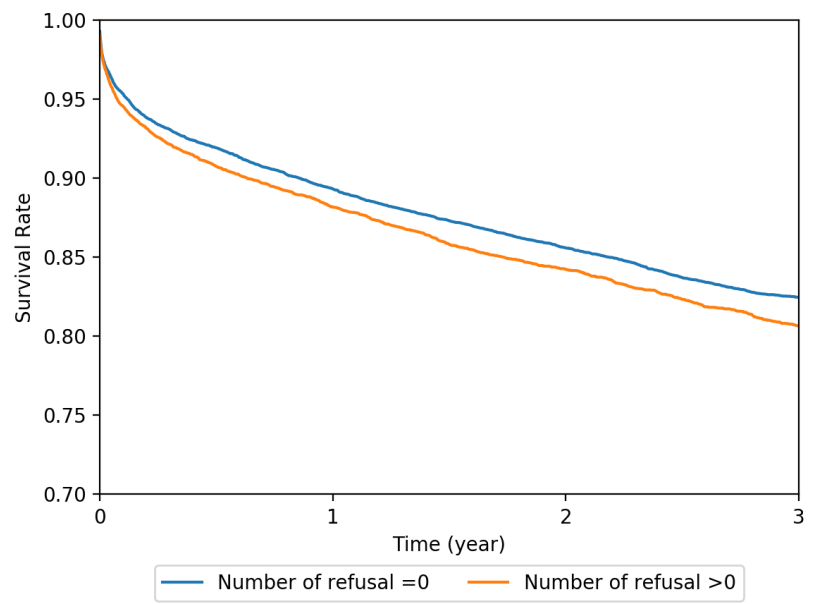

Figure 20. Kaplan-Meier analysis demonstrating cumulative survival rate for grafts with no previous quality based offer refusal and grafts with at least one previous quality based offer refusal and (log rank, $\mathrm{p}<0.001$ )

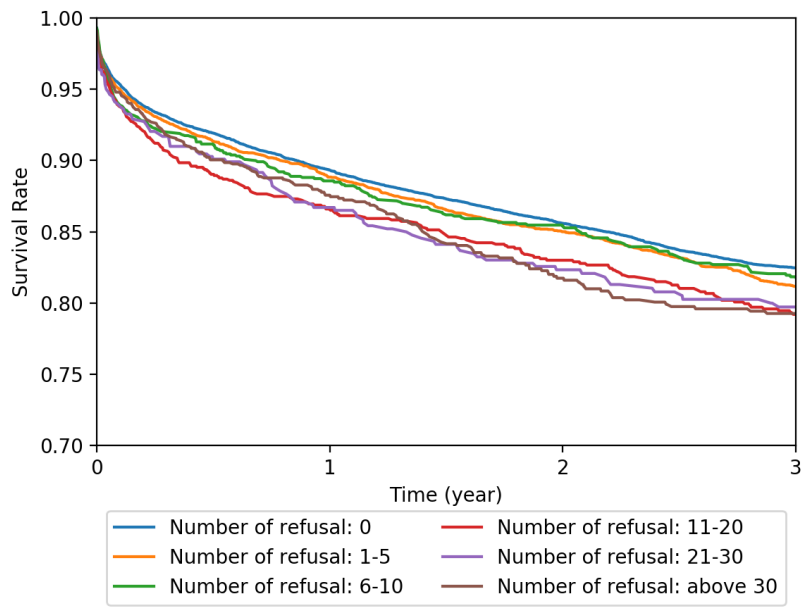

Figure 21. Kaplan-Meier analysis demonstrating cumulative survival rate for grafts stratified by the number of previous quality-based offer refusals (log rank, $\mathrm{p}<0.001$ ) 


\section{Conclusion}

One of the main causes of the geographic disparity is the uneven distribution of supply and demand across the country. The gap between supply and demand become even larger because of the variability in organ usage rate, organ waste and other factors which influence the effective use of donated organs (Mathur et al., 2010). Our analysis showed that disparity in offer refusal rate exists among OPOs and it ranged from 0.16 to 0.45 . To deal with the widening gap between supply and demand for heart transplants, researchers have suggested to increase the donor pool by using sub-optimal heart organs.

The main focus of our study was to analyze weather the discarded organs have worse outcome and weather we can use a percentage of these organs to increase access to heart transplants. To do so, we analyzed the transplant outcome from grafts with prior offer refusal and compared their outcome to grafts with no previous offer refusals. We used cox regression to analyze if these two groups of grafts, statistically, have different outcome in terms of graft and patient failure. The results from our cox regression indicated that grafts with 1-10 times prior offer refusals did not have different outcomes from the grafts with no prior offer refusal (p-value $>0.036)$. Additionally, the results from Kaplan-Meier analysis indicated that organs with 1-10 times prior offer refusal have the same 3-year transplant outcome (81\%). It should be noted that from 16,875 discarded hearts, 6,809 (40\%) of them have been refused less than 10 times by transplant centers.

The variation in organ utilization across the country causes an unbalanced distribution of supply and demand, which ultimately can cause geographic disparity in access to heart transplants. Regions with higher offer refusals reject most offers and wasting a great percentage of their supply for organs. Our analyze indicated that a great percentage (potentially 40\%) of hearts that have been discarded under current practice could have been utilized without compromising transplant outcome. 


\section{CHAPTER III}

\section{OPTIMIZATION MODEL TO ADDRESS GEOGRAPHIC DISPARITY IN ACCESS TO HEART TRANSPLANTS}

\section{A Introduction}

Heart failure is a growing health problem affecting nearly 6 million people in the United States (Go et al., 2013a), with approximately 200,000 patients suffering from a progressed to end-stage or Stage D heart disease (AFZAL AMMAR et al., 2007). Heart transplantation is the definitive therapy for end-stage heart disease patients. For the patients who underwent a heart transplant from 2007 through 2009, the 1-year survival rate was 88.8\%, while the 3-year and 5-year survival rates were $82 \%$ and $76 \%$, respectively (Colvin-Adams et al., 2014). In the United States,

organ allocations for transplantation have been administered by the United Network for Organ Sharing (UNOS). UNOS established the Organ Procurement and Transplantation Network (OPTN) in 1984 to coordinate across 58 Organ Procurement Organizations (OPOs) which are grouped into 11 regions. Each of these OPOs is responsible for acquiring and distributing organs procured within their service area which is known as the Donor Service Area (DSA). OPTN's mission is to ensure a fair and equitable allocation of organs.

Organ allocation protocols vary depending on the organ type. A guiding principle for heart allocation is to prioritize those in greatest need within an acceptable geographic distance. The typical set of zones is defined through increasing concentric circles (see Figure 22) with radii of 250 miles (Local), 500 miles (Zone A), 1,000 miles (Zone B), 1,500 miles (Zone C), 2,500 miles (Zone D), 
and outside 2,500 miles (Zone E). Thus, transplant candidates registered at the transplant centers located within the donor hospital's local area receive offers first, followed by the candidates at the transplant centers in the successive zones.

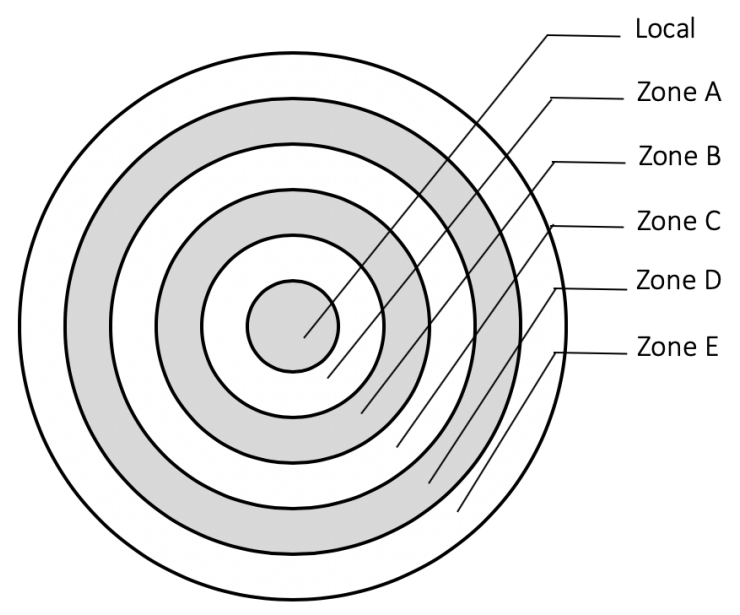

Figure 22. Current homogeneous zonal structure composed of six zones around donor hospitals in the heart allocation system.

Prior studies have reported various disparities in access to heart transplants. In particular, disparity associated with patient locations (geographic disparity) has been identified as an issue in the current allocation system (Kobashigawa, 2014; Singh et al., 2014). Another study showed that the number of transplants performed on higher urgency candidates varies significantly from $37 \%$ to $86 \%$ across geographic regions (Meyer et al., 2015). This is contrary to the founding principles of OPTN, which focus on recipient chance equality.

In 2016 the OPTN charged its Thoracic Organ Transplantation Committee with proposing changes to the 2006 allocation system. The new policy took effect in October 2018. Despite the known geographic disparity in the system, the majority of the proposed changes in the heart allocation policy focused on the prioritization of transplant candidates according to their severity status, rather than geographic factors (Kobashigawa, 2014; Singh et al., 2014; OPTN, 2016; Fudim, 2019). The new policy groups adult recipients (age $\geq 18$ ) into six severity statuses (status 1 to 
6, with status 1 corresponding to the most severe patients) in contrast with the three severity levels of the previous policy (status 1A, status 1B, and status 2). Additionally, the newly approved heart allocation policy altered the sequence of allocation for the most urgent adult candidates by so-called broader sharing: hearts from deceased donors age 18 years or older will be offered first to compatible adult status 1 and pediatric (age $<18)$ status $1 \mathrm{~A}$ candidates within a 500 mile radius from the donor hospital, then to compatible adult status 2 candidates within a 500 mile radius. If no matches are made for these candidates, hearts will be offered to the candidates in lower urgency statuses within a 250 mile radius (OPTN, 2016; Fudim, 2019).

In a previous study (Karami et al., 2019), which is presented in ChapterII, we showed that geographic disparity in access to heart transplants is likely to persist with the new policy. Additionally, the transplant community has voiced a number of concerns regarding the new heart allocation proposal (OPTN, 2016). The main concern is that the new allocation policy might not be sufficient to reduce geographic disparity in access to heart transplants, because the current zonal structure is homogeneous across donor hospitals and does not take into account the regional heterogeneity of transplant centers and of population density. In this context, a heterogeneous zonal structure has been suggested as a possible solution to overcome these limits (OPTN, 2016). A heterogeneous zonal structure would allow each donor hospital to have a different number of zones with different radii.

In light of this, we proposed a novel mathematical optimization model to formulate the Boundary Allocation Problem (BAP) to assign different zonal structures to donor hospitals and minimize geographic disparity in access to heart transplants across transplant centers. In our analysis we aimed to answer the following questions:

1. How much does a heterogeneous zonal structure reduce geographic disparity in access to heart transplants? 
2. How much does a heterogeneous zonal structure change the average distance traveled by organs?

3. How heterogeneous should the zonal structure around donor hospitals be to minimize geographic disparity?

4. How robust is a heterogeneous zonal structure with respect to changes in supply and demand over time?

Although mathematical optimization is recognized as an important tool in decision making to address geographic disparity in the allocation system of other organs (Zenios et al., 2000; Stahl et al., 2005; Kong et al., 2010; Demirci et al., 2012; Gentry et al., 2013; Davis et al., 2015; Ata et al., 2016), a mathematical optimization approach to address geographic disparity in the heart allocation system has not been explored yet, and to the best of our knowledge this is the first attempt in this direction. The only existing study is by Hasankhani and Khademi (2017), who used a simulation model to evaluate alternative allocation systems to reduce geographic disparity in access to heart transplants. They evaluated three different strategies; (i) combining the three immediate zones in the current homogeneous zonal structure into one zone, (ii) altering the prioritization across patients' medical status, and (iii) giving higher priority to patients with longer waiting times. What makes our study different is that we use an optimization model to design the heterogeneous zonal structure as opposed to the aforementioned study, which arbitrary combined the zones in the current homogeneous zonal structure.

The remainder of the paper is structured as follows. In the next section, we formally define the Boundary Allocation Problem. The mathematical formulation is provided in Section C. Our experimental design is described in Section E, and results are presented in Section F. Finally, in Section H and I we further discuss the results and limitations of our study, as well as possible next steps. 


\section{B Problem Definition}

In this section, we formally define the Boundary Allocation Problem. The definition of zones, zonal structure, access level, and access level disparity is provided first.

\section{Zonal Structure Definition}

Let $H=\{1,2, \ldots, m\}$ denote the set of $m$ donor hospitals, and

$T=\{1,2, \ldots, n\}$ the set of $n$ transplant centers. Let $\mathcal{C}=\left\{c_{0}, c_{1}, \ldots, c_{q}\right\}$ be a set of $q$ predefined concentric circles around a donor hospital, such that circle $c_{s}$ is included in circle $c_{k}$ for each $s<k$, and $c_{0}$ is corresponding to the point in the center of the innermost circle. We denote the corresponding index set as $C=\{0,1,2, \ldots, q\}$. As an example consider Figure 23 which shows donor hospital $j$, with four predefined concentric circles $\mathcal{C}=\left\{c_{0}, c_{1}, c_{2}, c_{3}\right\}$.

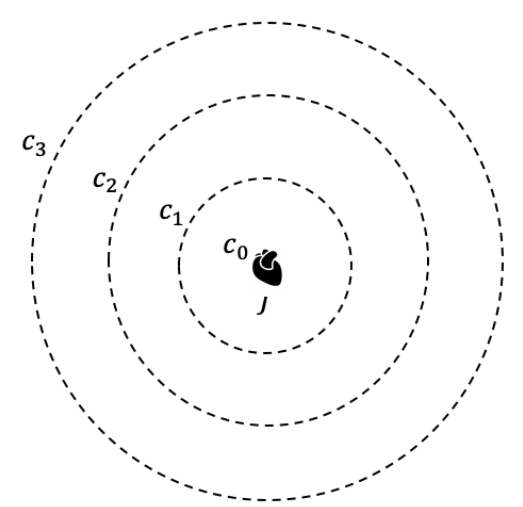

Figure 23. Set of four predefined circles around a donor hospital.

We define a zone as the area between an ordered pair of circles, and a zonal structure as a collection of contiguous zones associated with a given donor hospital. Formally:

DEFINITION 1 A zone $z$ around a donor hospital is an ordered pair of circles, i.e., $z=\left(c_{s}, c_{k}\right), s<k$, where $c_{s}$ is the inner circle of the zone, and $c_{k}$ is the outer 
circle of the zone.

DEFINITION 2 A zonal structure of a donor hospital $j \in H$ is a collection $\left\{z_{1 j}, z_{2 j}, \ldots, z_{\ell j}\right\}$ of $\ell$ zones associated with the donor hospital.

DEFINITION 3 The innermost zone $z_{1}$ is defined by pairing the fictitious circle $c_{0}$ with another predefined circle $c_{s}$, i.e., $z_{1}=\left(c_{0}, c_{s}\right), s \neq 0$ and $s \leq q-\ell+1$. A proper zonal structure around each donor hospital must be composed of contiguous zones:

DEFINITION 4 A zonal structure $\left\{z_{1 j}, z_{2 j}, \ldots, z_{\ell j}\right\}$ around donor hospital $j \in H$ is contiguous if the outer circle of $z_{i j}$ coincides with the inner circle of $z_{i+1 j}$, $\forall i=1, \ldots, \ell-1$. Going back to our example, assume that by selecting from a predefined set of four circles (Figure 23) we want to design a zonal structure around donor hospital $j$ which is composed of two zones, i.e., $\left\{z_{1 j}, z_{2 j}\right\}$. Figure 24 shows the possible zonal structures. For example, if $z_{1 j}$ is defined as the area between $c_{0}$ and $c_{1}$, then the outer circle of $z_{1 j}$ is $c_{1}$. In this case, $z_{2 j}$, can be defined as either the area between $c_{1}$ and $c_{2}$ (dark grey area in Figure $\left.24(\mathrm{a})\right)$, or the area between $c_{1}$ and $c_{3}$ (dark grey area in Figure $24(\mathrm{~b})$ ). Note that $z_{2 j}$ cannot be defined as the area between $c_{2}$ and $c_{3}$, since $z_{1 j}$ and $z_{2 j}$ would not be contiguous. On the other hand, if $z_{1 j}$ is defined as the area between circle $c_{0}$ and $c_{2}$ (light grey area in Figure 24(c)), then the only possible configuration for $z_{2 j}$ would be the area between $c_{2}$ and $c_{3}$ (dark grey area in Figure 24(c)).

\section{Access Level}

Transplant rate (Stewart et al., 2018), waiting time (Davis et al., 2015), and supply to demand ratio (Reese et al., 2014; Lewis et al., 2014; Haugen et al., 2019) were commonly used by other researchers for measuring geographic disparity in access to organ transplants. In particular, geographic disparity in access to 


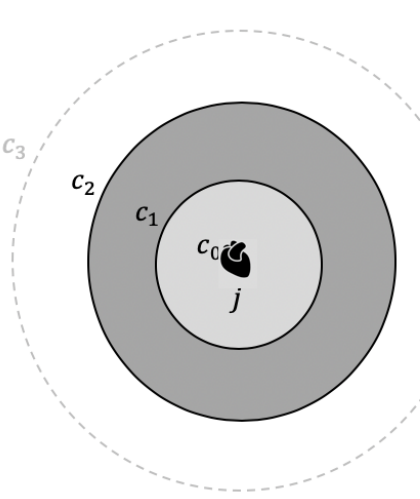

(a)

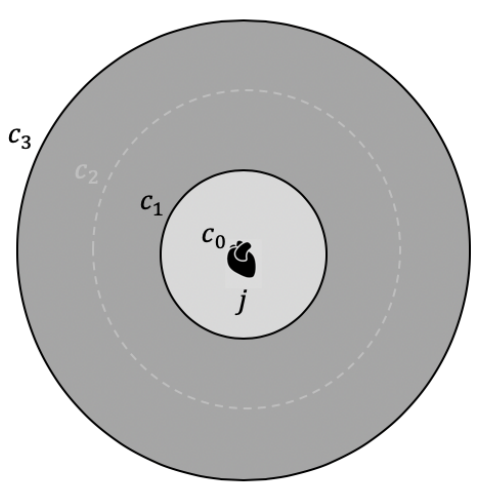

(b)

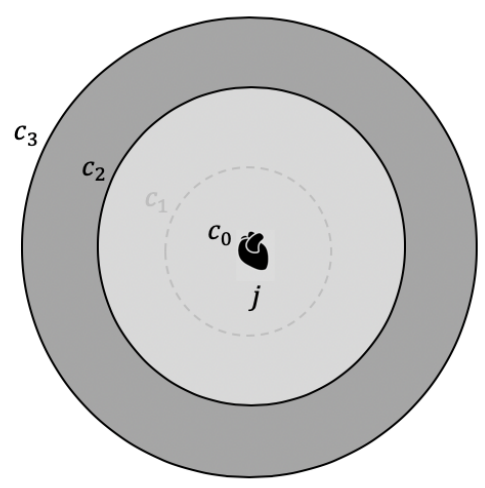

(c)

Figure 24. Possible 2 zones drawn from 3 concentric circles around donor hospital $j$.

transplants has been associated with supply to demand ratio disparity (Stewart et al., 2018), and it is a valid metric in measuring geographic disparity (Rana et al., 2015). Additionally, one study showed that supply to demand ratio is aligned with waiting time, that is, waiting time is lower in the transplant centers with a greater supply to demand ratio (Reese et al., 2014). We define access level as a function of supply to demand ratio, and of the distance between supply and demand locations. Our access level metric can be interpreted as transplant rate, since demand is the number of waitlist candidates and supply is the number of recovered hearts that have been transplanted.

In this section we explain how access level is defined. Given a proper zonal structure $\left\{z_{1 j}, z_{2 j}, \ldots, z_{\ell j}\right\}$ around each donor hospital $j \in H$, we quantify the access level $A_{t}$ of a given transplant center $t \in T$ as a function of the ratio of the total available supply for $t$ and the total demand at $t$. Let $s_{j}$ denote the supply at donor hospital $j$, and $d_{t}$ denote the demand at transplant center $t$. The set $Z=\{1,2, \ldots, \ell\}$ denotes the index set of the zones in the zonal structure. We use notation $T(h, j) \subseteq T$ to denote the set of transplant centers in zone $h$ around donor hospital $j$. We consider the supply $s_{j}$ to be available for transplant center $t$, if $t$ is, included in one of the zones assigned to the donor hospital $j$, that is, if there exists $h \in Z$ such that $t \in T(h, j)$ : 
DEFINITION 5 A donor hospital $j$ is associated with a transplant center $t$, if one of its zones contains $t$, i.e., there exists $h \in Z$ such that $t \in T(h, j)$.

DEFINITION 6 The set of donor hospitals associated with a given transplant center $t$ is denoted by $H(t)$, i.e., $H(t)=\{j \in H: \exists h \in Z$ s.t. $t \in T(h, j)\}$.

DEFINITION 7 The available supply for a transplant center $t$ is the sum of the supplies of all the donor hospitals associated with $t$, i.e., $\sum_{j \in H(t)} s_{j}$.

Let us consider the simple example in Figure 25 with two donor hospitals $\left\{j_{1}, j_{2}\right\}$, three transplant centers $\left\{t_{1}, t_{2}, t_{3}\right\}$, and two zones around each donor hospital. Transplant center $t_{1}$ is located in the first zone of donor hospital $j_{1}$, and the second zone around donor hospital $j_{2}$. Transplant center $t_{2}$ is located in the second zone of donor hospital $j_{1}$, and transplant center $t_{3}$ is in the first zone of donor hospital $j_{2}$. Hence, $H\left(t_{1}\right)=\left\{j_{1}, j_{2}\right\}$, and the available supply for $t_{1}$ is $s_{j_{1}}+s_{j_{2}} ; H\left(t_{2}\right)=\left\{j_{1}\right\}$, and the available supply for $t_{2}$ is $s_{j_{1}}$; and, finally, $H\left(t_{3}\right)=\left\{j_{2}\right\}$, and the available supply for $t_{2}$ is $s_{j_{2}}$.

Note that, since the zonal structure of a given donor hospital may contain multiple transplant centers, the supply in the donor hospital should be shared across transplant centers. Additionally, since distance between a transplant center and a donor hospital plays an important role in the heart allocation system (i.e., lower the distance, the better the access (Axelrod et al., 2010)), then, for defining the access level of each transplant center, two important factors should be considered: (i) the distance between a transplant center and the associated donor hospitals and (ii) the shared portion of the available supply, which depends on the number of transplant centers with which the supply of a given donor hospital needs to be shared and the corresponding level of demand (a transplant center with a greater demand is allocated a greater portion of supply).

To account for these two factors, we assign a weight $w_{h}$ to each zone $h$ around donor hospital $j$, such that inner zones have a greater weight (i.e., $w_{h} \geq w_{k}$, for 
$h<k)$, and we consider the demand for transplant centers which are associated with that donor hospital. Going back to the example in Figure 25, supply $s_{j_{1}}$ is shared between $t_{1}$ and $t_{2}$, with $t_{1}$ belonging to the first zone and $t_{2}$ belonging to the second zone around $j_{1}$. Hence, the shared portion of supply $s_{j_{1}}$ that goes to transplant center $t_{1}$ and $t_{2}$ is $\left(\frac{d_{t_{1}} w_{1}}{d_{t_{1} w_{1}+d_{t_{2}} w_{2}}} s_{j_{1}}\right)$ and $\left(\frac{d_{t_{2} w_{2}}}{d_{t_{1}} w_{1}+d_{t_{2}} w_{2}} s_{j_{1}}\right)$, respectively. Similarly, the shared portion of supply $s_{j_{2}}$ that goes to transplant center $t_{1}$ (in the second zone) and $t_{3}$ (in the first zone) is $\left(\frac{d_{t_{1} w_{2}}}{d_{t_{3}} w_{1}+d_{t_{1} w_{2}}} s_{j_{2}}\right)$ and $\left(\frac{d_{t_{3} w_{2}}}{d_{t_{3}} w_{1}+d_{t_{1} w_{2}}} s_{j_{2}}\right)$, respectively.

The access level $A_{t}$ for each transplant center $t$ is then computed as the ratio between the total supply going to transplant center $t$ and its demand $d_{t}$. In our example, the access level for transplant center $t_{1}$ is:

$$
A_{t_{1}}=\frac{1}{d_{t_{1}}}\left[\left(\frac{w_{1} d_{t_{1}}}{w_{1} d_{t_{1}}+w_{2} d_{t_{2}}} s_{j_{1}}\right)+\left(\frac{w_{2} d_{t_{1}}}{w_{2} d_{t_{1}}+w_{1} d_{t_{3}}} s_{j_{2}}\right)\right]
$$

the access level for $t_{2}$ is:

$$
A_{t_{2}}=\frac{1}{d_{t_{2}}}\left[\left(\frac{w_{2} d_{t_{2}}}{w_{1} d_{t_{1}}+w_{2} d_{t_{2}}} s_{j_{1}}\right)\right]
$$

the access level for $t_{3}$ is:

$$
A_{t_{3}}=\frac{1}{d_{t_{3}}}\left[\left(\frac{w_{1} d_{t_{3}}}{w_{2} d_{t_{1}}++w_{1} d_{t_{3}}} s_{j_{2}}\right)\right]
$$

Given a proper zonal structure $\left\{z_{1 j}, z_{2 j}, \ldots, z_{\ell j}\right\}$ defined for each donor hospital $j \in H$, and given the weights $w_{h}$ associated with each zone $h \in Z$, the shared supply and the access level $A_{t}$ for a transplant center $t$ are formally defined as follows:

DEFINITION 8 The shared supply $s_{j t}^{a}$ that is sent from donor hospital $j$ to transplant center $t$ located in zone $k$ of donor hospital $j$ is

$$
s_{j t}^{a}=\frac{d_{t} w_{k}}{\sum_{h \in Z} w_{h} \sum_{t^{\prime} \in T(h, j)} d_{t^{\prime}}} s_{j}
$$

The access level $A_{t}$ for a transplant center $t$ is the sum of the shared supplies of the donor hospitals associated with $t$ : 


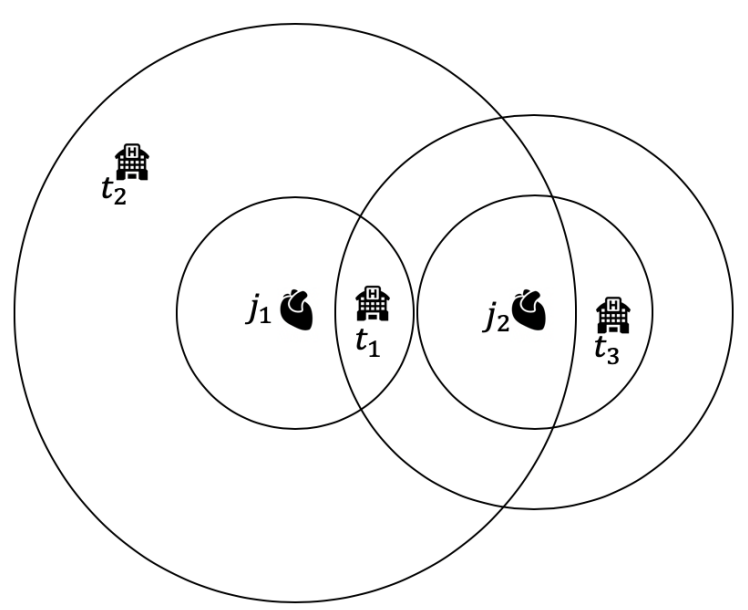

Figure 25. An example for clarifying access level computation for transplant centers.

DEFINITION 9 The access level $A_{t}$ for a transplant center $t$ is

$$
A_{t}=\frac{\sum_{j \in H(t)} s_{j t}^{a}}{d_{t}}
$$

\section{Access Level Disparity}

Variance, range, and other measures have been used in the literature for measuring geographic disparity (Marsh and Schilling, 1994; Barbati and Bruno, 2018). In this study, we measure access level disparity as the maximum difference from the average access level across transplant centers. Specifically, letting

$\bar{A}=\frac{\sum_{t \in T} A_{t}}{n}$ be the average access level across all the transplant centers, access level disparity is then measured as

$$
\operatorname{Max}_{t \in T}\left|A_{t}-\bar{A}\right|
$$

\section{The Boundary Allocation Problem}

Let $H$ be the set of $m$ donor hospitals and $T$ the set of $n$ transplant centers. For each donor hospital, $j \in H$, let $s_{j}$ be the amount of supply at donor hospital $j \in H$, and $d_{t}$ be the amount of demand at transplant center $t \in T$. Let $\mathcal{C}=\left\{c_{0}\right.$, $\left.c_{1}, \ldots, c_{q}\right\}$ be a set of $q$ predefined circles of increasing radii. Let $\ell$ be the number of 
zones to be defined around each donor hospital, and let $w_{h}$ be a weight assigned to zone $z_{h}, \forall h \in Z$. The access level for each transplant center $t \in T$ is $A_{t}$. The Boundary Allocation Problem looks for a zonal structure around each donor hospital such that access level disparity across the transplant centers is minimized.

\section{Model Formulation}

Let us define the binary parameter $a_{s k j t}, \forall s, k \in C, s<k, \forall j \in H, \forall t \in T$, which is equal to one if transplant center $t$ is located outside of circle $c_{s}$ and inside circle $c_{k}$ around donor hospital $j$, and zero otherwise. For a given zone $z_{h}, C_{h}^{i n} \subset C$ denotes the index set of possible inner circles, and $C_{h, s}^{o u t} \subset C$ denotes the index set of possible outer circles where $c_{s}$ is the inner circle. Note that, if $h=1$ (the first zone), then $C_{1}^{i n}=\{0\}$ and $C_{1,0}^{\text {out }}=\{1,2, \ldots, q-\ell+1\}$, whereas for $h>1$, $C_{h}^{\text {in }}=\{h-1, \ldots, q-\ell+h-1\}$ and $C_{h, s}^{\text {out }}=\{s+1, \ldots, q-\ell+h\}$.

We consider the set of binary decision variables $y_{s k h j}$, $\forall s \in C_{h}^{i n}, \forall k \in C_{h, s}^{\text {out }}, \forall h \in Z, \forall j \in H$, whose value is equal to one if $z_{h j}=\left(c_{s}, c_{k}\right)$, that is, if the $h$-th zone of donor hospital $j$ is defined as the pair of circles $\left(c_{s}, c_{k}\right)$, and it is equal to zero otherwise. For clarity of the mathematical model, we also consider the following auxiliary variables: the binary decision variables $v_{h j t}$, $\forall h \in Z, j \in H, t \in T$, whose value is equal to one if transplant center $t$ is located in zone $h$ around donor hospital $j$ and zero otherwise, and the continuous decision variables $A_{t}, \forall t \in T$ which indicate the access level for transplant center $t$. The 
mathematical model is as follows:

$$
\operatorname{Min} \operatorname{Max}_{t \in T}\left|A_{t}-\frac{\sum_{t \in T} A_{t}}{n}\right|
$$

subject to

$$
\begin{aligned}
& \sum_{t \in T} v_{h j t} \geq 1, \quad \forall j \in H, \quad \forall h \in Z \\
& \sum_{h \in Z} \sum_{j \in H} v_{h j t} \geq 1, \quad \forall t \in T \\
& \sum_{h \in Z} v_{h j t} \leq 1, \quad \forall j \in H, \quad \forall t \in T \\
& \sum_{h \in Z} \sum_{s \in C_{h}^{\text {in }}} \sum_{k \in C_{s, h}^{\text {out }}} y_{s k h j}=l, \quad \forall j \in H \\
& y_{f s h-1 j} \leq \sum_{k \in C_{s, h}^{\text {out }}} y_{s k h j}, \quad \forall f \in C_{h-1}^{\text {in }}, \forall s \in C_{f, h-1}^{\text {out }}, \forall j \in H \\
& v_{h j t}=\sum_{s \in C_{h}^{\text {in }}} \sum_{k \in C_{s, h}^{\text {out }}} a_{s k h t} y_{s k h j}, \quad \forall h \in Z, \quad \forall j \in H, \quad \forall t \in T \\
& A_{t}=\sum_{j \in H} \frac{\sum_{h \in Z^{\prime}} w_{h} v_{h j t}}{\sum_{h \in Z} \sum_{t^{\prime} \in T} w_{h} d_{t^{\prime}} v_{h j t^{\prime}}} s_{j}, \quad \forall t \in T \\
& y_{s k h j} \in\{0,1\}, \quad \forall h \in Z, \forall s \in C_{h}^{\text {in }}, \quad \forall k \in C_{s, h}^{\text {out }}, \quad \forall j \in H
\end{aligned}
$$

The objective function (2) minimizes access level disparity across the transplant centers. Constraints (3) ensure that each zone around each donor hospital contains at least one transplant center. Constraints (4) ensure that each transplant center is covered by at least one donor hospital (i.e., there exists at least one donor hospital zone to which the transplant center belongs). Constraints (5) ensure that all donor hospital and transplant center pairs are connected through no more than one of the zones around the donor hospital. Constraints (6) ensure that exactly $l$ zones are defined for each donor hospital. Constraints (7) ensure that the zonal structure around each donor hospital is properly defined. Constraints (8) and (9) define variables $v_{h j t}$ and $A_{t}$.

Note that the definition of access level $A_{t}$ (constraints (9)) introduces non-linearity in the model, which makes it difficult to solve even with standard 
available solvers. Moreover, model (2)-(10) is a general formulation which considers the same set of predefined circles $\mathcal{C}=\left\{c_{0}, c_{1}, \ldots, c_{q}\right\}$ around each donor hospital and defines the same number $\ell$ of zones around each donor hospital. The formulation can be generalized to consider a different number $\ell_{j}$ of zones for each donor hospital and a different set $\mathcal{C}_{j}$ of circles around each donor hospital. However, current practice assumes the same number of zones around each donor hospital; hence, it would be unrealistic to assume otherwise. Additionally, it would be unrealistic to assume the number of zones to be greater than five (the current policy considers five zones - Local trough Zone D - and one additional zone - Zone E - as the national level). In particular, as will be shown in section $\mathrm{D}$, the results of our data-driven strategy to compute the weights associated with each zone assign virtually all the weight to the first three zones, i.e., $z_{1}, z_{2}$ and $z_{3}$, with the other zones getting nearly zero weight. For these reasons, in our computational experiments we analyzed two scenarios which arise when the number of zones around each donor hospital is equal to either two or three, $\ell=2$ or $\ell=3$. For these two cases, the model formulation for BAP can be simplified. We showed the formulation for $\ell=3$ in the Appendix A, and the formulation for $\ell=2$ can be defined similarly.

\section{Weight Tuning}

As explained in section 2, the weights $w_{h}, \forall h \in Z$ assigned to each zone, indicate the priority that the zones receive in the allocation process. With the current allocation process, a recovered organ is first offered to candidates registered in transplant centers closer to the donor hospital (i.e., transplant centers that belong to the first zone around the donor hospital), and then, if a match is not found, it is offered to candidates registered in transplant centers that belong to the zones further away from the donor hospital. Hence, the role of the weights in the mathematical model is paramount in distributing the available organs effectively once the zonal structure is implemented. 
We applied a data-driven approach to define the weights such that the difference between the potential supply (i.e., the available supply for a transplant center) and the realized supply (i.e., the amount of organs effectively provided to a transplant center) is as low as possible. Specifically, we define $P_{h t}$ as the total potential supply from all the donor hospitals that are associated with transplant center $t \in T$ through their zone $h$. Similarly, we define $R_{h t}$ as the total realized supply from all the donor hospitals that are associated with transplant center $t \in T$ through their zone $h$.

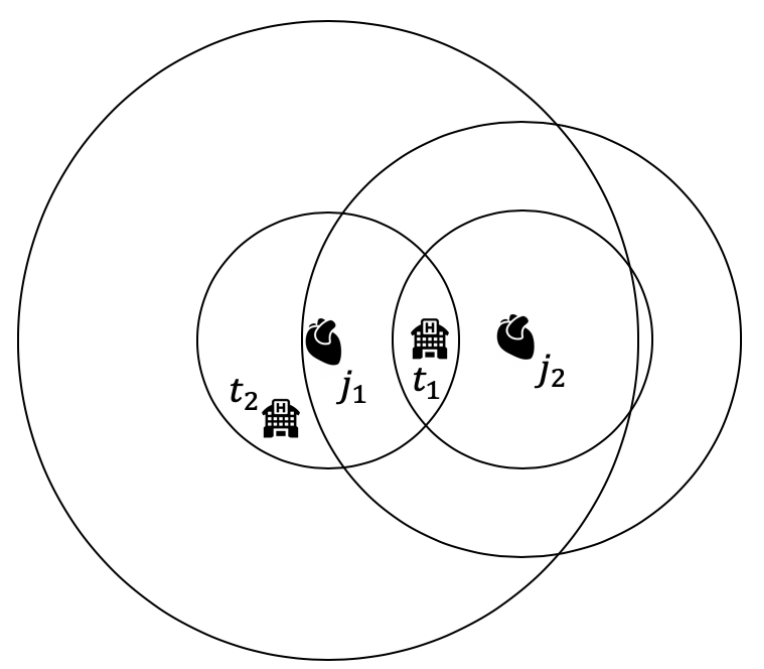

Figure 26. An example to illustrate the calculation for potential and realized supply

Consider Figure 26 with two transplant centers, $t_{1}$ and $t_{2}$, with demand $d_{t_{1}}$ and $d_{t_{2}}$, respectively, and two donor hospitals, $j_{1}$ and $j_{2}$, with supply $s_{j_{1}}$ and $s_{j_{2}}$, respectively, each with a zonal structure consisting of two zones. For transplant center $t_{1}$, which is located in the first zone around both donor hospitals, the potential supply from the first zone is $P_{1 t_{1}}=\frac{d_{t_{1}}}{d_{t_{1}}+d_{t_{2}}} s_{j_{1}}+s_{j_{2}}$, while for transplant center $t_{2}$ it is $P_{1 t_{2}}=\frac{d_{t_{2}}}{d_{t_{1}}+d_{t_{2}}} s_{j_{1}}$. The supply in $j_{1}$ is divided between the two transplant centers $t_{1}$ and $t_{2}$ as a portion of their demand. For calculating $R_{h t}$, we define $v_{j t}$ as the number of organs sent and transplanted from donor hospital $j$ to transplant center $t$; consequently, in this scenario $R_{1 t_{1}}=v_{j_{1} t_{1}}+v_{j_{2} t_{1}}$, and $R_{1 t_{2}}=v_{j_{2} t_{2}}$. 
In our approach we considered the current homogeneous 6-Zone structure for computing the potential and the realized supply. Specifically, we used the Thoracic Simulation Allocation Model (TSAM) to calculate $v_{j t}$. TSAM is a simulation software developed by the US Scientific Registry of Transplant Recipients (SRTR) (SAM, 2017) to simulate the allocation of hearts to candidates waiting for heart transplants based on an event-sequenced Monte Carlo technique. We used TSAM input data, from July $1^{\text {st }}, 2009$ to June $30^{\text {th }}$, 2011. The data contains 8,593 adult candidates (i.e., age $\geq 18$ ). Of these transplant candidates, 2,621 of them were registered at the beginning of the study period, and 5,972 new candidates were added to the transplant list during the study period. 159 of the patients required combined heart-lung transplant and were excluded from the study. The final data set included a total of 8,434 heart transplant candidates and 3,770 donated heart organs.

We applied the following optimization model to determine the optimal weights to minimize the difference between the potential supply and the realized supply:

$$
\begin{aligned}
& \operatorname{Min} \sum_{h \in Z} \sum_{t \in T}\left|P_{h t} w_{h}-R_{h t}\right| \\
& \text { subject to } \\
& \sum_{h \in Z} w_{h}=1, \\
& w_{h} \leq w_{k}, \quad \forall h, k \in Z(h>k) \\
& w_{h} \geq 0 . \quad \forall h \in Z
\end{aligned}
$$

Objective function (11) minimizes the total absolute difference between the potential and realized supply across all transplant centers. Constraint (12) normalizes the weights, and constraints (13) ensure that inner zones have greater weights.

The results of the model are shown in Table 17. The weight for the first zone $z_{1}$ was significantly higher than the weights for the other zones. This was an 
expected result given that the simulation results from TSAM showed that more than $85 \%$ of the organs are allocated to the first zone. We used the weights in Table 17 for our model to design a heterogeneous zonal structure around donor hospitals.

\section{TABLE 17}

Zonal potential supply, zonal realized supply, and zonal weight based on the current homogeneous zonal structure

\begin{tabular}{lccccc}
\hline & $\begin{array}{c}z_{1} \\
(\text { Zone A })\end{array}$ & $\begin{array}{c}z_{2} \\
(\text { Zone B })\end{array}$ & $\begin{array}{c}z_{3} \\
(\text { Zone C) }\end{array}$ & $\begin{array}{c}z_{4} \\
(\text { Zone D) }\end{array}$ & $\begin{array}{c}z_{5} \\
(\text { Zone E) }\end{array}$ \\
\hline$\sum_{h \in Z} \sum_{t \in T} P_{h t}$ & 5,057 & 575 & 81 & 15 & 0 \\
\hline$\sum_{h \in Z} \sum_{t \in T} R_{h t}$ & 3,421 & 377 & 51 & 7 & 0 \\
\hline$w_{h}$ & 0.90 & 0.07 & 0.03 & 0.00 & 0.00 \\
\hline
\end{tabular}

\section{E Experimental Analysis}

\section{Data}

We used the data provided by OPTN on transplanted heart organs and waitlist heart candidates from 2011 to 2014. We focused on donor hospitals and transplant centers in the continental United States; hence, we excluded Puerto Rico, Hawaii, and Alaska from our analysis. Supply in each donor hospital was the number of recovered hearts after excluding the discarded hearts. Demand in each transplant center was the number of transplant candidates registered on the heart transplant list on a given year, excluding the waiting list candidates prior to that year. We excluded pediatric patients (i.e., age $<18$ ), inactive patients ${ }^{1}$, and patients on a combined transplant list (e.g., heart-lung). Table 18 shows the number of donor hospitals, total supply, number of transplant centers, and total demand for the four year period. We used data from 2014 to determine the optimal

\footnotetext{
${ }^{1}$ Inactive is the designation for patients who are temporarily removed from the heart transplant waiting list due to health conditions or personal circumstances.
} 
heterogeneous zonal structure by solving our BAP model, while we used data from 2011, 2012, and 2013 to validate the model.

\section{TABLE 18}

The demographic of the study population

\begin{tabular}{lcccc}
\hline & 2011 & 2012 & 2013 & 2014 \\
\hline Number of Donor Hospitals & 436 & 437 & 453 & 698 \\
Total Supply & 1,606 & 1,665 & 1,767 & 2,125 \\
\hline Number of Transplant & 104 & 107 & 107 & 115 \\
Centers & & & & \\
Total Demand & 2,832 & 3,067 & 3,333 & 3,726 \\
\hline
\end{tabular}

\section{Scenario Description}

The results from our weight tuning strategy revealed the high importance of the first three zones in the current heart allocation system. Thus, we used our BAP model to determine an optimal heterogeneous zonal structure, with two zones $(\ell=2)$ and three zones $(\ell=3)$ around each donor hospital, and we refer to these scenarios as heterogeneous 2-Zone and 3-Zone structures. Zonal structures around each donor hospital were defined by selecting the best circles from a predefined set. The radius for the predefined circles ranged from 100 to 1,200 miles in increments of 100 (for a total of 12 predefined circles). For designing the heterogeneous 2-Zone structure we considered $w_{1}=0.9$ and $w_{2}=0.1$, and for the heterogeneous 3-Zone structure we considered $w_{1}=0.9, w_{2}=0.07$, and $w_{3}=0.03$. We compared the results of our model with those of the corresponding homogeneous zonal structures which we refer to as homogeneous 2-Zone and 3-Zone structures. The 2-Zone homogeneous structure contained two zones around each donor hospital, with the first and second zones having radii of 500 and 1000 miles, respectively. The homogeneous 3-Zone structures contained three zones, with the first, second, and third zones having radii of 500, 1000, and 1500 miles, respectively. 
The comparison between homogeneous and heterogeneous zonal structures is carried out considering the following metrics:

1. Access Level Disparity: The access level disparity described in Section 3 was compared for each zonal structure. In addition, we measured the range and median access level across the transplant centers for both homogeneous and heterogeneous zonal structures.

2. Zonal Structure Heterogeneity: For each circle $c_{k}$ in $\mathcal{C}$, we calculated the percentage of donor hospitals that contain circle $c_{k}$ in their zonal structure.

3. Distance Traveled by Organs: For each transplant center, we measured the distance that an organ has to travel to be transplanted in that transplant center. For example, if for transplant center $t$, five organs are sent from a donor hospital 100 miles away, and 10 organs are sent from a donor hospital 40 miles away, the weighted average distance for an organ to travel to transplant center $t$ is: $\frac{5 \times 100+10 \times 40}{5+10}=60$ miles. We measured the variation in travel distance across transplant centers. Additionally, we calculated the national average distance traveled by organs.

The robustness of the model with respect to changes in the input data was evaluated using an extensive sensitivity analysis. Specifically, we considered the following analyses:

1. Supply and Demand: We investigated whether our heterogeneous zonal structure is robust to changes in supply and demand by using the data from 2014 as the training data set and the data from 2011 to 2013 as the test data sets. We solved the BAP model with the training data set to obtain an optimized heterogeneous zonal structure. Then, we compared the access level across transplant centers using test data set and the optimized heterogeneous zonal structure. 
2. Maximum Circle Radius: Maximum circle radius is the radius of the outermost circle in the predefined set of concentric circles. We ran the model multiple times by changing the maximum circle radius from 700 to 1,500 miles in increments of 100. We analyzed the effect of the changes on the access level disparity and on the travel distance.

\section{F Results}

\section{Access Level Disparity}

Access level disparity for heterogeneous 2-Zone structure was 0.003 , while for homogeneous 2-Zone structure it was 0.307. Additionally, access level across transplant centers for heterogeneous 2-Zone structure ranged from 0.56 to 0.57 hearts per candidate (median=0.57), and for homogeneous 2-Zone structure ranged from 0.26 to 0.80 hearts per candidate (median=0.59). We calculated the average access level across the transplant centers in each OPO. Figure 27 shows the variation in the average access level across OPOs. OPOs with light color do not contain any transplant center. For heterogeneous 2-Zone structure the access level ranged from 0.56 to 0.57 hearts per candidate across OPOs, while for homogeneous 2-Zone structure it ranged from 0.26 to 0.80 hearts per candidate.

Access level disparity for heterogeneous 3-Zone structure was 0.009, and for homogeneous 3-Zone structure was 0.183. Specifically, the access level across transplant centers for heterogeneous 3-Zone structure ranged from 0.56 to 0.58 hearts per candidate (median=0.57), and for homogeneous 3-Zone structure ranged from 0.38 to 0.73 hearts per candidate (median=0.57). Figure 28 shows the average access level for both heterogeneous and homogeneous 3-Zone structures across OPOs. For heterogeneous 3-Zone structure the access level ranged from 0.56 to 0.58 hearts per candidate across OPOs, while for homogeneous 3-Zone structure it ranged from 0.39 to 0.72 hearts per candidate across OPOs. 

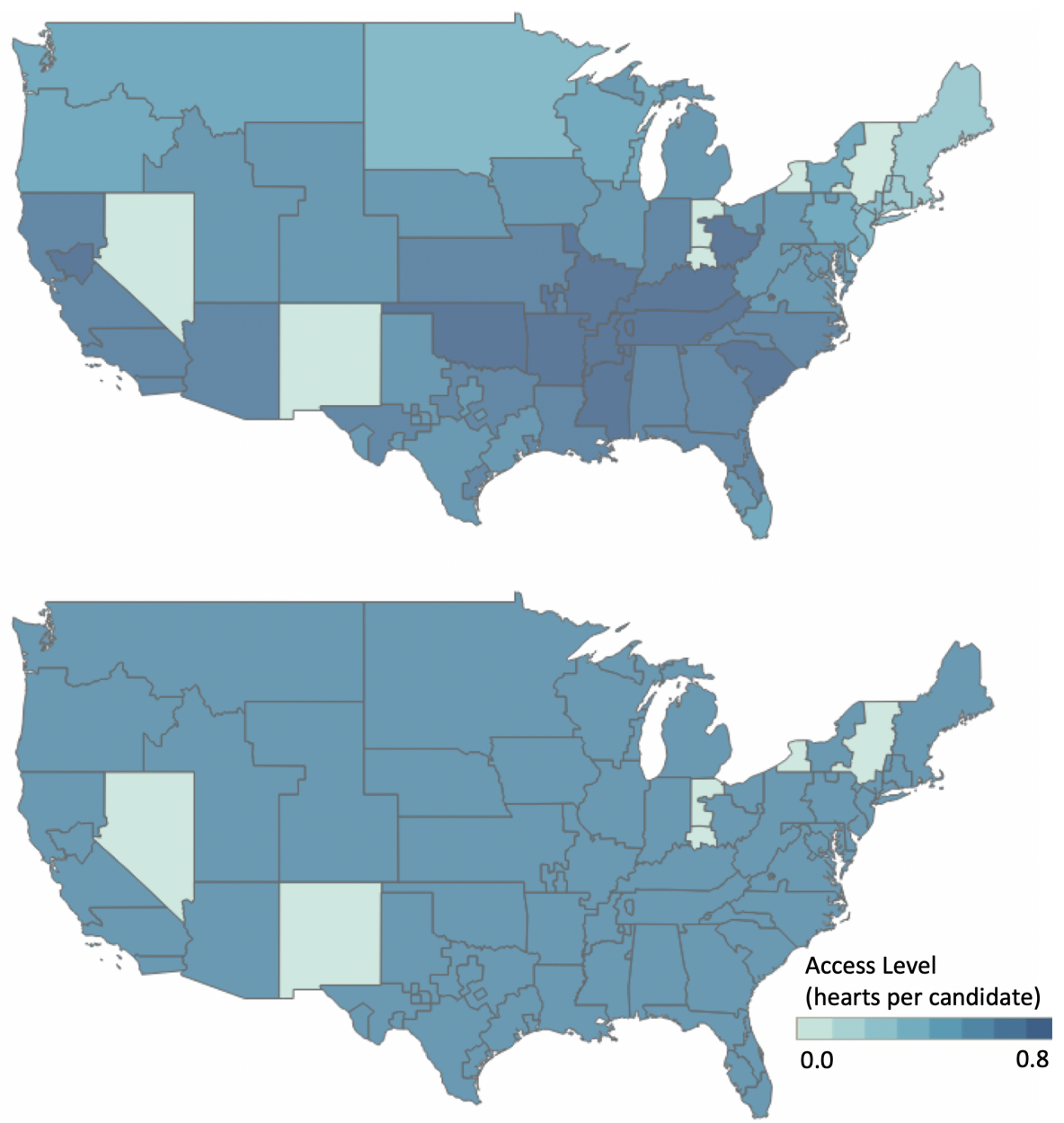

Figure 27. Variation in access level across OPOs for homogeneous 2-Zone structure (top), and heterogeneous 2-Zone structure (bottom).

\section{Zonal Structure Heterogeneity}

Figures 29 and 30 show the percentage of donor hospitals for different zone radius for heterogeneous 2 -Zone and 3-Zone structures, respectively. We referred to the first zone as Zone A, the second zone as Zone B, and the third zone as Zone C. Note that for the homogeneous 2-Zone structure for $100 \%$ of the donor hospitals, Zone A radius was 500, and Zone B radius was 1,000 miles. For the heterogeneous 2-Zone structure, for $57 \%$ of the donor hospitals Zone A radius was less than or equal to 500 miles, and for $56 \%$ of the donor hospitals Zone B radius was less than 

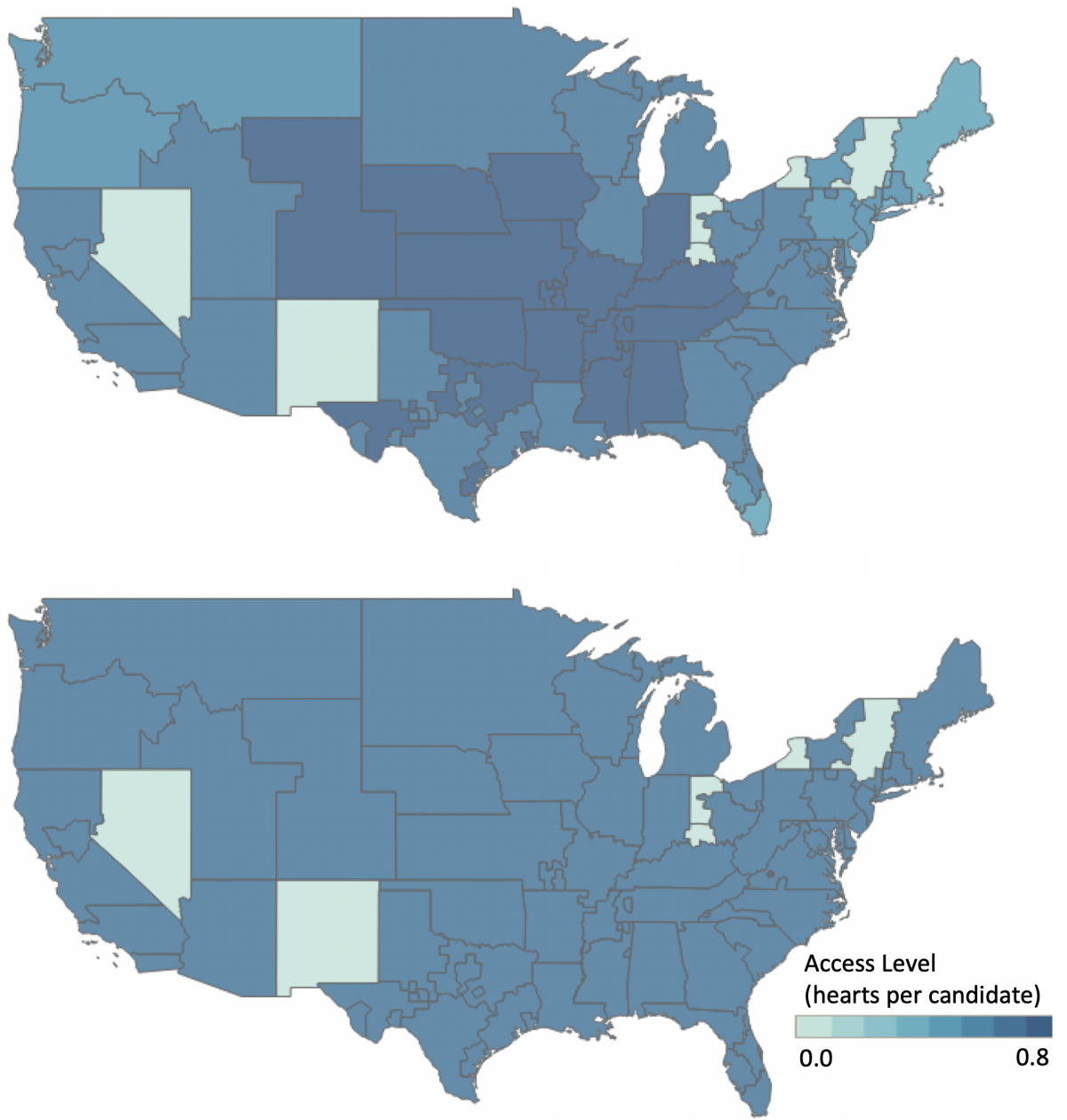

Figure 28. Variation in access level across OPOs for homogeneous 3-Zone structure (top), and heterogeneous 3-Zone structure (bottom).

or equal to 1,000 miles.

Note that with 12 predefined circles there are 66 possibles configurations for a 2-Zone structure. The optimal heterogeneous 2-Zone structure assigned all the 66 possible configurations to the 698 donor hospitals.

The homogeneous 3-Zone structure is such that for $100 \%$ of the donor hospitals Zone A radius was 500, Zone B radius was 1,000, and Zone $\mathrm{C}$ radius was 1,500 miles. For the heterogeneous 3-Zone structure, for $48 \%$ of donor hospitals Zone A radius was less than or equal to 500 miles; for $71 \%$ of the donor hospitals 


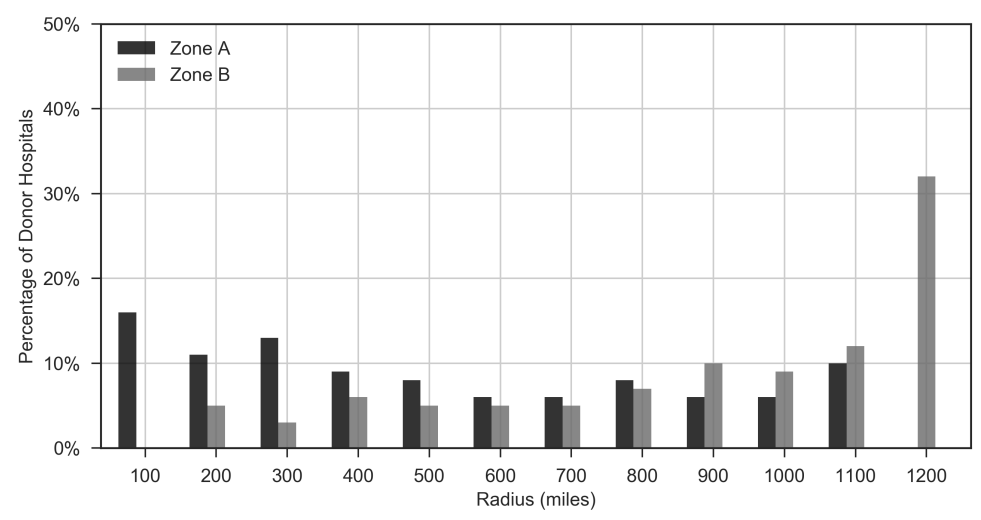

Figure 29. Zone A and Zone B radius around donor hospitals for heterogeneous 2-Zone structure

Zone B radius was less than or equal to 1,000 miles, and for $100 \%$ of the donor hospitals Zone $\mathrm{C}$ radius was less than or equal to 1,200 miles.

Additionally, based on the 12 predefined set of circles, there are 222 possible configurations for a 3-Zone structure. Among them the model assigned only 126 different configurations to the 698 donor hospitals.

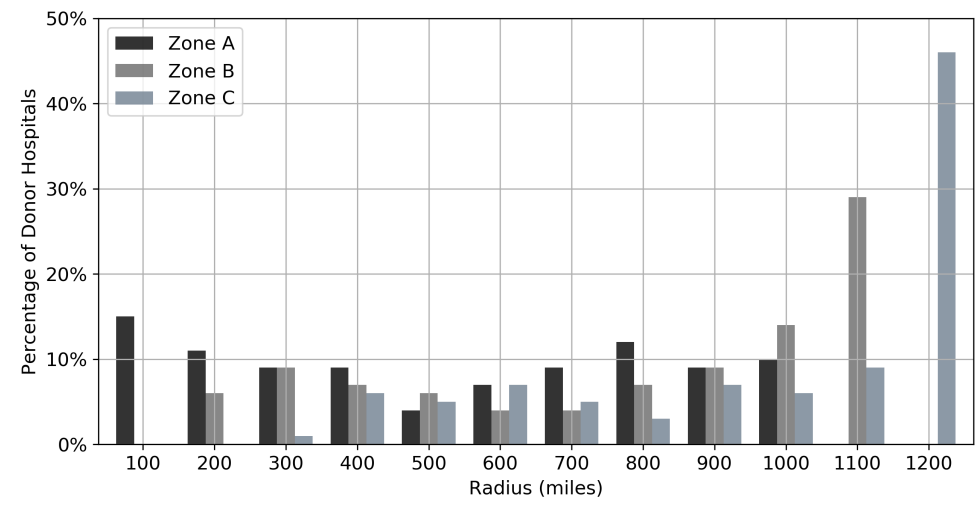

Figure 30. Zone A, Zone $\mathrm{B}$ and Zone $\mathrm{C}$ radius around donor hospitals for heterogeneous 3-Zone structure

\section{Distance Traveled by Organs}

Table 19 shows the minimum, maximum, median, and national average distance traveled by organs in homogeneous and heterogeneous zonal structures. 
The average distance traveled by organs for a heterogeneous 2-Zone structure ranged from 243 to 634 miles across transplant centers, and for a homogeneous 2-Zone structure, it ranged from 240 to 460 miles across transplant centers. The average distance traveled by organs for heterogeneous 3-Zone structure, ranged from 230 to 595 miles across transplant centers, and for homogeneous 3-Zone structure, it ranged from 267 to 514 miles across transplant centers.

\section{TABLE 19}

Minimum, maximum, median, and average distance traveled by organs (miles) across transplant centers

\begin{tabular}{c|cc|cc}
\hline & \multicolumn{2}{|c|}{ Heterogeneous } & \multicolumn{2}{c}{ Homogeneous } \\
& 2-Zone & -Zone & 2-Zone & 3-Zone \\
\hline Minimum & 243 & 230 & 240 & 267 \\
Maximum & 634 & 595 & 460 & 514 \\
Median & 440 & 385 & 368 & 379 \\
National Average & 425 & 380 & 368 & 375 \\
\hline
\end{tabular}

\section{G Sensitivity Analysis}

\section{Supply and Demand}

To determine if the access level in both heterogeneous 3-Zone and 2-Zone structures was robust to changes in supply and demand, we validated the results from the BAP model with multiple test data sets. Figures 31 and 32 show the variation in access level across transplant centers for 2011 to 2014 for heterogeneous 2-Zone and 3-Zone structures, respectively.

Since the zonal structure is optimized using 2014 data, the variation in access level for this year is at the minimum level. We see from the figures that even with test data sets the variation in access level across transplant centers is lower with a heterogeneous zonal structure than with a homogeneous zonal structure.

It should be mentioned that the access level disparity for heterogeneous 
2-Zone structure was 0.51, 0.50, and 0.49 for the 2011, 2012, and 2013 data, respectively, while for the homogeneous zonal structure it was $0.51,0.51$, and 0.50 , respectively. Similarly for heterogeneous 3-Zone structure access level disparity was 0.56, 0.54, and 0.53 for the 2011, 2012, and 2013, respectively, while for homogeneous 3 -Zone structure was $0.57,0.54$, and 0.53 , respectively. It seems that the access level disparity did not change with heterogeneous zonal structure; however, the access level disparity minimizes the largest different from average access level across transplant centers, which can be sensitive to outliers (as shown in the box plots in Figure 31 and 32). However, Figures 31 and 32 show the variation in access level across transplant centers for heterogeneous zonal structure was significantly less than for the homogeneous zonal structure.

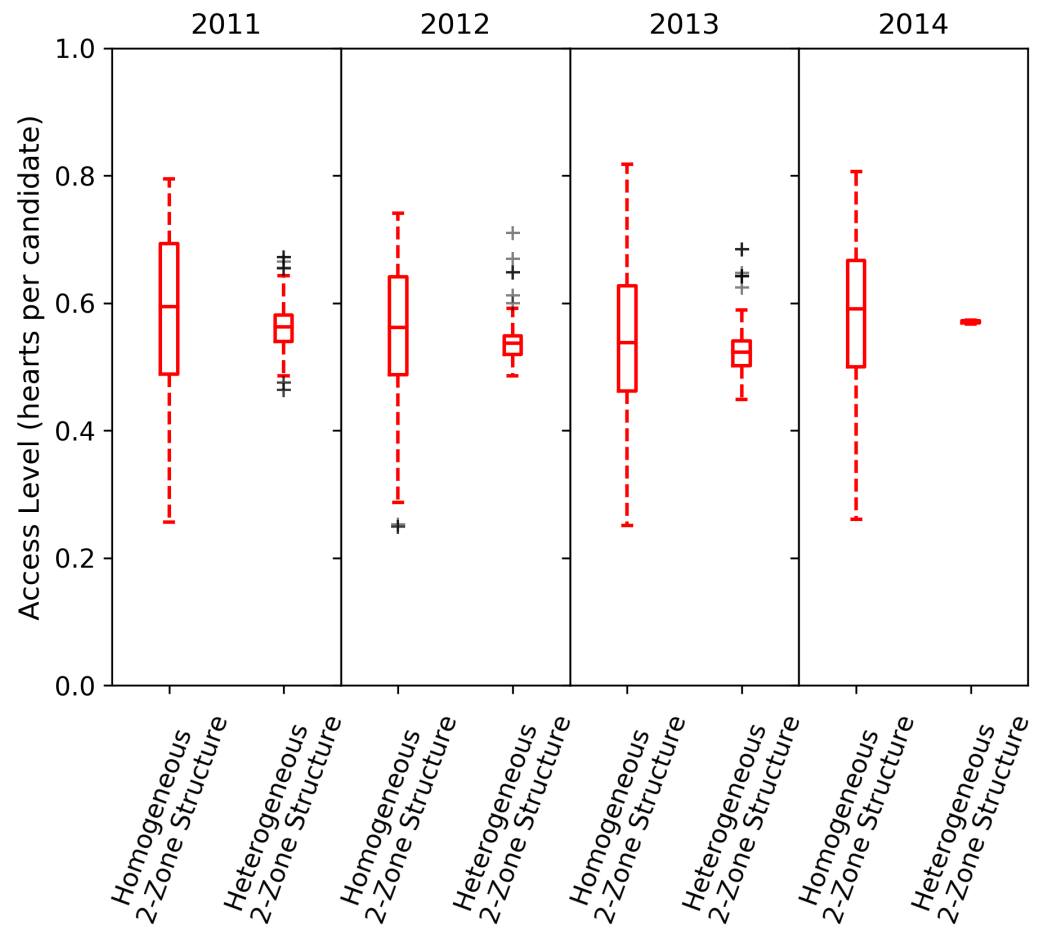

Figure 31. Variation in access level across transplant centers for heterogeneous and homogeneous 2-Zone structures with test (2011-2013) and training data set (2014). 


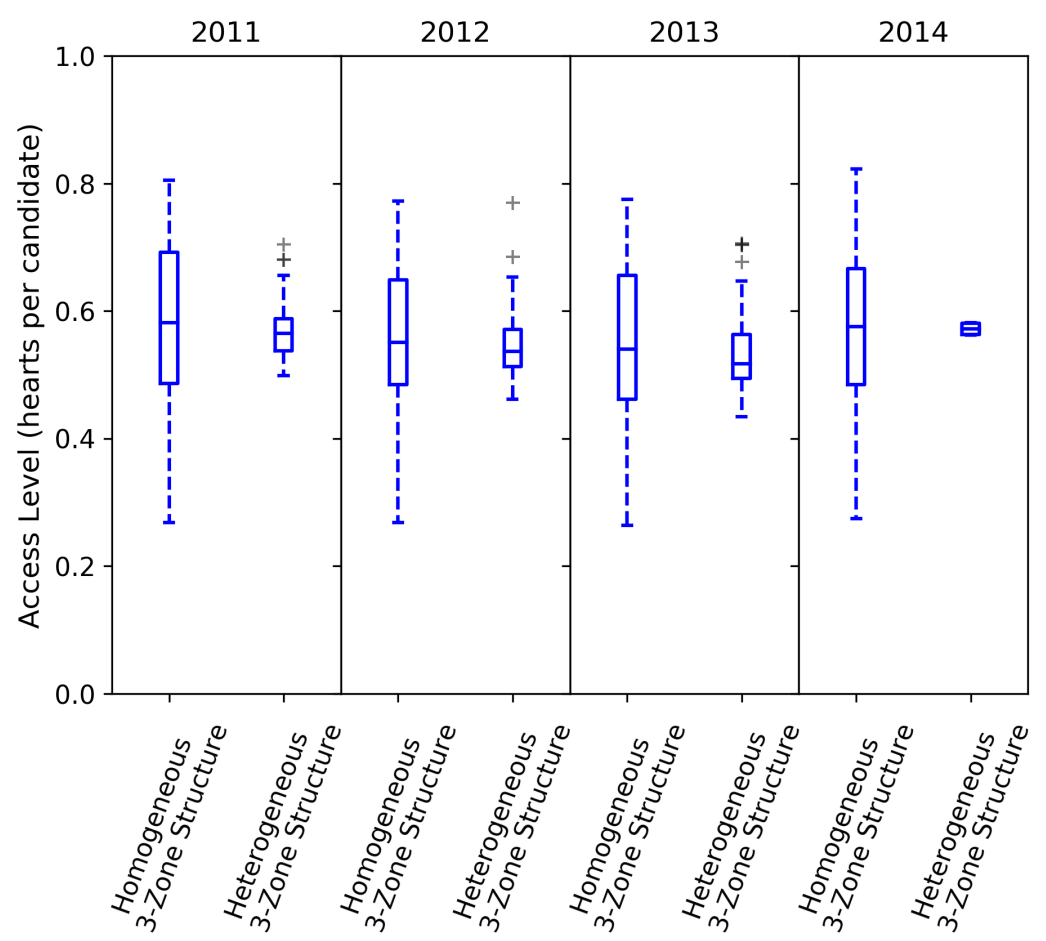

Figure 32. Variation in access level across transplant centers for heterogeneous and homogeneous 3-Zone structures with test (2011-2013) and training data set (2014).

\section{Maximum Circle Radius}

\subsection{Access Level Disparity}

By increasing the maximum circle radius from 700 miles to 1,500 miles, the access level disparity decreased from 0.135 to 0.004 for the heterogeneous 3-Zone structure and from 0.099 to 0.005 for the heterogeneous 2-Zone structure (Table 20).

Figure 33 shows the variation in access level across transplant centers for 2-Zone and 3-Zone structures. When the maximum circle radius was increased, the variation in access level across transplant centers for both heterogeneous 2-Zone and 3-Zone structures decreased. When the maximum circle radius was greater than or equal to 1,200 miles, the variation in access level did not change. When the maximum circle radius increased the median access level across transplant centers 
TABLE 20

Access level disparity for heterogeneous zonal structure with different values for maximum circle radius

\begin{tabular}{ccc}
\hline $\begin{array}{c}\text { Maximum } \\
\text { Radius (miles) }\end{array}$ & $\begin{array}{c}\text { 2-Zone } \\
\text { Structure }\end{array}$ & $\begin{array}{c}\text { 3-Zone } \\
\text { Structure }\end{array}$ \\
\hline 700 & 0.099 & 0.135 \\
800 & 0.059 & 0.093 \\
900 & 0.036 & 0.058 \\
1000 & 0.022 & 0.034 \\
1,100 & 0.014 & 0.020 \\
1,200 & 0.003 & 0.009 \\
1,300 & 0.003 & 0.006 \\
1,400 & 0.003 & 0.005 \\
1,500 & 0.005 & 0.004 \\
\hline
\end{tabular}

slightly increased from 0.55 to 0.59 hearts per candidate for heterogeneous 2-Zone structure, and from 0.54 to 0.57 hearts per candidate for heterogeneous 3-Zone structure.

\subsection{Distance Traveled by Organs}

Figure 34 shows the variation in the average distance traveled by organs across transplant centers. When the maximum circle radius increased the average travel distance for both the heterogeneous 2-Zone and 3-Zone structures slightly increased. When the maximum circle radius increased the variation in average travel distance across transplant centers did not change. We compared the national average travel distance for heterogeneous zonal structure versus homogeneous zonal structure (Figure 35). Red lines correspond to the 2-Zone structures and blue lines correspond to the 3-Zone structures. When the maximum circle radius increased the national average distance traveled by organs for both the heterogeneous 2-Zone and 3-Zone structures increased. However, when maximum circle radius was less than or 


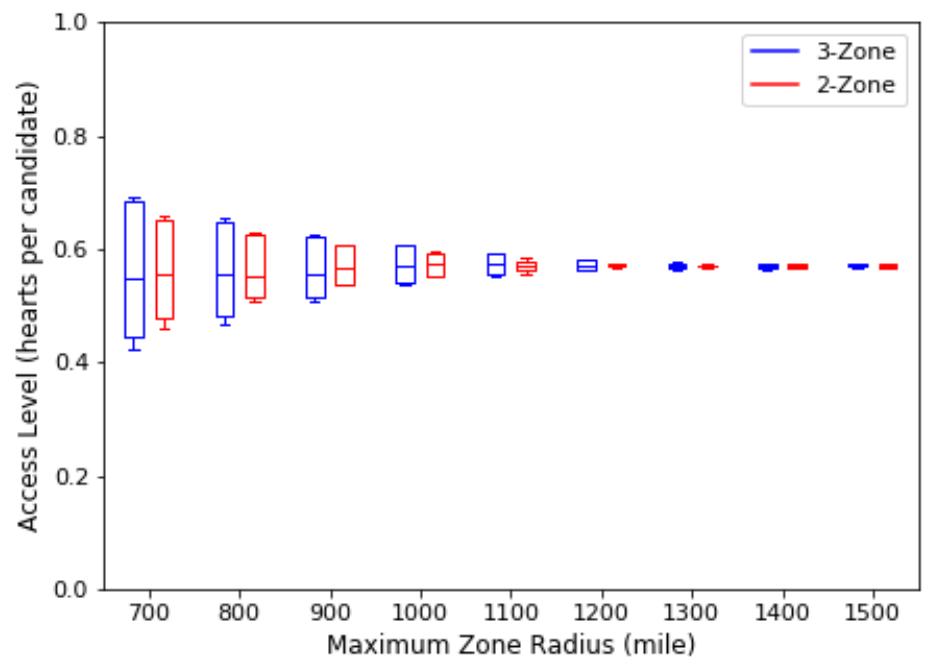

Figure 33. Variation in access level across transplant centers for heterogeneous zonal structure

equal to 900 miles, the average travel distance for the heterogeneous 2-Zone structure was less than for the homogeneous 2-Zone structure. On the other hand, when maximum circle radius was less than or equal to 1,200 miles, the national average distance for the heterogeneous 3-Zone structure was less than for the homogeneous 3-Zone structure.

\section{H Discussion}

Our optimization model for tuning the weight assigned to each zone showed that designing up to three zones around each donor hospital was sufficient to minimize access level disparity. Thus, we used BAP model and designed two different zonal structures, consisting of two zones and three zones.

Our results indicated that heterogeneous 2-Zone and 3-Zone structures reduced access level disparity significantly. Our analysis of the effect of maximum circle radius on access level disparity indicated that increasing maximum circle radius decreased access level disparity. However, no circle needed to be greater than 


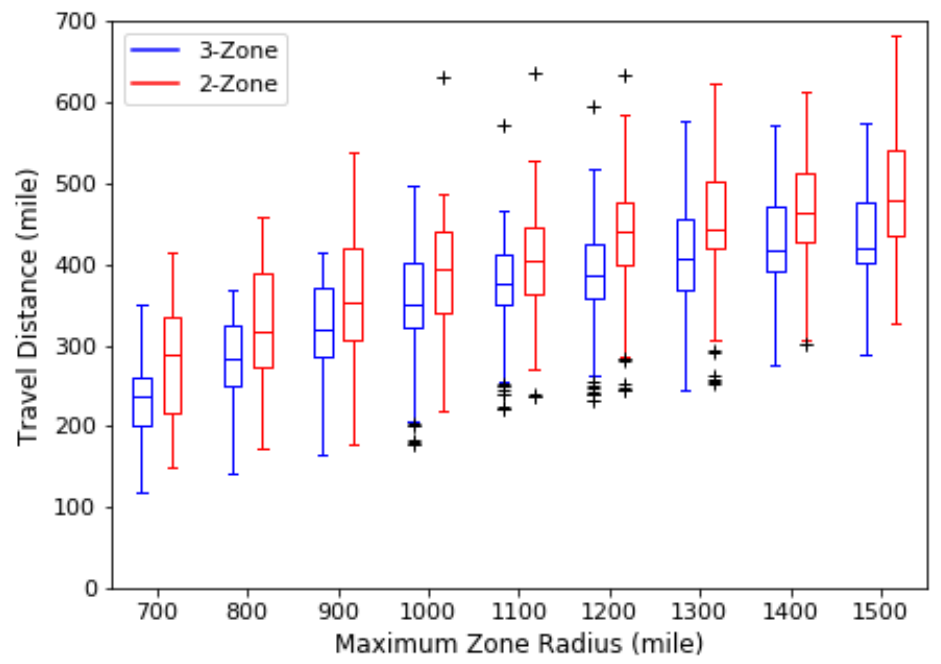

Figure 34. Variation in travel distance across transplant centers for heterogeneous zonal structure

1,200 miles to achieve minimum access level disparity for both heterogeneous 2-Zone and 3-Zone structures.

Figure 35 shows that, of any value for the maximum circle radius, the national average distance for heterogeneous 2-Zone structure was greater than for the heterogeneous 3-Zone structure. This comparison suggested that a heterogeneous zonal structure consisting of three zones had a lower national average travel distance and the access level disparity.

In summary, our results showed that the heterogeneous 3-Zone structure of maximum circle radius of 1,200 miles reduced access level disparity significantly and did not increase the national average distance traveled by organs. Additionally, with the heterogeneous 3-Zone structure of maximum circle radius of 1,200 miles, Zone A radius for $51 \%$ of donor hospitals was less than or equal to 500 miles, Zone B radius for $71 \%$ of donor hospitals was less than or equal to 1,000 miles and Zone $\mathrm{C}$ radius for all the donor hospitals was less than or equal to 1,200 miles. In contrast to homogeneous 3-Zone structure where organs might travel up to 1,500 miles, with heterogeneous 3-Zone structure organs might travel up to 1,200 miles. 


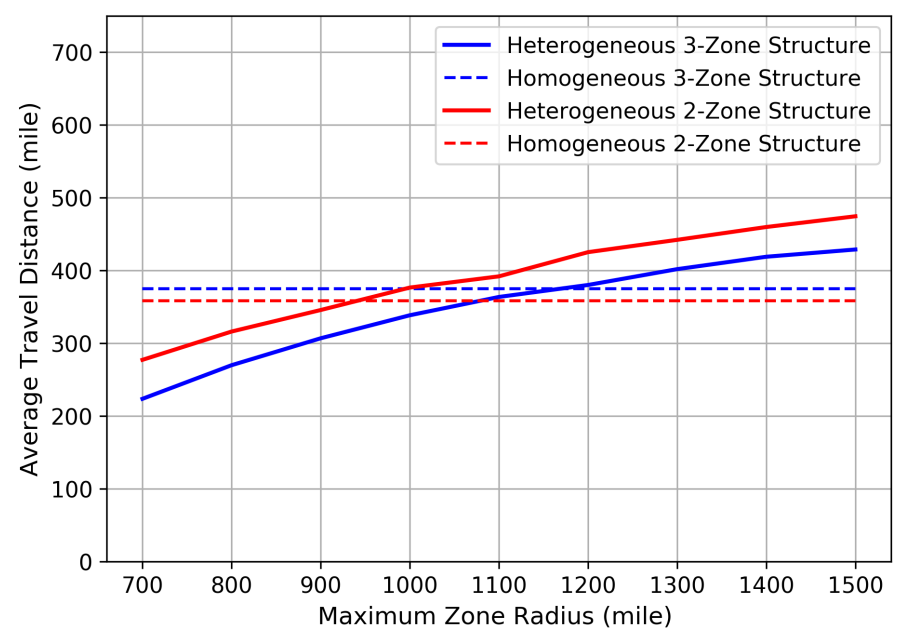

Figure 35. National average distance traveled by organs for heterogeneous zonal structure with different maximum zone radii in comparison with homogeneous zonal structure. The red lines are 2-Zone structures and the blue lines are 3-Zone structures

Furthermore, our sensitivity analysis on changes in supply and demand showed that heterogeneous zonal structure was robust to changes in supply and demand, meaning that with changes in supply and demand the access level disparity of that heterogeneous zonal structure was significantly less than for homogeneous zonal structure.

\section{Conclusion}

We introduced a new optimization problem, that is, Boundary Allocation Problem, to design a heterogeneous zonal structure around donor hospitals to reduce geographic disparity in access to heart transplants. To measure geographic disparity, we defined a new metric that is a function of supply to demand ratio and of the distance between supply and demand locations. Disparity in access to transplants was quantified as the largest difference between transplant centers' access level from the average access level across all the transplant centers.

We made some assumptions in our BAP model. First, we did not consider the medical status of the candidates in prioritizing them. In our model all 
candidates are equal regardless of their age, blood type, waiting time or other candidates characteristics. Second, we did not include any acceptance criteria to model candidates' behavior when an offer is made. Instead, we assumed all offers are accepted. Third, we did not consider the time varying nature of the allocation system. Currently, OPTN uses TSAM for evaluating different heart allocation system. The current version of TSAM is not compatible with heterogeneous zonal structure. Consequently, currently we cannot analyze the effect of our optimized heterogeneous zonal structure on transplant rate, waiting time and mortality rate. Developing a simulation model which is compatible with a heterogeneous zonal structure is objective of future research.

Additionally, our disparity metric for measuring geographic disparity can be sensitive to outliers since it minimizes the largest difference from the average access level across transplant centers. However, using such a metric to design an optimal heterogeneous zonal structure resulted in lowest variation in access level since the metric minimizes the gap between the possible outlier and the average access level.

We performed an extensive computational analysis to evaluate the benefit of heterogeneous zonal structure instead of the current homogeneous zonal structure. We concluded that in order to reduce geographic disparity, designing three zones around donor hospitals is sufficient. Our optimized heterogeneous zonal structure not only reduced geographic disparity in access to heart transplants without increasing average distance traveled by organs, but also was robust to changes in supply and demand. Additionally, with heterogeneous zonal structure no organ travels longer than 1,200 miles, while with homogeneous zonal structure some organs might travel up to 1,500 miles. Using mathematical modeling to design a zonal structure by taking into account the distribution of supply and demand across the country led to a better solution. 


\section{CHAPTER IV}

\section{HEART ALLOCATION SIMULATION MODEL}

\section{A Introduction}

An optimization model alone provides only a deterministic view of the impact of any policy for allocating organs. A simulation model provides more information about the uncertain environment of the organ allocation system. United Network of Organ Sharing (UNOS) currently uses the Thoracic Simulation Allocation Model (TSAM) to evaluate the impact of any heart allocation policy (Colvin et al., 2016). TSAM has the ability to simulate the allocation of hearts to candidates waiting for heart transplants based on an event-sequenced Monte Carlo technique. TSAM has been purposefully designed to serve studies investigating alternative organ allocation models (Colvin et al., 2016).

TSAM, which is available to the public but only as an executable file, offers very little flexibility to its users in evaluating different policies (TSAM, 2017). For instance, it does not support the heterogeneous zonal structure that we designed in Chapter III. The radius for the zones can be modified in TSAM, but the zonal structure for the donor hospitals must be the same. To overcome this limitation, we have developed a clinically detailed simulation model for the United States heart allocation system.

Simulation modeling has been widely used by researchers to evaluate different organ allocation policies. The first simulation model was developed in 1995 for the liver allocation system by Pritsker et al. (1995), and later evolved into what is known today as SRTR Liver and Kidney-Pancreas Simulated Allocation Models 
(LSAM and KPSAM, respectively). The TSAM simulation model was later added to this package. For the kidney and liver allocation systems several researchers have developed simulation models to evaluate alternative allocation policies. For example, for the U.S. liver allocation system, discrete-event simulation models have been developed (Kreke et al., 2002; Shechter et al., 2005; Alagoz et al., 2005). For the U.S. kidney allocation system, Monte Carlo and discrete event simulation models have been developed (Zenios et al., 1999; Davis et al., 2013a,b).

Additionally, a recent study developed a kidney allocation system by replicating KPSAM to evaluate different allocation policies (Sandıkçı et al., 2019).

The only simulation model developed for the heart allocation system in the United States was proposed by Hasankhani and Khademi (2017). They developed a discrete event simulation to investigate three alternative allocation policies. Their work overcame some of the limitations of TSAM; however, it does not support heterogeneous zonal structure.

For developing our simulation model, we share the basic structure of TSAM. Our model makes it possible to evaluate heart allocation policies, prior to any potential implementation, and, therefore, to provide a decision support tool to the U.S. heart transplant policy makers and researchers.

\section{B Heart Allocation System}

The U.S. heart allocation system uses transplant candidates, donors characteristics, and geographic classification to allocate hearts to transplant candidates. In particular, transplant candidates registered on the waiting list are prioritized based on their medical condition, distance from available organ, compatibility with the organ donor, and waiting time.

The OPTN policy prioritizes candidates for a herat transplant based on their medical status and their distance from the donor hospital. Tables 1 and 2 in Chapter II show the sequence for the heart allocation system. The policy excludes 
all the candidates who are not compatible with the organ donor in terms of blood type, weight, height, age, and possibly gender, if there is any requirement on gender for a specific candidate. After screening out incompatible candidates, if there are multiple candidates with the same characteristics eligible for receiving the organ, then the organ would first be offered to the candidate with the longest waiting time. The candidate can accept or decline the offer based on the organ characteristics and the survival benefit. The organ will be offered to the candidates on the priority list until someone accepts the organ; otherwise the organ will be discarded.

If a candidate accepts the offer, then the organ will be sent to the transplant center, where the transplant candidate is registered, for transplantation. If, after the transplant, the organ fails and the transplant recipient survives, he/she will be added to the transplant list again.

\section{Simulation Framework}

We designed a discrete event simulation model for heart allocation system to assess alternative heart allocation policies, which are not feasible with TSAM. From here, we refer to our simulation model as HASim (Heart Allocation Simulation Model). We have independently replicated most of the concepts in TSAM (TSAM, 2017). Similar to TSAM we extensively used historical data on heart transplantation in the United States.

Figure 36 shows the simulation framework of the U.S. heart allocation system, which is triggered by three main events: (i) patient arrival, (ii) patient status update, and (iii) organ arrival. The specific components of the simulation model are described next.

\section{Patient Arrival}

Patients enter the system in three ways: (i) patients on the initial waiting list, (ii) patients who register on the transplant list during the study period, and 


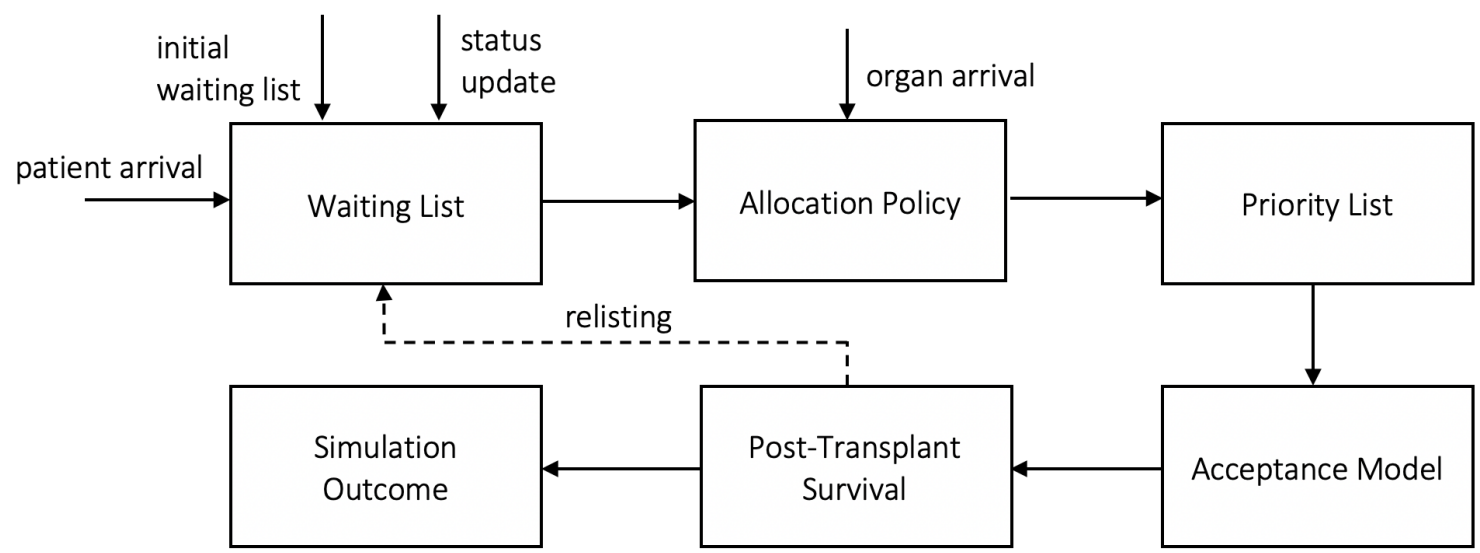

Figure 36. Simulation framework for the heart allocation system in the United States

(iii) patients who after receiving a heart transplant faces graft failure, which is estimated by the survival module in Section 4 .

The initial waiting list is the list of patients who are already waiting for a transplant when simulation starts. New patients are added to the initial waiting list based on their arrival time in the system. The initial waiting list and the list of patients who register on the transplant waiting list during the study period, are generated as input data. For each record of patients, we assigned a total of 38 characteristics, including age, weight, height, patient status, gender, registered transplant center, OPO, arrival time, among others. The arrival time for patients relisted in the waiting list is calculated based on graft's survival time, which is estimated by the post-transplant survival module.

\section{Patient Status Update}

When patients are registered on the waiting list, they are assigned a status related to their medical condition. However, this medical status for patients may change while they are waiting for a transplant.

We imported historical information on patients status changes, generated from the OPTN data, into the model. During the simulation this module updates 
the patients' status if they are still on the waiting list. Some patients who are waiting for a transplant might be removed from the waiting list due to worsening their condition or other reasons, or they might die while they are waiting for transplant. In these cases, this module removes the patient from transplant the waiting list.

\section{Organ Arrival}

Upon each organ arrival, a total of 33 organ characteristics is considered for the organ. Then, the module looks for a match for the organ. To do so, it creates a priority list of patients based on the sequence specified in the allocation policy, and, if the list contains no patients, the module moves to the next allocation sequence and creates a new priority list. The module offers the recovered organ to the patient on the top of the priority list. Then, it calculate the probability of acceptance for the patient, to estimate if the patient will accept the offer or not. The module continues offering the recovered organs until one of these two cases happens: (i) a patient accepts the organ, or (ii) the number of offers exceeds a user-specified upper limit. In, the following, we explain the priority list and acceptance model as sub-modules for the organ arrival.

\subsection{Priority List}

Creating a priority list of patients for a specific organ depends on the sequence specified in the allocation policy. The module generates such a list, based on the allocation zone, blood type compatibility, and patient medical status requirement. When this list is created, all the patients who are not compatible with the organ based on characteristics such as height, weight, and age are removed from this list and then the module sort the patients based on their waiting times. 


\subsection{Acceptance Model}

Whenever an offer has been made, this module calculates the probability of the patient accepting the offered organ. TSAM computes such a probability as a function of several factors related to the organ and donor characteristics, potential organ recipient's characteristics, and factors specific to the compatibility to donor-recipient pair. If we define $x_{i}$ as the scalar variable related to the patient's characteristics, and $y_{j}$ as the scalar variable related to the organ's characteristics, then acceptance probability is $\sum_{i} \sum_{j} \beta_{i j} x_{i} y_{j}$, where $\beta_{i j}$ is the coefficient for related to the specific donor-recipient pair. In our model, we used the same coefficients as TSAM. For each organ offer, this equation is calculated, and the resulting value is transformed using an inverse logit transformation:

$$
\frac{\exp \left(\sum_{i} \sum_{j} \beta_{i j} x_{i} y_{j}\right)}{1+\exp \left(\sum_{i} \sum_{j} \beta_{i j} x_{i} y_{j}\right)} .
$$

That value is then compared to a random variate from a Uniform $(0,1)$ distribution. If it is greater than the random number, then the organ is accepted; otherwise, it is rejected.

\section{Post Transplant Graft Survival}

When a patient receives a graft, this module estimates the time, $T$, remaining before death. We used the same parametric models used by TSAM to determine the post-transplant events and to estimate the corresponding time until failure (TSAM, 2017). TSAM uses a cox regression model to estimate the survival time. $T$ calculated as:

$$
\text { Prob }[\text { survival time }>T]=S(t)^{\sum_{i} \sum_{j} \alpha_{i j} x_{i} y_{j}}
$$

Where $S(t)$ is a step function that can be defined by the user, $S(t)$ denotes the probability of survival after $t$ days from the transplant. Similar to the acceptance model, $x_{i}$ and $y_{j}$ are scalar variables related to patient's and organ's characteristics, 
and $\alpha_{i j}$ is the donor-recipient coefficient. The values we used for the coefficients for the $\alpha_{i j}$ are the same of those used in TSAM.

Then, at each transplantation event, the model determines the remaining survival time by sampling a value $u$ from a Uniform $(0,1)$ distribution and inverting the complementary cumulative probability distribution for the survival time for this patient:

$$
\begin{aligned}
& \text { Prob }[\text { survival time }>T]=u \\
& T=S^{-1}\left(\frac{\exp (\ln (u))}{\exp \left(\sum_{i} \sum_{j} \alpha_{i j} x_{i} y_{j}\right)}\right)
\end{aligned}
$$

To perform this inversion, the model first computes the value of the transformed probability $v$ :

$$
\left.v=\exp \left(\sum_{i} \sum_{j} \alpha_{i j} x_{i} y_{j}\right)\right)
$$

which results in $0<v<1$. The model then uses the step function to determine the corresponding survival time.

\section{Simulation Outcomes}

According to the survival time estimation, the simulation outcome are:

- Transplant recipient and graft survival: the graft estimated failure time is after the end of simulation period. Thus, transplant recipient with his/her graft survives throughout the rest of the simulation period.

- Death: graft fails and the recipient dies before the end of the simulation.

- Relisting: graft fails but the recipient survives and he/she is added to the transplant waiting list again. 


\section{Model Validation}

We used the simulation output files to validate the model on multiple performance metrics against TSAM outcomes to ensure that the model adequately represents the heart allocation system. To do so, we ran the simulation model for 10 replications with the current allocation policy that uses homogeneous zonal structure. The homogeneous zonal structure is the same across the donor hospitals and consist of local area and five concentric circle with radii of 500, 1000, 1500, 2500, and outside 2500 miles. We ran HASim using the three patient status definition (i.e., status 1A, 1B, and 2). For model validation we used TSAM input data from July $1^{\text {st }}, 2009$ to June $30^{\text {th }}$, 2011. This data contained 2,554 transplant candidates on the initial waiting list, 5,864 new candidates to be added to the transplant list, and 3,770 heart organs to be recovered during the simulation. More information about TSAM input data is provided in Appendix B.

Table 21 shows the simulation outcomes from TSAM, and from HASim and the relative error between them, computed as:

$$
\text { Relative Error }=\frac{H A S i m-T S A M}{H A S i m}
$$

We performed a t-student test to evaluate whether the HASim outcomes is significantly different from TSAM. The null hypothesis in this test is that HASim and TSAM are different, and a p-value $>0.05$ rejects the null hypothesis. The p-value resulted from the t-student test showed that the overall outcome from HASim is not significantly different from TSAM.

Table 22 shows the number of transplants on different groups of patients resulted from TSAM, HASim, and the relative difference. Similarly, Table 23 shows the number of death among the transplant candidates for TSAM and HASim. The results for model validation clearly illustrate that HASim outputs closely match TSAM (except for status 2 recipients). 
TABLE 21

Model validation on different simulation outcomes.

\begin{tabular}{lllll}
\hline & TSAM & HASim & Relative Error & P-value \\
\hline Transplant & 3,734 & 3,711 & $-0.61 \%$ & 0.773 \\
Death & 861 & 846 & $-1.89 \%$ & 0.472 \\
Removed & 834 & 854 & $2.34 \%$ & 0.386 \\
Waiting & 2,992 & 3,007 & $0.49 \%$ & 0.740 \\
\hline
\end{tabular}

\section{E Heterogeneous vs Homogeneous Zonal Structure}

We used HASim to compare the optimized heterogeneous zonal structure, designed in Chapter III, with the current homogeneous zonal structure. We ran HASim for 10 replications. The main focus of our analysis was to compare these two systems in terms of geographic disparity in access to heart transplants. For this purpose, we presented the results of our evaluation in term of transplant rate, waiting time and pre-transplant mortality rate and average travel distance. It should be noted that we ran the simulation for these two systems based on the same allocation policy and the same patients status definition. The only difference between these two systems was the radius for the zones around donor hospitals. Additionally, we just considered the first three zones around donor hospitals. The allocation policy inside the first three zones around donor hospitals is provided in Appendix B.

For Our analysis we used OPTN data set from 2011 to 2014. This data contains 11,560 transplant candidates who registered on the heart transplant waiting list during the study period, and 8,130 recovered heart organs. We did not consider data in hospitals and transplant centers in Puerto Rico, Hawaii, and Alaska. Additionally, we did not consider pediatric candidates (i.e., age $<18$ ), multi-organ transplant candidates (i.e., heart-lung), and candidates that were on the waiting list prior to January 2011. More information about the input data is provided in Appendix B. 
TABLE 22

Model validation on number of heart transplants based on patients status, age, blood type, and year.

\begin{tabular}{llllll}
\hline & & TSAM & HASim & Relative Error & P-Value \\
\hline Status & $1 \mathrm{~A}$ & 2,133 & 2,055 & $-3.79 \%$ & 0.249 \\
& $1 \mathrm{~B}$ & 1,414 & 1,407 & $-0.49 \%$ & 0.898 \\
& 2 & 187 & 249 & $24.80 \%$ & 0.004 \\
\hline Age & $18-34$ & 370 & 365 & $-1.36 \%$ & 0.866 \\
& $35-48$ & 701 & 703 & $0.28 \%$ & 0.958 \\
& $49-63$ & 1,868 & 1,819 & $-2.69 \%$ & 0.398 \\
& $64-74$ & 795 & 824 & $3.50 \%$ & 0.436 \\
\hline Blood Type & $\mathrm{A}$ & 1,515 & 1,537 & $1.43 \%$ & 0.693 \\
& $\mathrm{~B}$ & 550 & 560 & $1.78 \%$ & 0.750 \\
& $\mathrm{AB}$ & 216 & 221 & $2.26 \%$ & 0.812 \\
& $\mathrm{O}$ & 1,455 & 1,393 & $-4.45 \%$ & 0.253 \\
\hline Year & 2009 & 902 & 894 & $-0.89 \%$ & 0.835 \\
& 2010 & 1,889 & 1,880 & $-0.47 \%$ & 0.865 \\
& 2011 & 903 & 937 & $3.62 \%$ & 0.740 \\
\hline
\end{tabular}

Table 24 shows the summary statistics of the transplant rate, the waiting time, the pre-transplant mortality rate, and the travel distance for homogeneous and heterogeneous zonal structure. For each metric the table reports the average, standard deviation (STD), interquartile range (IQR; i.e., between $25^{\text {th }}$ and $75^{\text {th }}$ percentiles), and range across transplant centers. Figure 37 shows the distribution of transplant rate, waiting time, and pre-transplant mortality rate, and travel distance across transplant centers.

The average transplant rate for homogeneous and heterogeneous zonal structures was the same. Similarly, the STD, IQR, and range of transplant rate did not change when adopting one of the two zonal structures.

For waiting time, with heterogeneous zonal structure the average waiting time increased for 8 days, while the standard deviation in waiting time across transplant centers decreased from 24.34 to 19.75. Additionally, the maximum waiting time across transplant center for homogeneous zonal structure was 145 days 


\section{TABLE 23}

Model validation on number of deaths based on patients status, age, blood type, and year.

\begin{tabular}{llllll}
\hline & & TSAM & HASim & Relative Error & P-Value \\
\hline Status & 1A & 54 & 59 & $8.47 \%$ & 0.674 \\
& 1B & 100 & 106 & $5.66 \%$ & 0.684 \\
& 2 & 64 & 62 & $-3.22 \%$ & 0.856 \\
& inactive & 315 & 328 & $3.96 \%$ & 0.597 \\
\hline Age & $18-34$ & 90 & 86 & $-4.65 \%$ & 0.732 \\
& $35-48$ & 146 & 136 & $-7.35 \%$ & 0.588 \\
& $49-63$ & 414 & 409 & $-1.22 \%$ & 0.862 \\
& $64-74$ & 212 & 201 & $-5.47 \%$ & 0.497 \\
\hline Blood Type & $\mathrm{A}$ & 287 & 280 & $-2.50 \%$ & 0.774 \\
& $\mathrm{~B}$ & 81 & 74 & $-9.45 \%$ & 0.507 \\
& $\mathrm{AB}$ & 22 & 18 & $-22.22 \%$ & 0.497 \\
& $\mathrm{O}$ & 472 & 460 & $-2.60 \%$ & 0.697 \\
\hline Year & 2009 & 201 & 194 & $-3.60 \%$ & 0.736 \\
& 2010 & 418 & 413 & $-1.19 \%$ & 0.829 \\
& 2011 & 243 & 225 & $-8.00 \%$ & 0.404 \\
\hline
\end{tabular}

and for heterogeneous zonal structure was 95 days.

The average pre-transplant mortality rate for both homogeneous and heterogeneous zonal structures was the same. However, with heterogeneous zonal structure the standard deviation in mortality rate decreased from 0.15 to 0.02 , and the maximum mortality rate across transplant centers decreased from 0.33 to 0.20 .

With heterogeneous zonal structure the average travel distance decreased from 304.32 to 289.89 miles. Additionally, heterogeneous zonal structure decreased the standard deviation in travel distance from 156.05 to 96.75 miles, and the maximum travel distance across transplant centers decreased from 1,485 to 663 miles. 


\section{TABLE 24}

Comparison between homogeneous and heterogeneous zonal structures in terms of transplant rate, waition time, mortality rate, and travel distance.

\begin{tabular}{ll|ll}
\hline & & Homogeneous & Heterogeneous \\
\hline Transplant Rate & Average & 0.65 & 0.64 \\
& STD & 0.14 & 0.13 \\
& IQR & 0.15 & 0.15 \\
& Range & $0.28-0.90$ & $0.30-0.91$ \\
\hline Waiting Time & Average & 59.00 & 67.00 \\
& STD & 24.34 & 19.75 \\
& IQR & 28.94 & 26.87 \\
& Range & $1-145$ & $1-95$ \\
\hline Pre-transplant Mortality Rate & Average & 0.07 & 0.06 \\
& STD & 0.15 & 0.02 \\
& IQR & 0.04 & 0.03 \\
& Range & $0-0.33$ & $0-0.20$ \\
\hline Travel Distance (mile) & Average & 304.32 & 289.89 \\
& STD & 156.05 & 96.75 \\
& IQR & 142.02 & 153.67 \\
& Range & $0-1,485$ & $0-663$ \\
\hline
\end{tabular}

\section{F Conclusion}

The heart allocation system is complex in nature and implementing new changes requires careful consideration. Simulation modeling has been considered as an efficient way for assessing and evaluating possible changes in the allocation systems before being implemented.

In this chapter we developed a simulation model for the heart allocation system in the United States as an alternative to TSAM. We replicated most of TSAM features and adding more flexibility to evaluate alternative allocation policies. We validated HASim with TSAM. We performed the validation on overall outcomes and on the outcome for different groups of patients. The results from our validation indicated that HASim clearly represented the U.S. heart allocation system. 


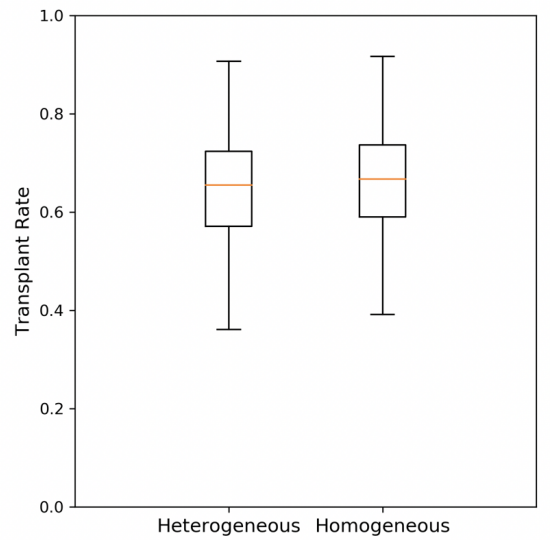

(a)

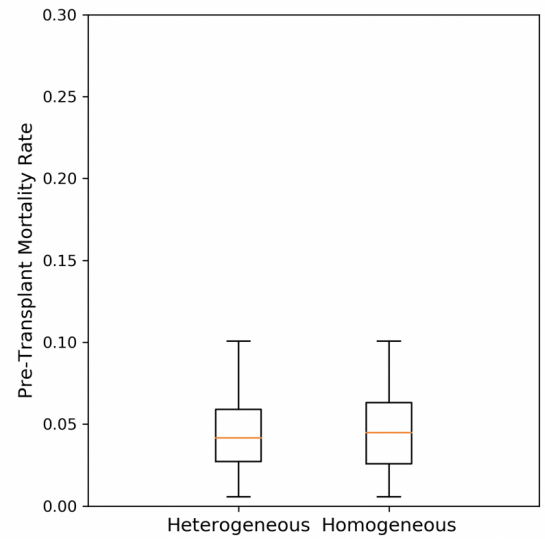

(c)

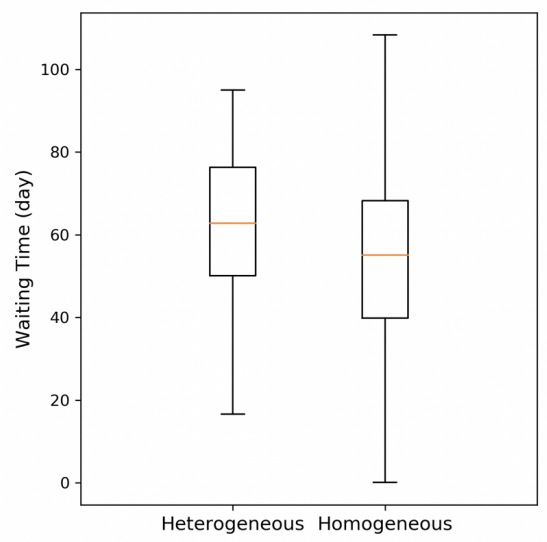

(b)

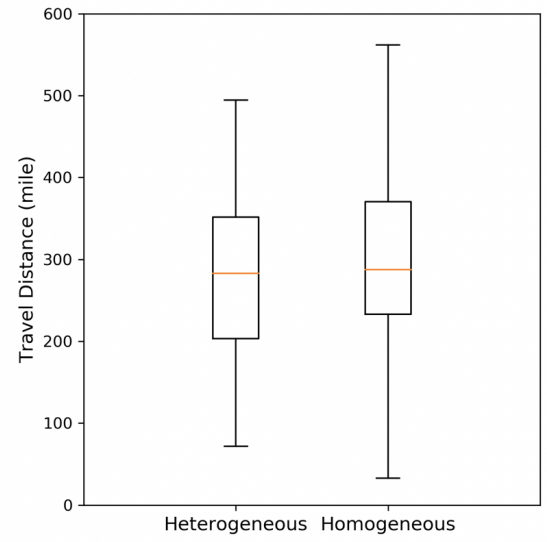

(d)

Figure 37. Comparison between homogeneous and heterogeneous zonal structures in terms of (a) transplant rate, (b) waiting time, (c) pre-transplant mortality rate, and (d) travel distance.

We used HASim to compare a heterogeneous zonal structure versus a homogeneous zonal structure one, in terms of disparity in access to a transplant. We measured access using different metrics, including, transplant rate, waiting time to a transplant, pre-transplant mortality rate and average travel distance. Our analysis indicated that with a heterogeneous zonal structure disparity in transplant rate across transplant centers did not change, however, disparity in terms of waiting time, pre-transplant mortality rate and average travel distance decreased. It should be noted that the average waiting time increased slightly (8 days) with a 
heterogeneous zonal structure. On the other hand, the average travel distance and the maximum travel distance decreased from 156.05 to 96.75 miles and from 1,485 to 663 miles, respectively. Even though with heterogeneous zonal structure we allocated organs maximum in 1,200 miles from donor hospitals (as oppose to homogeneous zonal structure with three zones that allocates organs within 1,500 miles), the total transplant rate was $86 \%$, which is the same as homogeneous zonal structure.

Our analysis showed that a customized zonal structure, based on supply and demand distribution reduces disparity. We outline that, in our analysis, given the available data, we considered a three status grouping of the patients (i.e., Status 1A, $1 \mathrm{~B}$ and 2), however, an analysis with the current grouping of patients in six status should be performed. Unfortunately, currently the OPTN data does not provide information on the new patient status definition, however HASim provides the opportunity for this analysis.

There are some limitations related to HASim. First, we used the historical data for patients and organ arrivals, and for updating patient status. Using historical data is more accurate for estimating the events in the simulation model. However, it is not sufficient for predicting future changes. Second, patients who underwent transplants in real life have no status updates in their patient records after the transplant date. The historical date on status update is incomplete. Third, with HASim organs are discarded after a fixed number of declined offers, regardless of organ and donor characteristics.

In this study, we developed a simulation model for the U.S. heart allocation system and validated its performance. We used this model to evaluate heterogeneous zonal structure, and the simulation result indicated that the optimized heterogeneous zonal structure reduces geographic disparity in travel distance, waiting time and pre-transplant mortality rate. 


\section{CHAPTER V}

\section{OPTIMAL INTEGRATION OF DESENSITIZATION PROTOCOLS INTO KIDNEY PAIRED DONATION (KPD) PROGRAMS}

\section{A Introduction}

Blood type (ABO) incompatibility and antibody to donor human leukocyte antigen (HLA) remain the most significant barriers in transplantation. Literature reports (Ferrari et al., 2014) that both access to and outcomes of kidney transplants are worse among those patients with high degrees of ABO and HLA incompatibilities (ABOi and HLAi, respectively). The degree of histocompatibility barriers is measured using an immunological lab test known as Panel Reactive Antibody (PRA). The PRA score ranges between $0 \%$ and 100\%, and the transplant candidates with a PRA score of $80 \%$ or higher are defined as "highly sensitized" patients. Sensitization is commonly acquired as a result of pregnancy, transfusion, prior transplant, and viral/bacterial infections. Highly sensitized patients were known to wait significantly longer on a deceased donor kidney waitlist (Haririan et al., 2009), at least until the 2014 kidney allocation policy change that prioritized them on the waitlist, and their risk of post-transplant graft failure is reported to be substantially higher than other patients (Zachary and Leffell, 2014; Mattsson et al., 2008; Marfo et al., 2011). Similarly, patients with blood type O tend to experience more difficulty getting a donor and thus tend to have a longer wait time (Wolfe et al., 2000; Crew and Ratner, 2010; Kannabhiran et al., 2012). In the United States, there is about a $35 \%$ chance that any two people are ABOi, and about a $30 \%$ chance that a transplant candidate is sensitized (Montgomery et al., 2011). For 
these patients, available options to improve both access to and outcomes of transplants are desensitization and kidney paired donor (KPD) exchange, the system that allows incompatible donor-patient pairs to exchange their living donors with other incompatible pairs within the program.

Desensitization of transplant candidates was first introduced about two decades ago as a promising treatment to overcome histocompatibility barriers (Wallis et al., 2011; Gentry et al., 2011). The protocols of desensitization are performed prior to transplantation to reduce/eliminate incompatibilities and donor specific antibody through a series of immunosuppression regimens. Despite the recent advancement in the desensitization protocol, challenges remain among highly sensitized patients. Literature suggests (Zachary and Leffell, 2014) that the desensitization protocols alone may not be able to reduce the antibody level enough to make a transplant possible for these patients. In these cases, undergoing desensitization as well as identifying a more compatible donor via KPD exchange is recognized as a novel promising approach (Montgomery et al., 2005; Montgomery, 2010; Montgomery et al., 2011). KPD exchange was first introduced in 2000 in the United States as a way to overcome ABOi and HLAi between patients and their living donors (Montgomery et al., 2011). The patients undergoing the combination of desensitization and KPD can be desensitized more efficiently to meet the compatibility requirements (Montgomery et al., 2012). Early evidence suggests that the combination of the two procedures leads to an increase of the transplant rates (Montgomery et al., 2005; Montgomery, 2010; Montgomery et al., 2011).

Motivated by this current status, the goal of this paper is to develop a simulation-optimization approach to optimally incorporating desensitization into a KPD exchange and evaluating the benefit of such an integration. In this study, we specifically focus on ABOi desensitization. This is because the structure of ABOi is less complex with ABO antibody titers being the only element, while HLAi desensitization needs to take compatibilities of several antigen classes as well as 
donor specific antibodies into account. Moreover, a recent large-scale study reports that the transplant outcomes of ABOi and ABO compatible transplants (in terms of both patient mortality and graft failure) are comparable (Opelz et al., 2015; Montgomery et al., 2012). In terms of desensitization for HLAi, the transplant community seems to have more reservation due to the relatively poor long-term transplant outcomes (Pankhurst et al., 2017; Lefaucheur et al., 2010). Thus, focusing on ABOi in this exploratory study allows us to develop an optimization model without compromising the realism of desensitization impacts and the outcomes.

Prior operations research literature on KPD investigates the optimal strategy to match donors to patients within a given set of incompatible pairs (Dai and Tayur, 2018; Anderson et al., 2015; Constantino et al., 2013). The corresponding optimization problem is known in the literature as the Kidney Exchange Problem (KEP), which consists in determining the maximum number of transplants which can be performed among a pool of incompatible patient-donor pairs. Several mathematical formulations have been proposed (Anderson et al., 2015; Constantino et al., 2013; Mak-Hau, 2017) to model KEP. These existing studies exclusively look for possible matches without considering the possibility of combining KPD and desensitization, often resulting in less than $15 \%$ of highly sensitized patients finding a compatible pair in a match run (Montgomery et al., 2011). In this study, we adapt the existing models to overcome this limitation by including the possibility that some of the patients in the pool could undergo a desensitization protocol to improve their compatibility with the matched donor via KPD. We embed the model into a simulation framework and perform an extensive experimental analysis to evaluate the impact of optimally integrating a desensitization protocol in a KPD program. The outcome measures of our analysis are:

- Increase in the transplant rate,

- Increase in the number of transplants among highly sensitized patients, 
- Increase in the number of transplants among blood type O patients,

- Decrease in wait time for a transplant.

The reminder of the chapter is organized as follows. In Section B, we formally describe the Kidney Exchange Problem (KEP) and provide details on how existing mathematical models can be adapted to integrate desensitization. Section $\mathrm{C}$ contains the description of our experimental setting. The results are presented in Section D. Discussion of the results, limitation of the approach, and further extensions are the objectives of Section E.

\section{B Optimization Model}

A possible run of a KPD can be modeled using a graph representation (hereafter referred to as compatibility graph) such as the one shown in Figure 38. Each node $i$ in the graph represents an incompatible patient-donor pair $\left\{p_{i}, d_{i}\right\}$. There are 7 incompatible pairs represented in the graph. An arc $(i, j)$ from node $i$ to node $j$ means that donor $d_{i}$ is compatible with patient $p_{j}$. For example, arc $(1,2)$ in the graph represents compatibility between donor $d_{1}$ and patient $p_{2}$.

A possible kidney exchange is shown in Figure 38b, where dark lines represent the resulting matches of the run. In the example, the solution of the run has two cycles of length three (the length of a cycle is the number of arcs/nodes it contains). In particular, one of the cycles contains arcs $(4,5),(5,3)$ and $(3,4)$, which correspond to the matches $d_{4}-p_{5}, d_{5}-p_{3}$, and $d_{3}-p_{4}$ (i.e., donor $d_{4}$ is matched with patient $p_{5}$, donor $d_{5}$ is matched with patient $p_{3}$, and donor $d_{3}$ is matched with patient $\left.p_{4}\right)$. The other cycle contains arcs $(1,2),(2,6)$, and $(6,1)$; that is donor $d_{1}$ is matched with patient $p_{2}$, donor $d_{2}$ is matched with patient $p_{6}$, and donor $d_{6}$ is matched with patient $p_{1}$. Patient $p_{7}$ and donor $d_{7}$ (in node 7 ) are not matched and would have to wait for the next run. Hence, in this example, a total of six transplants will be performed (the total number of dark lines). 


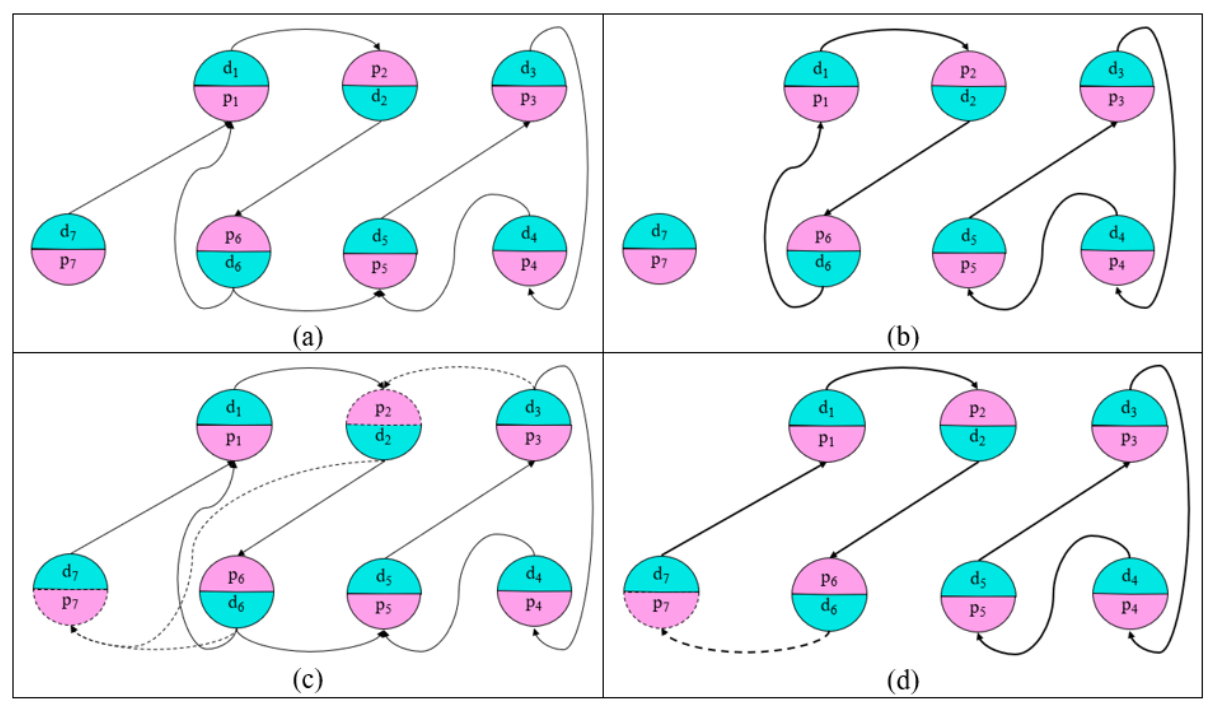

Figure 38. Example of a KPD match run representation: (a) compatibility graph; (b) example of a resulting matching of a KPD run without integration with a desensitization protocol; (c) extended compatibility graph; (d) example of a resulting matching of a KPD run integrated with a desensitization protocol

In order to account for the possibility that a patient in the pool could undergo a desensitization process to increase its compatibility in the pool, we modified the compatibility graph by adding new arcs to the graph (hereby referred to as treatment arcs). We refer to the resulting graph as the extended compatibility graph. The set of treatment arcs represents the new compatibilities among patients and donors which can be possible for those patients who undergo desensitization. Let us assume, in our example, the possibility that patients $p_{2}$ and $p_{7}$ can undergo a desensitization therapy. The resulting extended compatibility graph is show in Figure 38c where three treatment arcs (represented by the dotted lines) were added to the graph: $(3,2),(2,7)$ and $(6,7)$. Specifically, if the patient denoted by $p_{7}$ is desensitized, then $\mathrm{s} /$ he would become compatible with donor $d_{2}$ and donor $d_{6}$. Similarly, if patient $p_{2}$ is desensitized, s/he would become compatible with donor $d_{3}$. A kidney exchange in this extended compatible graph, not only determines the possible matches among compatible pairs, but also determines, among the set of the patients in the pool, who should be treated with a desensitization protocol. For 
example, Figure 38d represents a possible kidney exchange (represented by the dark lines) in the extended compatibility graph. This solution requires that patient $p_{7}$ undergoes desensitization and that a kidney exchange be performed through a cycle of length four (namely, $d_{1}-p_{2}, d_{2}-p_{6}, d_{6}-p_{7}$, and $d_{7}-p_{1}$ ), and another cycle of length three (namely, $d_{3}-p_{4}, d_{4}-p_{5}$, and $d_{5}-p_{3}$ ). Hence, by allowing the integration, a total of seven transplants will be performed.

There are several proposed mathematical formulations in the literature to solve KEP on a given compatibility graph, the literature usually includes a limit on the maximum length of a cycle allowed in the solution because of logistical reasons such as the simultaneous availability of operating rooms (Ferrari et al., 2009; Johnson et al., 2008; Roth et al., 2007; De Klerk et al., 2010; Lee et al., 2009; Montgomery et al., 2008). In our experiments, we implemented and solved the cycle formulation Constantino et al. (2013), which is presented next after introducing some needed notation. Let $G(V, A)$ be the compatibility graph associated with a given pool of donor-patients pairs, where $V$ is the set of nodes representing donor-patient pairs, and $A$ is the set of arcs. Let $A^{+}$be the set of treatment arcs, which represents new compatibilities in the pool due to the $\mathrm{ABO}$ desensitization treatment. This set of arcs is defined by the KPD program in accordance with specific requirements and goals of the program as well as the consent of the patients. For example, a transplant program can decide to propose desensitization to the patients whose PRA is above a given threshold value, or to blood type $\mathrm{O}$ patients. The extended compatibility graph $G^{+}\left(V, A \cup A^{+}\right)$is obtained by adding the set of treatment $\operatorname{arcs} A^{+}$to the initial set of $\operatorname{arcs} A$. Let $C$ be the set of all cycles in $G^{+}$, and $C_{k} \subseteq C$ be the subset of cycles with length less than or equal to a given threshold $k$. We denote $C_{k}(v)$ the cycles from $C_{k}$ containing an arc incident to $v$. Let $w_{i j}, \forall(i, j) \in A \cup A^{+}$be a weight associated with each arc $(i, j)$ in the graph. These weights are usually used to define the importance (priority) of a transplant. We define the weight $w_{c}$ of a given cycle $c \in C$ as the sum of the weights of the arcs 
that the cycle contains, i.e., $w_{c}=\sum_{(i, j) \in C} w_{i j}$. Finally, let us consider the set of binary variables $z_{c}, \forall c \in C_{k}$, associated with each cycle $C$ in $C_{k}$, whose value is equal to 1 if cycle $C$ is selected to be in the solution and zero otherwise. KEP on $G^{+}$can be formulated as follows:

$$
\operatorname{Max}_{z_{c}} \sum_{c \in C_{k}} w_{c} z_{c}
$$

subject to

$$
\begin{aligned}
& \sum_{c \in C_{k}(v)} z_{c} \leq 1, \forall v \in V \\
& z_{c} \in\{0,1\} . \forall c \in C_{k}
\end{aligned}
$$

Model 15 - 17 selects the maximum weight node disjoint cycle packing of the extended compatibility graph, where cycles can contain at most $k$ nodes. Specifically, the objective function 15 maximizes the total number of (weighted) transplants in the solution. If the weights are equal to one, i.e., $w_{i j}=1, \forall(i, j) \in A \cup A^{+}$, then the objective function maximizes the total number of transplants. Constraint 17 ensure that a node belongs to no more that one of the selected cycles.

KEP can be easily solved to optimality when only cycles of length two (i.e., $k=2$ ) are allowed, however, it was shown to be NP-hard even when only cycles of length two and three are used (i.e., $k=3$ ) (Abraham et al., 2007), meaning that it is unlikely there will be an algorithm that always finds the optimal solution quickly in every instance of the problem. However, integer programming (IP) formulations, as the one provided here, have been widely used by a variety of authors to solve special cases of the KEP (Constantino et al., 2013). In our experimentation, $k$ was set to a maximum value equal to six, which is realistic from a logistical point of view (apart from very special cases (Sack, 60), the length of the cycles is usually limited to either 3 or 4 (Constantino et al., 2013)). In addition, the size of the pool in our experiments is composed of at most 124 incompatible pairs (i.e., the number of 
nodes in the underlying graph is at most equal to 124), which is also deemed realistic (Gentry et al., 2007). Given this setting, we were able to always solve the model to optimality. We implemented Model 15 - 17 in Python 2.7 and solved it by means of the solver Gurobi 7.0.2.

\section{Experimental Analysis}

To evaluate the impact of optimally integrating an ABO desensitization protocol in a KPD program we performed a series of experiments with different scenarios. The details of the used data, scenarios characteristics, simulation strategy, and output measures are provided in the following sections.

\section{Data}

We used data (provided by the authors of (Anderson et al., 2015)) from two major KPD programs in the United States: the National Kidney Registry (NKR), and the Alliance for Paired Donation (APD). The data contained the information of 826 incompatible donor-patients pairs covering a period of 2.5 years. Of the 826 patients, 403 were highly sensitized (patients with PRA greater than or equal to 80). For each pair, the data contained patient and donor blood type, patients' PRA, and pair's historical arrival time. The resulting compatibility graph contained 115,605 arcs.

\section{Scenarios}

We considered 16 different scenarios by varying: (i) the maximum length $k$ of a cycle (i.e. $k \in\{3,4,5,6\}$ ) admitted in a KPD run, and (ii) the number $\delta$ of generated treatment arcs. Specifically, we generated the extended compatibility graph by adding treatment arcs resulting from ABOi desensitization. We used KPSAM (ver. 4.1.5, the Kidney-Pancreas Simulated Allocation Model) to obtain a proxy measure for additional compatibilities made possible by removing ABOi. 
KPSAM is a simulation software developed by the US Scientific Registry of Transplant Recipients (SRTR) (SAM, 2017), it is available for researchers upon request and is widely used for the impact evaluation of different kidney allocation protocols at the national level (Israni et al., 2014a). KPSAM is preloaded with the existing kidney allocation rules and the UNOS national transplant registry data. For the current study, we used the allocation rules embedded in KPSAM as the default using data covering years from 2007 to 2008. In order to estimate the additional compatibility arcs that are made possible via desensitization for ABOi, we modified the definition of ABO compatibility as shown in Figure 39.

\begin{tabular}{|c|c|c|c|c|c|c|c|c|c|}
\hline \multicolumn{5}{|c|}{ Original / Baseline ABO Compatibility* } & \multicolumn{5}{|c|}{ Modified ABO Compatibility* } \\
\hline $\begin{array}{l}\text { Blood } \\
\text { Type }\end{array}$ & 0 & A & B & $A B$ & $\begin{array}{l}\text { Blood } \\
\text { Type }\end{array}$ & 0 & A & B & $A B$ \\
\hline 0 & I & $C / X$ & $\mathrm{C} / \mathrm{X}$ & $c / X$ & 0 & $\mathrm{I}$ & C & C & $\mathrm{C}$ \\
\hline A & $\mathrm{x}$ & I & $\mathrm{X}$ & $\mathrm{C}$ & A & C & I & C & $\mathrm{C}$ \\
\hline B & $x$ & $\mathrm{x}$ & $\mathrm{I}$ & $\mathrm{C} / \mathrm{X}$ & B & $\mathrm{C}$ & C & I & $\mathrm{C}$ \\
\hline$A B$ & $x$ & $x$ & $x$ & 1 & $A B$ & C & C & C & 1 \\
\hline
\end{tabular}

Figure 39. Modification in the ABO compatibility definition in KPSAM

The simulation was ran one time for two scenarios, one with the aforementioned modification, and another keeping the default compatibility definition. The simulation results indicated that the average increase in compatibilities as the result of ABOi desensitization was around $11 \%$ of the initial set of arcs. Given that these numbers serve as an approximate and that the actual increase in the compatibility arcs depends on other donor and patient factors (such as $\mathrm{ABO}$ antibody titers and donor specific antibody), we tested three different integrated-scenarios by randomly generating a number of treatment arcs equal to $\delta=2 \%, \delta=6 \%$ and $\delta=11 \%$ of the initial set of arcs, with the $\delta=11 \%$ case being the most optimistic scenario. A scenario with $\delta=0 \%$ corresponds to the baseline-scenario. 


\section{Simulation Strategy}

We adapted the simulation strategy used by Anderson et al. (2015) for simulating the dynamic environment of a kidney exchange pool. An overview of the adopted strategy for a given scenario is described in Figure 40. The simulation performs the following six steps:

1. A set of treatment arcs is randomly generated according to the value of parameter $\delta$.

2. Incompatible pairs are randomly assigned an arrival time by shuffling their historical arrival time.

3. Eight new participants are added, according to their arrival time, to form the current exchange pool.

4. The mathematical $15-17)$ is solved for the current pool of incompatible pairs.

5. All the matched nodes are removed from the pool.

6. Steps 3-5 are repeated until no new more participants can be added to the pool.

For a given scenario, every time the above six steps are performed, we have a trial. In each trial, when new incompatible pairs enter the system and the pool is updated (Step 3), we say a new snapshot is created. Since the data contains 826 pairs and each time eight pairs enter the system, each trial contains 104 snapshots (i.e., the nearest largest integer of the ratio $\frac{826}{6}$ ). 


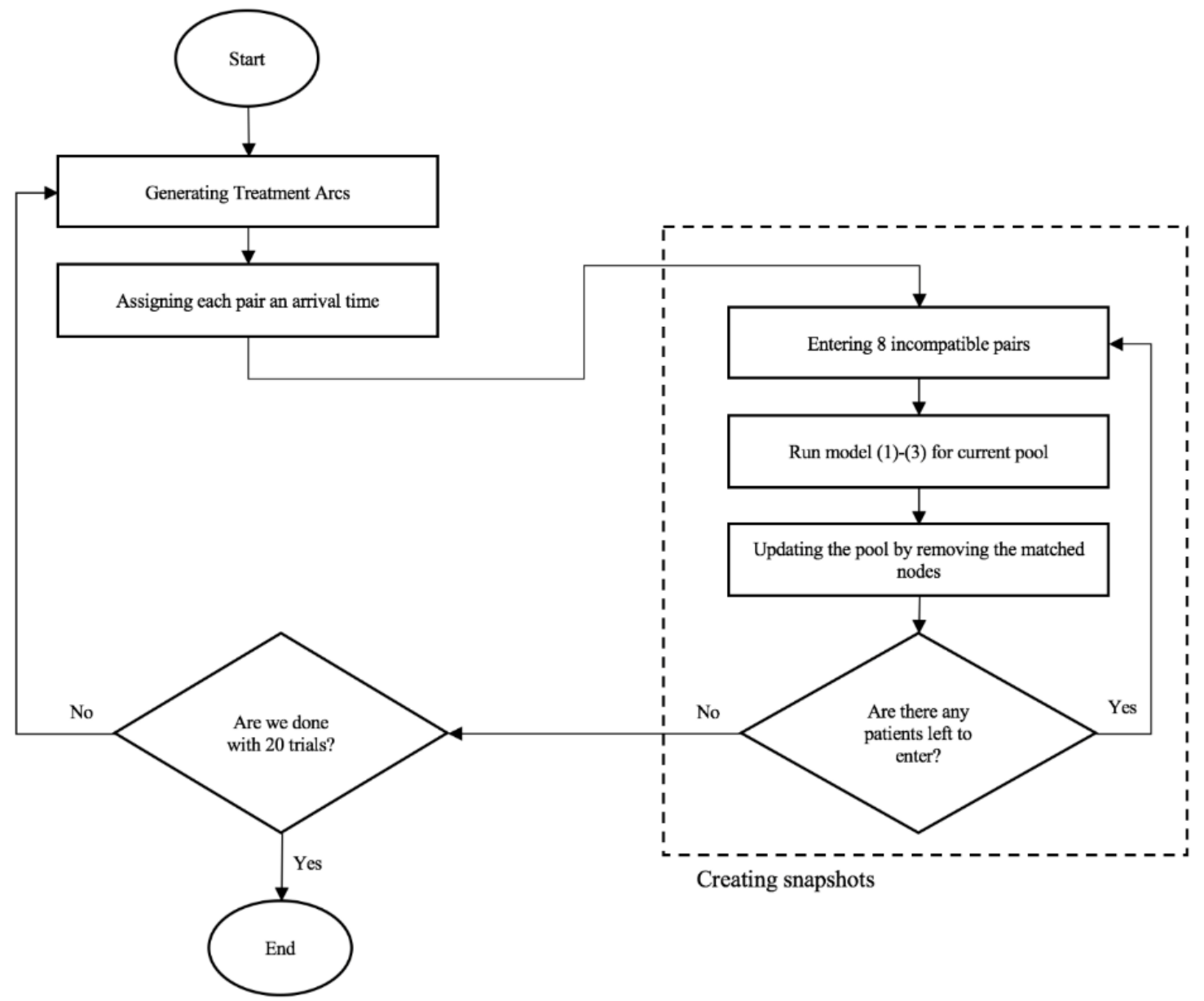

Figure 40. Adopted Simulation Strategy

We ran the described procedure to simulate both a KPD program with ABOi desensitization (integrated-scenario), and a KPD program without desensitization (baseline-scenario). The difference is in the underling graph on which the mathematical model, solved in Step 4 of the above described procedure, is applied. Specifically, the model is solved on the extended compatibility graph for the integrated-scenario runs, and on the initial compatibility graph for the baseline-scenario runs. 


\section{Output Measures}

In the analysis of the results we considered three groups of patients based on their PRA: (i) patients with $P R A \geq 80$, who are highly sensitized patients (80-Level patients), (ii) patients with $50<P R A<80$ (50-Level patients), and (iii) patients with $P R A<50$ (0-Level patients). The following statistics are computed to evaluate the impact of the integration:

1. Transplant rate for all patients. Transplant rate for each trial is computed as the ratio between the total number of transplants performed in the trial and the total number of incompatible pairs.

2. Transplant rate for 80-Level (50-Level, 0-Level) patients. The transplant rate of 80-Level patients (50-Level, 0-Level), in a given trial, is computed as the ratio between the total number of matched 80-Level (50-Level, 0-Level) patients and the total number of 80-Level (50-Level, 0-Level) patients in the system.

3. Transplant rate for patients with blood type $O$ (blood type $A, B$, and $A B$ ). The transplant rate for blood type $\mathrm{O}(\mathrm{A}, \mathrm{B}, \mathrm{AB})$ patients is computed as the ratio between the total number of matched blood type $\mathrm{O}(\mathrm{A}, \mathrm{B}, \mathrm{AB})$ patients and the total number of blood Type $\mathrm{O}(\mathrm{A}, \mathrm{B}, \mathrm{AB})$ patients in the system.

4. Number of snapshots during which highly sensitized patients remain in the system. For each trial, we record the total number of snapshots each highly sensitized patient remains in the system before being matched.

\section{Results}

The results of our analysis are shown in Tables 25 - 29 and Figures 41 - 43 . Table 25 shows, for each scenario, the average transplant rates (computed among the 20 trials of the scenario) of: (i) all patients; (ii) patients with different PRA 
level; and (iii) patients with different blood types. The baseline-model corresponds to the rows in the table labeled with $\delta=0 \%$. As expected, all the transplant rates increased with respect to the baseline-scenario both when cycle length increased, and when a greater number of treatment arcs was added to the initial compatibility graph. In particular, if we consider the transplant rate for all patients under the integrated-scenario $\delta=11 \%$, the rate of incompatible pairs which can be matched ranged between 0.93 (when $k=3$ ) and 0.96 (when $k=6$ ). If we look at the transplant rates for the difficult to match patients (that is 80-Level and blood type O patients), we can observe when $k=3$ and $\delta=2 \%$ (which is the most conservative scenario), the transplant rate of matched 80-Level patients was 0.65 (0.36 for the baseline-scenario), and the transplant rate of matched blood type $\mathrm{O}$ patients was 0.72 ( 0.36 for the baseline-scenario). These rates increase up to 0.94 , and 0.96 , respectively, when $k=6$ and $\delta=11 \%$. From these results we can observe that the impact of longer cycles on the number of transplants is more evident when the number of treatment arcs is low (i.e., the advantage of allowing longer chain when with $\delta=2 \%$ is greater than when $\delta=11 \%$ ). This shows that the increase in compatibility among pairs due to the addition of the treatment arcs is much more impactful than the use of longer cycles, in other words, desensitizing a greater number of patients improves the transplant rate dramatically and it eliminates the need of longer cycles.

The impact of the integration is evident from Figure 41, which shows the boxplots, over the 20 trials, of the percentage increase of the transplant rates, with respect to the baseline-scenario, for all the integrated-scenarios. Boxplots are shown considering different patient groups (i.e., all patients, patients with different PRA level, and patients with different blood types). The percentage increase is computed as the ratio $\left(\frac{X_{I}-X_{B}}{X_{B}}\right) * 100$, where $X_{I}$ is the value of the statistic computed under an integrated-scenario, and $X_{B}$ is the value of the statistic computed under the baseline-scenario. The median of the percentage increase in the transplant rate for 
all patients ranges between $66 \%$ (when $k=3$ ) and $68 \%$ (when $k=6$ ) when $\delta=2 \%$, and between $99 \%$ (when $k=6$ ) and $111 \%$ (when $k=3$ ) when $\delta=11 \%$.

From Figure 41, it is evident that the integration of desensitization in a KPD program affects highly sensitized patients (i.e., 80-level patients) and blood type O patients the most. Indeed, the median percentage increase in the transplant rate for 80-Level patients ranged between $76 \%$ (when $k=6$ and $\delta=2 \%$ ) and $148 \%$ (when $k=3$ and $\delta=11 \%$ ), for 50-Level patients the transplant rate ranged between $27 \%$ (when $k=3$ and $\delta=2 \%$ ) and $47 \%$ (when $k=3$ and $\delta=11 \%$ ), for 0-Level patients it ranged between $69 \%$ (when $k=3$ and $\delta=2 \%$ ) and $106 \%$ (when $k=3$ and $\delta=11 \%$ ). If we look at the transplant rate increment for different blood types, for blood type A patients the transplant rate ranged between $29 \%$ (when $k=3$ and $\delta=2 \%$ ) and $58 \%$ (when $k=3$ and $\delta=11 \%$ ), for blood type B patients it ranged between $38 \%$ (when $k=3$ and $\delta=2 \%$ ) and $75 \%$ (when $k=3$ and $\delta=11 \%$ ), for blood type $\mathrm{AB}$ patients it ranged between $38 \%$ (when $k=3$ and $\delta=2 \%$ ) and $71 \%$ (when $k=3$ and $\delta=11 \%$ ), and finally, for blood type $\mathrm{O}$ patients it ranged between $103 \%$ (when $k=3$ and $\delta=2 \%$ ) and $167 \%$ (when $k=3$ and $\delta=11 \%$ ).

The driver for such a significant increase is due to the change in the structure of the underlying graph when treatment arcs are added to the pool. Even with $2 \%$ added treatment arcs, the number of cycles in the graph increases exponentially, hence the probability of finding compatible donors drastically increases. Table 26 shows this characteristic. The table displays the average number of cycles in the underlying graph for all the scenarios. The average was computed among 20 randomly generated graphs from the historical dataset containing 50, 100 and 150 incompatible pairs. 
TABLE 25

Average of the transplant rates for, computed among the 20 trials of each scenario

\begin{tabular}{|c|c|c|c|c|c|}
\hline Transplant Rate & $\delta$ & $k=3$ & $k=4$ & $k=5$ & $k=6$ \\
\hline All & $0 \%$ & 0.44 & 0.46 & 0.47 & 0.48 \\
\hline \multirow[t]{3}{*}{ Patients } & $2 \%$ & 0.73 & 0.78 & 0.80 & 0.81 \\
\hline & $6 \%$ & 0.87 & 0.91 & 0.92 & 0.93 \\
\hline & $11 \%$ & 0.93 & 0.95 & 0.96 & 0.96 \\
\hline 80-Level & $0 \%$ & 0.36 & 0.40 & 0.42 & 0.43 \\
\hline \multirow[t]{3}{*}{ Patients } & $2 \%$ & 0.65 & 0.72 & 0.75 & 0.76 \\
\hline & $6 \%$ & 0.82 & 0.87 & 0.89 & 0.90 \\
\hline & $11 \%$ & 0.90 & 0.93 & 0.94 & 0.94 \\
\hline 50-Level & $0 \%$ & 0.65 & 0.67 & 0.67 & 0.68 \\
\hline \multirow[t]{3}{*}{ Patients } & $2 \%$ & 0.83 & 0.87 & 0.89 & 0.89 \\
\hline & $6 \%$ & 0.92 & 0.94 & 0.95 & 0.96 \\
\hline & $11 \%$ & 0.96 & 0.97 & 0.97 & 0.97 \\
\hline 0-Level & $0 \%$ & 0.47 & 0.48 & 0.48 & 0.48 \\
\hline \multirow[t]{3}{*}{ Patients } & $2 \%$ & 0.80 & 0.84 & 0.85 & 0.86 \\
\hline & $6 \%$ & 0.92 & 0.94 & 0.95 & 0.95 \\
\hline & $11 \%$ & 0.96 & 0.97 & 0.97 & 0.98 \\
\hline Blood Type A & $0 \%$ & 0.58 & 0.60 & 0.62 & 0.62 \\
\hline \multirow[t]{3}{*}{ Patients } & $2 \%$ & 0.76 & 0.80 & 0.82 & 0.83 \\
\hline & $6 \%$ & 0.86 & 0.90 & 0.92 & 0.92 \\
\hline & $11 \%$ & 0.92 & 0.94 & 0.95 & 0.95 \\
\hline Blood Type B & $0 \%$ & 0.52 & 0.56 & 0.58 & 0.59 \\
\hline \multirow[t]{3}{*}{ Patients } & $2 \%$ & 0.73 & 0.80 & 0.82 & 0.82 \\
\hline & $6 \%$ & 0.85 & 0.89 & 0.91 & 0.91 \\
\hline & $11 \%$ & 0.92 & 0.94 & 0.95 & 0.95 \\
\hline
\end{tabular}



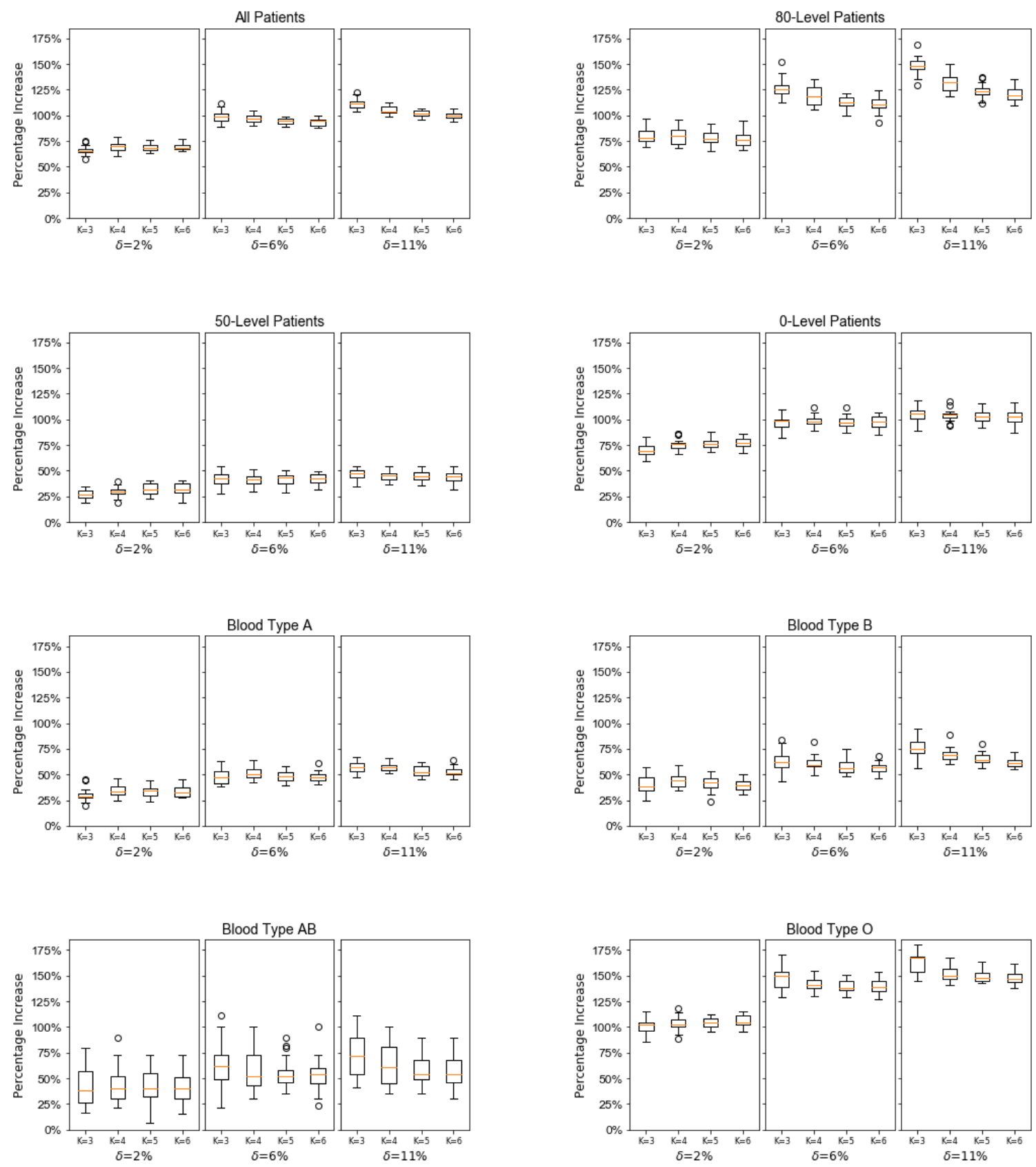

Figure 41. Percentage increase in transplant rates for all patients, for patients with different PRA level, and for patients with different blood types with respect to different value of $k$, and when $\delta=2 \%, \delta=6 \%$ and $\delta=11 \%$.

Integration of desensitization in a KPD program will benefit the patients 


\section{TABLE 26}

Average number of cycles in the underlying graph for all the scenarios. The average is computed among random graphs, containing 50, 100 and 150 incompatible pairs

\begin{tabular}{llllll}
\hline $\begin{array}{l}\text { Number of } \\
\text { Nodes }\end{array}$ & $\delta$ & $k=3$ & $k=4$ & $k=5$ & $k=6$ \\
\hline 50 & $0 \%$ & 60 & 225 & 790 & 2,681 \\
& $2 \%$ & 67 & 264 & 990 & 3,666 \\
& $6 \%$ & 86 & 362 & 1,481 & 5,990 \\
& $11 \%$ & 122 & 585 & 2,789 & 13,413 \\
\hline 100 & $0 \%$ & 402 & 2,887 & 21,332 & 161,544 \\
& $2 \%$ & 461 & 3,492 & 27,217 & 216,892 \\
& $6 \%$ & 620 & 5,256 & 46,552 & 423,275 \\
& $11 \%$ & 852 & 8,080 & 80,058 & 813,303 \\
\hline 150 & $0 \%$ & 1224 & 13,061 & 143,596 & $1,600,321$ \\
& $2 \%$ & 1436 & 16,292 & 190,999 & $2,282,196$ \\
& $6 \%$ & 1934 & 24,466 & 324,362 & $4,409,516$ \\
& $11 \%$ & 2692 & 38,383 & 572,600 & $8,779,067$ \\
\hline
\end{tabular}

most in need, not only because their chance of getting a transplant increases, but also because they will stay in the system less. As explained in the previous section, in each trial, a sequence of snapshots is created. When a snapshot is created, some of the patients are matched and leave the system while others remain in the system and are included in the next snapshot which is created by adding eight new incompatible pairs. For each trial, we recorded the number of snapshots that a highly sensitized patient remains in the pool before s/he is matched with a compatible donor. Then, for each scenario, we computed the average among the 20 trials, and converted that average to a proportion. The cumulative distribution of those proportions is shown in Figure 42. Specifically, each sub-figure shows the cumulative percentage of 80-Level patients who are matched in less than a given number of snapshots. Each diagram shows the distribution of the integrated-scenarios $(\delta=2 \%, \delta=6 \%$ and $\delta=11 \%)$ and of the baseline-scenario $(\delta=0 \%)$ for different values of $k$. From the figure we can observe that, for any 
value of $k$, as $\delta$ increases, the number of snapshots required by 80 -Level patients to be matched decreases. For example, when $k=3$, in the baseline-scenario, $12 \%$ of the highly sensitized patients were matched in the very first snapshot in which they participated (number of snapshots in the $\mathrm{x}$-axis is equal to 1 ), while this proportion was equal to $23 \%$ for the integrated-scenario with $\delta=2 \%$, and $29 \%$ and $32 \%$ for the integrated-scenario when $\delta=6 \%$ and $\delta=11 \%$, respectively. In the baseline-scenario with $k=3$, only $34 \%$ of the highly sensitized patients were matched in less than 50 snapshots, while in the integrated-scenarios this proportion was equal to $61 \%, 80 \%$ , and $89 \%$ when $\delta=2 \%, \delta=6 \%$, and $\delta=11 \%$ respectively.
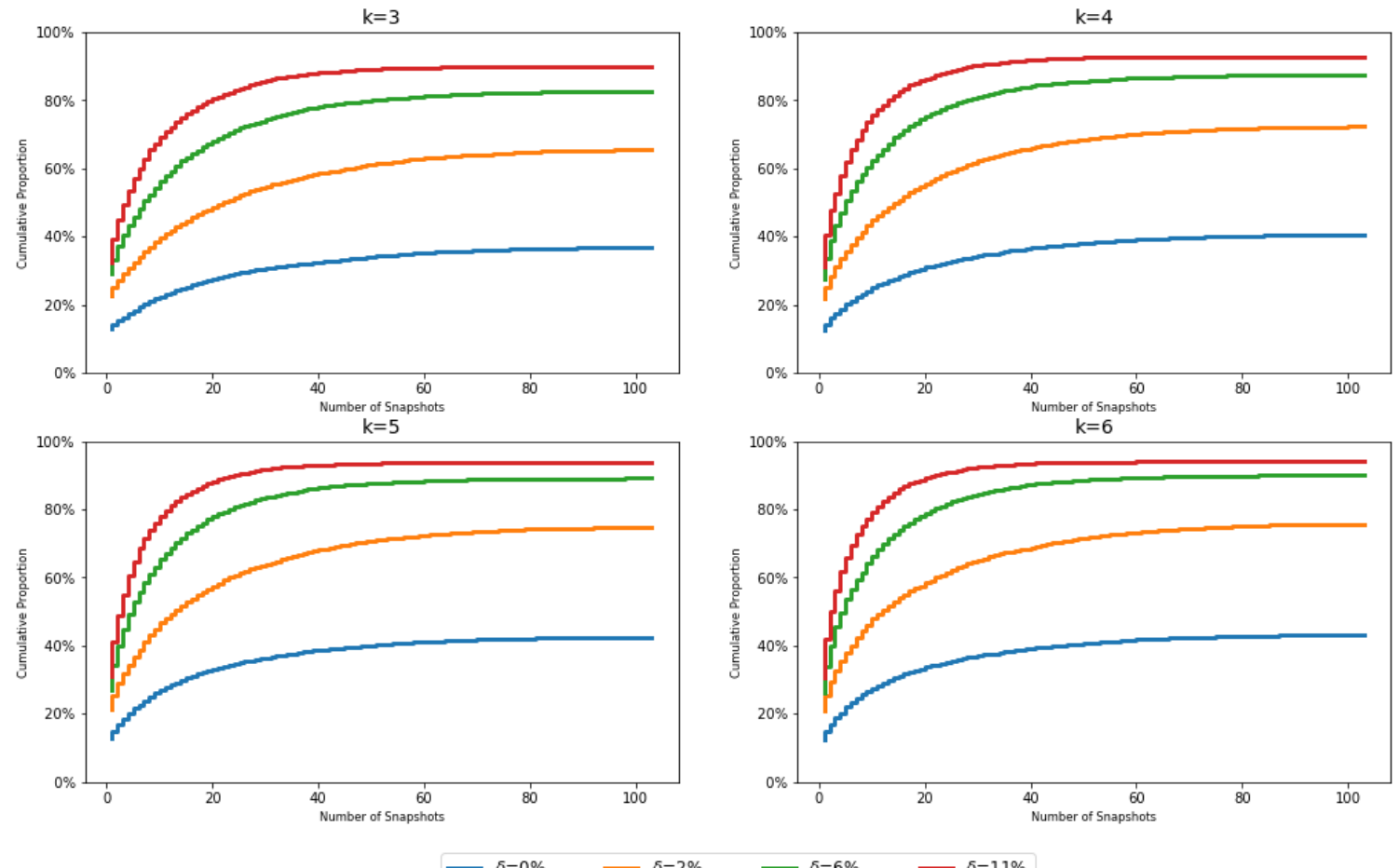

$-\delta=0 \% \quad-\delta=2 \% \quad-\delta=6 \% \quad-\delta=11 \%$

Figure 42. Comparison between the baseline-scenario and the integrated-scenarios of the cumulative percentage of 80 -Level patients who are matched in less than a given number of snapshots

Tables 27-tab:desensitizedrate-bldtype and Figure 43 give an overall picture of the penetration of the desensitization in the KPD program. Table 27 shows the average percentage, computed from the 20 trials of each scenario, of matched 
patients who underwent desensitization. Table 28 shows the average percentage of treated patients with respect to different levels of PRA (i.e., 80-level, 50-level, and 0-level patients). Table 29 shows analogous average percentages computed by patient's blood type. Note that, the percentage of desensitized patients ranged between 0.32 (Table 27, $\delta=2 \%, k=3$ ) and 0.53 (Table $27, \delta=11 \%, k=6$. When looking at the categories of patients (Table 28 and Table 29), we can see that the highest percentage of desensitized patients are the 80-Level and the blood type $\mathrm{O}$ patients. Figure 6 reports the average percentage of cycles containing at least one patient who undergoes desensitization. For example, when $\delta=2 \%$ and $k=3,58 \%$ of the cycles of length 2 contained at least one desensitized patient, $76 \%$ of the cycles of length 3 contained at least one desensitized patient. Figure 43 demonstrates that, for every integrated scenario, longer cycles are more likely to contain a patient who underwent desensitization, and this likelihood increases with $\delta$.

\section{TABLE 27}

Percentage of matched patients who undergo desensitization for different scenarios

\begin{tabular}{cccccc}
\hline Treated Rate & $\delta$ & $k=3$ & $k=4$ & $k=5$ & $k=6$ \\
\hline All Patients & $2 \%$ & 0.32 & 0.35 & 0.35 & 0.36 \\
& $6 \%$ & 0.44 & 0.46 & 0.47 & 0.47 \\
& $11 \%$ & 0.51 & 0.52 & 0.53 & 0.53 \\
\hline
\end{tabular}

\section{E Conclusion}

The present study demonstrated that combining ABO blood group incompatible desensitization therapy with KPD allocation strategy will offer immediate opportunities to perform more transplants. Our results indicate that, even with a limited number of patients undergoing a desensitization treatment and without allowing too long cycles, on average, one can expect $66 \%$ increase in the transplant rate, $79 \%$ increase in the number of highly sensitized patients 
TABLE 28

Percentage of matched 80-level, 50-level, and 0-level patients who undergo desensitization for different scenarios

\begin{tabular}{cccccc}
\hline Treated Rate & $\delta$ & $k=3$ & $k=4$ & $k=5$ & $k=6$ \\
\hline 80-Level & $2 \%$ & 0.68 & 0.65 & 0.63 & 0.62 \\
Patients & $6 \%$ & 0.69 & 0.66 & 0.66 & 0.65 \\
& $11 \%$ & 0.68 & 0.66 & 0.66 & 0.65 \\
\hline 50-Level & $2 \%$ & 0.08 & 0.08 & 0.08 & 0.08 \\
Patients & $6 \%$ & 0.09 & 0.08 & 0.08 & 0.08 \\
& $11 \%$ & 0.09 & 0.09 & 0.09 & 0.09 \\
\hline 0-Level & $2 \%$ & 0.24 & 0.27 & 0.29 & 0.30 \\
Patients & $6 \%$ & 0.23 & 0.25 & 0.26 & 0.27 \\
& $11 \%$ & 0.23 & 0.24 & 0.25 & 0.26 \\
\hline
\end{tabular}

transplanted, and $101 \%$ increase in the number of blood type $O$ patients transplanted. While further model development and analyses are necessary to provide practical insights into the KPD exchange programs, our results reveal that desensitization administered as part of a KPD exchange program is a promising approach to improve access to and to reduce wait time for a living donor kidney transplant.

The present study is the first optimization attempt to examine the role of desensitization in a KPD exchange program even though the model is experimental and preliminary. The major limitation lies in the fact that our experiment does not account for several realistic aspects. Our model does not account for: (i) the initial $\mathrm{ABO}$ antibody titer level as well as the amount and cost of required desensitization given the titer; (ii) the level of initial HLA mismatches and the presence of donor-specific antibodies (DSAs) as well as the associated desensitization cost; and (iii) possible failure of either desensitization or a transplant.

Incorporating $\mathrm{ABO}$ titer and HLA distributions as well as DSA information in the model is not an easy task partially because such information is not readily 
TABLE 29

Percentage of matched patients who undergo desensitization for different scenarios

\begin{tabular}{cccccc}
\hline Treated Rate & $\delta$ & $k=3$ & $k=4$ & $k=5$ & $k=6$ \\
\hline Blood Type A & $2 \%$ & 0.2 & 0.19 & 0.19 & 0.19 \\
Patients & $6 \%$ & 0.21 & 0.21 & 0.21 & 0.21 \\
& $11 \%$ & 0.23 & 0.22 & 0.23 & 0.23 \\
\hline Blood Type B & $2 \%$ & 0.11 & 0.11 & 0.11 & 0.1 \\
Patients & $6 \%$ & 0.12 & 0.12 & 0.12 & 0.12 \\
& $11 \%$ & 0.13 & 0.13 & 0.13 & 0.13 \\
\hline Blood Type AB & $2 \%$ & 0.01 & 0.01 & 0.01 & 0.01 \\
Patients & $6 \%$ & 0.01 & 0.01 & 0.01 & 0.01 \\
& $11 \%$ & 0.02 & 0.01 & 0.02 & 0.02 \\
\hline Blood Type O & $2 \%$ & 0.69 & 0.69 & 0.69 & 0.70 \\
Patients & $6 \%$ & 0.66 & 0.66 & 0.66 & 0.66 \\
& $11 \%$ & 0.63 & 0.63 & 0.63 & 0.63 \\
\hline
\end{tabular}

available from the UNOS national transplant registry database. From the limited data in our center (i.e., The George Washington University Hospital), the percentages of those patients who are relatively hard to desensitize (i.e., titer $\geq 64$ ) are about $8 \%$ for the blood type $\mathrm{A}$ (anti-B) and $\mathrm{B}$ (anti-A1 or A2) recipients and about $60 \%$ for the blood type $\mathrm{O}$ (anti-A1, A2 or B) recipients. For these patients, desensitization is performed with plasmapheresis over a period of time as far as the patients has a living-donor and the original titer level is not too high (i.e., >512). In terms of the desensitization targeting HLA antibodies, the patients with higher PRA are generally harder to desensitize, and low PRA patients with high levels of cross-match reactivity to common HLA types are also difficult to desensitize (Gloor et al., 2010).

The cost of transplantation would be inevitably high for these patients. Currently, in our center a typical bortezomib-based induction therapy increases the cost of transplantation by $\$ 10,000$ to $\$ 13,000$. A round of plasmapheresis (if the 

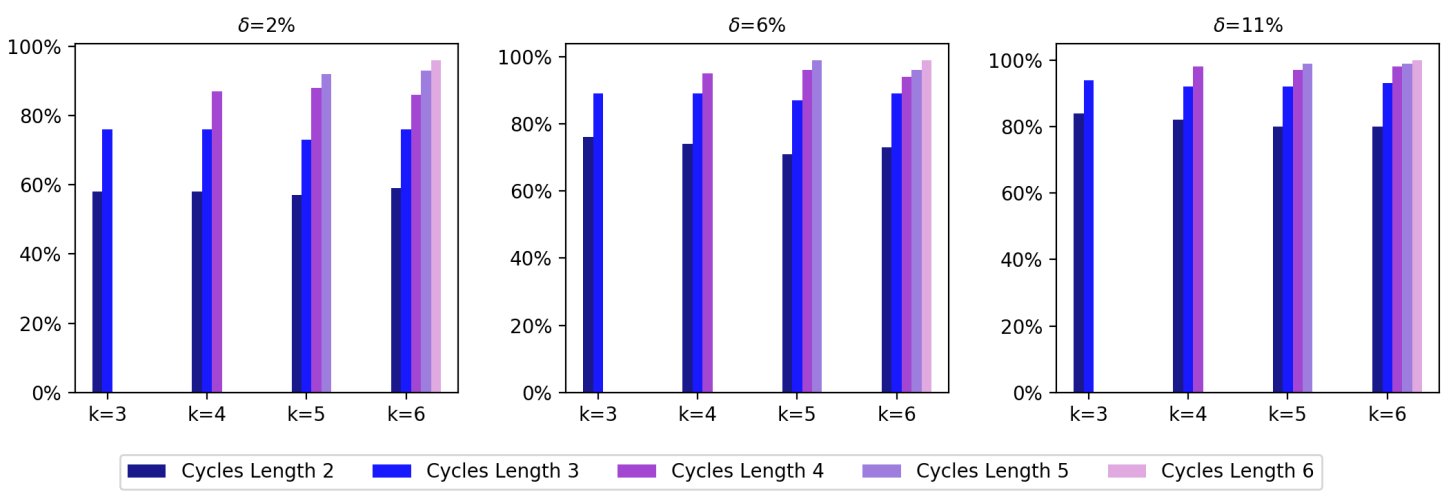

Figure 43. Average percentage of cycles that contains at least one treatment arc for $k \in\{3,4,5,6\}$ and $\delta=2 \%, \delta=6 \%, \delta=11 \%$.

titer is above 64 ) adds additional $\$ 1,000$ to $\$ 2,000$ to the cost. Estimating the total desensitization cost for the patients with various profiles would require further data collection and analyses. Similarly, the HLA mismatch level and the DSA prevalence for the patients with a given blood type and ABO titer would require further data collection and analyses. Incorporating these heterogeneities in the levels of antibodies and desensitization costs in the model will be immediate steps to extend our model. The extensions would require more complex calculations to be performed.

Including transplant outcomes with a different desensitization level in the model is another important step to make the model more realistic. Some literature suggests that transplant outcomes among desensitized patients, especially the highly sensitized patients, are poorer than those patients who received transplants without desensitization (Haririan et al., 2009; Montgomery et al., 2011; Ferrari et al., 2009). However, in terms of the ABO antibody desensitization, evidence suggests that both short- and long-term patient mortality and graft failure rates do not differ between $\mathrm{ABO}$ compatible transplants and $\mathrm{ABO}$ incompatible transplants with desensitization (Opelz et al., 2015; Montgomery et al., 2012). In contrast, evidence on the inferior outcomes of HLA incompatible transplants seem to be well 
established (Pankhurst et al., 2017; Lefaucheur et al., 2010). The outcomes of ABO + HLA incompatible transplants are reported to be similar to the outcomes of HLA incompatible transplants, suggesting that there are no synergistic effects between anti- blood group and HLA antibodies (Couzi et al., 2015).

The current study focused on the impact of combining KPD and ABO antibody desensitization on the efficiency of kidney transplants. Given that the desensitization is possible for most KPD patients in the absence of DSA and high level HLA mismatches, we opine that the estimated increase in the transplant rate presented here is not too far from the reality. Whether such a combined therapy is cost effective for all patients regardless of the titer level is another question. To explore such a question, however, further data collection and analysis will be required to estimate wait time for a living donor with a better match or for a deceased donor transplant as well as the cost of dialysis and clinical implications of the prolonged time on dialysis.

It should also be noted that the expected increase in transplants reported here is a long-run outcome, and the time it takes to reach the long-run state depends on how quickly transplant centers are willing to explore the combined procedure, especially the ABOi transplants. In December 2014, the new national kidney allocation system (KAS) was introduced to support transplants between A2/A2B deceased donors and B patients (Martins et al., 2018). The number of A2/A2B to B transplants has been growing for the last 4 years. However, the growth rate remains around $1 \%$ per annum as the speed of accommodating this new policy differs by transplant center.

Finally, many KPD programs specify a hierarchy of allocation criteria (Lee et al., 2009; Manlove and O'Malley, 2012; Kim et al., 2007; Glorie et al., 2014) which considers several factors in addition to the total number of transplants. Such factors include prioritization of highly sensitized patients and of patients with low match probability, length of the cycles and of the chains, patients' wait time, age of 
patients and donors, travel distance between matched pairs, quality adjusted life years, etc. Our model can also be extended to account for multiple objectives to account for these factors.

Despite the limitations and extensions warranted to fully understand the desensitization effects, we consider that our finding provides meaningful implications and direction for the future KPD research, especially in light of ever-exacerbating access to both living and deceased donor renal transplant and the recent nationwide attention to the KPD exchange system. 


\section{CHAPTER VI}

\section{ALLOCATING KIDNEYS IN OPTIMIZED HETEROGENEOUS CIRCLES}

\section{A Introduction}

Geographic residence is the most important determinant of access to kidney transplant in the United States (Zhou et al., 2018; Formica, 2018). There is more variation in transplant access attributable to donor service areas (DSA) than any

other candidate characteristic, such as calculated panel reactive antibody (cPRA), blood type, kidney disease etiology, and socioeconomic status(Stewart et al., 2018). In July 2018, the Secretary of Health and Human Resources advised the Organ Procurement and Transplant Network (OPTN) to eliminate DSAs and regions and develop allocation schemes consistent with the Final Rule (DHHS), which states that organ allocation should not be based on waitlisted candidate's residence or transplant center listing.

The OPTN Kidney-Pancreas Workgroup initially put forth a "hybrid" allocation framework using a 500 nautical mile (nm) fixed-distance circle around each donor hospital with continuous score priority for candidates nearer to donor hospital (OKT). In public comments, the proposal was criticized for not considering the variation in deceased donor supply and organ procurement organization (OPO) donor authorization rates across the country. Commenters also noted that some transplant centers' circles comprised mostly oceans and other countries.

Commenters variously argued for larger circle sizes, given kidneys' ability to tolerate relatively long cold ischemia times with minimal negative patient outcomes (Stewart 
et al., 2016), or for smaller circle sizes to decrease travel burden for rurally located transplant centers. Ultimately, the workgroup settled on a $250 \mathrm{~nm}$ fixed-distance circle solution for all transplant centers.

Heterogeneous circles, that is, circles of different sizes in different places, might better balance supply and demand needs around the country. We used our BOundary Allocation Problem model, that we developed in chapter III to design heterogeneous circles around donor hospitals for kidney allocation and minimize the variability in supply/demand ratios across transplant centers.

\section{B Method}

Data Source We used data from the Scientific Registry of Transplant Recipients (SRTR) that includes data on all donor, wait-listed candidates, and transplant recipients in the U.S., submitted by the members of the Organ Procurement and Transplantation Network (OPTN). The Health Resources and Services Administration (HRSA), U.S. Department of Health and Human Services provides oversight to the activities of the OPTN and SRTR contractors.

Data Population We studied 49,099 transplanted deceased kidney donors and 131,706 waitlist candidates from 236 transplant centers from 2014 to 2017. We excluded discarded kidneys, multi-organ waitlist candidates, inactive candidates, and hospitals in Puerto Rico, Hawaii and Alaska. We used 2017 data for the training set and 2014 to 2016 data for the test sets.

Optimized Heterogeneous Circles Using 2017 data, we used an special case of the BAP model to design heterogeneous circles around donor hospitals to minimize the variation in supply/demand ratios across transplant centers, that is, to minimize the largest difference between any transplant center's supply/demand ratio and the national average supply/demand ratio. The model's constraints ensured that the 
TABLE 30

Numbers of donor hospitals, transplant centers, incident candidates, and deceased donor kidneys transplanted for each year

\begin{tabular}{lcccc}
\hline & 2014 & 2015 & 2016 & 2017 \\
\hline $\begin{array}{l}\text { Number of Transplant } \\
\text { Centers }\end{array}$ & 236 & 238 & 235 & 236 \\
$\begin{array}{l}\text { Incident Active Waitlist } \\
\text { Candidates }\end{array}$ & 32,460 & 33,648 & 33,014 & 34,275 \\
$\begin{array}{l}\text { Number of Donor Hospitals } \\
\text { Deceased Donor Kidneys }\end{array}$ & 1,352 & 1,430 & 1,527 & 1,479 \\
Transplanted & 12,086 & 12,596 & 13,589 & 14,593 \\
\hline
\end{tabular}

circle around each donor hospital included at least one transplant center. Circle size options in the model were 100, 200, 300, 400 and $500 \mathrm{~nm}$. Of those options, the model selected the best circle size for each donor hospital that minimized supply/demand ratio ratios across all transplant centers. The mathematical model for designing optimized heterogeneous circles is presented in Appendix C. After choosing the best circle size for each donor hospital, we compared the results with allocation of kidneys within homogeneous circles of $250 \mathrm{~nm}$ and $500 \mathrm{~nm}$ in size.

Supply/Demand Ratio For each donor hospital, supply was defined as the number of kidneys recovered and transplanted. Demand was the number of active incident kidney waitlist candidates per year, excluding prevalent waitlist candidates (Table 30). The supply/demand ratio for a transplant center was the number of allocated kidneys, divided by number of incident waitlist candidates. We allocated supply proportionate to each transplant center's number of waitlisted candidates. For example, suppose 90 kidneys were recovered in a single donor hospital, and that hospital's allocation circle contained transplant centers A and B. If transplant center A had 70 waitlisted candidates while transplant center B had 30, 70\% of the 90 recovered kidneys would be allocated to transplant center $\mathrm{A}$ and the remainder to transplant center B. 
Average Travel Distance We compared the average distance that a kidney would travel under heterogeneous and homogeneous circle allocation.

\section{Sensitivity Analysis}

- Maximum Circle Size: We tested a range of maximum circle sizes from 200 $\mathrm{nm}$ to $1000 \mathrm{~nm}$, in increments of 100 , to analyze the effect of increasing the allowable circle size on variation of supply/demand ratios and average distance traveled by organs. The minimum circle size was always $100 \mathrm{~nm}$.

- Supply and Demand Changes Over Time: Given that supply and demand naturally vary over time, we investigated whether our optimized heterogeneous circles were robust to changes in supply and demand. Using data from 2017, we designed heterogeneous circles. Then, we calculated the supply/demand ratios using three years of data, 2014 to 2016, that was not used in designing our heterogeneous circles. We compared the variation in supply/demand ratios across transplant centers for heterogeneous circles with homogeneous circles for each of the years.

Statistical Analysis The integer program was solved using the Gurobi 2.7 optimizer with Python 3, yielding an optimal circle size for each donor hospital.

\section{Results}

Optimized Heterogeneous Circles Figure 44 shows the circle size and total supply at each donor hospital. Each point on the map represents a donor hospital and the color shows the circle size for the donor hospital. 14\% of recovered kidneys were distributed within a circle of $100 \mathrm{~nm}, 17 \%$ within a circle of $200 \mathrm{~nm}, 20 \%$ within a circle of $300 \mathrm{~nm}, 17 \%$ within a circle of $400 \mathrm{~nm}$ and $32 \%$ within a circle of $500 \mathrm{~nm}$. 


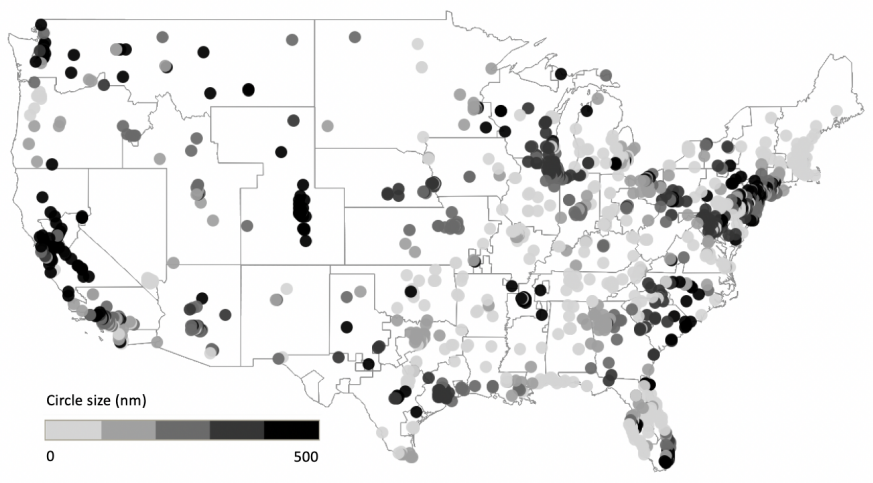

Figure 44. Optimized Heterogeneous Circle Size. Each point represents a donor hospital and the color shows the circle size for the donor hospital.

Supply/Demand Ratio The supply/demand ratios across transplant centers for heterogeneous circles ranged from 0.41 to 0.43 (median 0.42 ) organs per candidate. For $250 \mathrm{~nm}$ homogeneous circles, supply/demand ratios ranged from 0.1 to 0.75 (median 0.42) organs per candidate, and for $500 \mathrm{~nm}$ homogeneous circles, supply/demand ratios ranged from 0.21 to 0.61 (median 0.41 ) organs per candidate (Figure 45).

Average Travel Distance We calculated the average distance traveled by a kidney under homogeneous and heterogeneous circles (Figure 46). Average travel distance is $194 \mathrm{~nm}$ for our heterogeneous circles, $131 \mathrm{~nm}$ for $250 \mathrm{~nm}$ homogeneous circles, and $273 \mathrm{~nm}$ for $500 \mathrm{~nm}$ homogeneous circles. The travel distance across transplant centers ranged from 81 to $288 \mathrm{~nm}$ for our heterogeneous circles, and for $250 \mathrm{~nm}$ homogeneous circles ranged from 38 to $206 \mathrm{~nm}$ and for $500 \mathrm{~nm}$ homogeneous circles ranged from 103 to $374 \mathrm{~nm}$.

\section{Sensitivity Analysis}

Varying Maximum Circle Size We considered optimizing heterogeneous circles using different values for the maximum circle size allowed, and calculated the resulting variation in supply/demand ratios across transplant centers and average 
travel distance. For heterogeneous circles with maximum circle size of $200 \mathrm{~nm}$, supply/demand ratios ranged from 0.15 to 1.00 organs per candidate; for maximum circle size of $500 \mathrm{~nm}$, the ratios ranged from 0.41 to 0.43 organs per candidate; for maximum circle size of $1000 \mathrm{~nm}$, the ratios ranged from 0.41 to 0.44 organs per candidate (Figure 47). Allowing larger circles decreased the variation in supply/demand ratios, but only up to $500 \mathrm{~nm}$. No circles need to be larger than 500 $\mathrm{nm}$ in a heterogeneous circle system to achieve geographic equity in supply/demand ratios.

For heterogeneous circles with maximum circle size of $200 \mathrm{~nm}$ the average travel distance was $72 \mathrm{~nm}$, for maximum circle size of $500 \mathrm{~nm}$ was $194 \mathrm{~nm}$, and for maximum circle size of $1000 \mathrm{~nm}$ was $272 \mathrm{~nm}$ (Figure 48). Allowing larger circles increased the average distance traveled by donor kidneys. The average travel distance for heterogeneous circles is always less than for $500 \mathrm{~nm}$ homogeneous circles. The average travel distance for heterogeneous circles is less than that for 250 $\mathrm{nm}$ homogeneous circles when the maximum circle size is less than or equal to 400 nm.

Supply and Demand Changes Over Time For every year from 2014 to 2017, the heterogeneous circles we designed using 2017 data yielded a much narrower distribution of supply/demand ratios than did homogeneous circles of any size (Figure 49).

\section{E Discussion}

We used an integer-programming model to design heterogeneous circles around donor hospitals that reduced supply/demand ratios variation across transplant centers, as compared to homogeneous circles of size 250 and $500 \mathrm{~nm}$. We found that smarter sharing with heterogeneous circles decreased geographic disparity compared with either size homogeneous circles. Our circles were robust to 
changes in supply and demand over time. Using heterogeneous circles for kidney allocation, no circles need to be larger than $500 \mathrm{~nm}$, and many circles can be smaller than that, while mitigating geographic disparity. More than $50 \%$ of the kidneys were recovered in hospitals with circle size equal to or less than $300 \mathrm{~nm}$. With optimized heterogeneous circles, supply/demand ratios ranged from 0.41 to 0.43 organs per candidate, for $250 \mathrm{~nm}$ homogeneous circles these ratios ranged from 0.10 to 0.75 organs per candidate, and for $500 \mathrm{~nm}$ homogeneous circles these ratios ranged from 0.21 to 0.61 . The average distance a kidney would travel using heterogeneous circles was more than that of $250 \mathrm{~nm}$ homogeneous circles, but less than $500 \mathrm{~nm}$ circles. The average travel distance across all transplant centers under heterogeneous circle allocation is less than $300 \mathrm{~nm}$.

Optimized heterogeneous circles achieved near-perfect equity in supply/demand ratio, with slightly larger average travel distance compared with 250 nm circles. After the 2014 Kidney Allocation System (KAS) changes, the percentage of regionally and nationally distributed transplants increased, and average travel distance increased from 194 to 267 miles while cold ischemia times (CIT) increased to 18 hours(Stewart et al., 2016). The increase in CIT was not related to graft loss or patient survival, however. While long CIT is a determinant of organ discard (Narvaez et al., 2018; Friedewald and Turgeon, 2017), total CIT is not all attributable to travel (Mohan et al., 2016; Cooper et al., 2019). In same hospital kidney transplants, the median CIT is 13 hours (95\% CI: 8.5 - 19.0 hours) and similar to other hospital kidney transplants with median CIT of 16.5 hours (95\% CI: 11.3 - 22.6; citechow2018long). Heterogeneous circle allocation would be unlikely to increase CIT or result in negative patient outcomes, and other intervention targets for decreasing CIT exist (Mohan et al., 2018).

In designing the newly approved $250 \mathrm{~nm}$ circle allocation framework, the OPTN did not apply mathematical modeling to optimize the allocation circle sizes around donor hospitals. In contrast to liver allocation(Gentry et al., 2015, 2013; 
Haugen et al., 2019), there are few proposals describing design solutions for geographic allocation units for kidneys. We and other researchers have used mathematical modeling to design systems for liver and kidney allocation to reduce geographic disparities (Gentry et al., 2013; Davis et al., 2015).

Measuring geographic disparity in access to transplantation is methodologically challenging (Zhou et al., 2018). Metrics that have been used include waiting time (Davis et al., 2015; Ashby et al., 2007; ?), transplant rate Friedewald et al. (2013), and supply/demand ratio variation with various definitions of supply and demand (Mathur et al., 2010). Our metric of supply/demand ratio is similar to Stewart's Access to Transplant Score (ATS), which captured variation in deceased donor kidney transplant rates after accounting for candidate's characteristics and intentional disparities codified by allocation policy (Stewart et al., 2018). The denominator of our metric, number of waitlisted candidates, is the same. The numerator of our metric is number of kidneys recovered for transplant, and most of the kidneys recovered are transplanted (Cooper et al., 2019). Optimized heterogeneous circles could be designed using other definitions of supply and demand. For example, supply could be defined using eligible deaths instead of deceased donor organs recovered and transplanted, addressing concerns about center-level variation in donor authorization and acceptance practices.

We were unable to assess the effect of our policy on waiting time and transplant rate, incorporating patient and center-level characteristics, because kidney allocation within heterogeneous circles is incompatible with the Kidney-Pancreas Simulation Allocation Model (KPSAM). We considered all patients equally likely to receive an organ, regardless of their age, race, blood type, waiting time, KAS score, and other candidate characteristics. To partially account for accept/decline decisions, we defined supply as the number of recovered kidneys that were transplanted. Considering the allocation circles as equivalent to the regions, and since fewer than $19 \%$ of kidneys are allocated outside their regions 
(Stewart et al., 2016), equalizing supply/demand ratio within the circles while neglecting the disposition of nationally allocated kidneys is justified. Adding a continuous scoring system like the one recently approved, that penalizes longer travel within these circles, would decrease travel distances and so our estimates of average travel distance are likely overestimates.

Recent kidney allocation policy changes include $250 \mathrm{~nm}$ homogeneous circles, but we found that heterogeneous circles would reduce geographic disparity in supply to demand ratios much more effectively than homogeneous circles. We designed heterogeneous circles with sizes ranging from $100 \mathrm{~nm}$ and $500 \mathrm{~nm}$ that reduced geographic variation in supply/demand ratios compared to 250 and $500 \mathrm{~nm}$ homogeneous circles and resulted in reasonable average travel distance of $194 \mathrm{~nm}$. Accounting for differences in geography, population density, waitlist size, and donation rates around the country within a mathematical model yields better results than using the same circle size everywhere. 


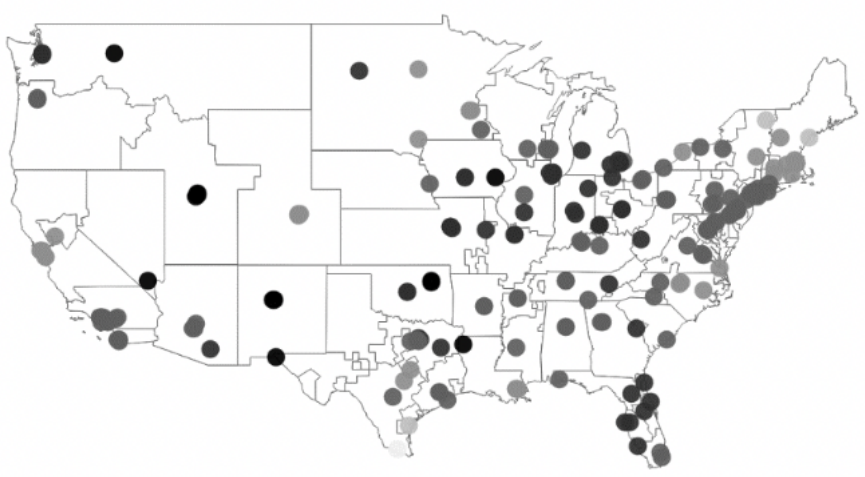

(a)

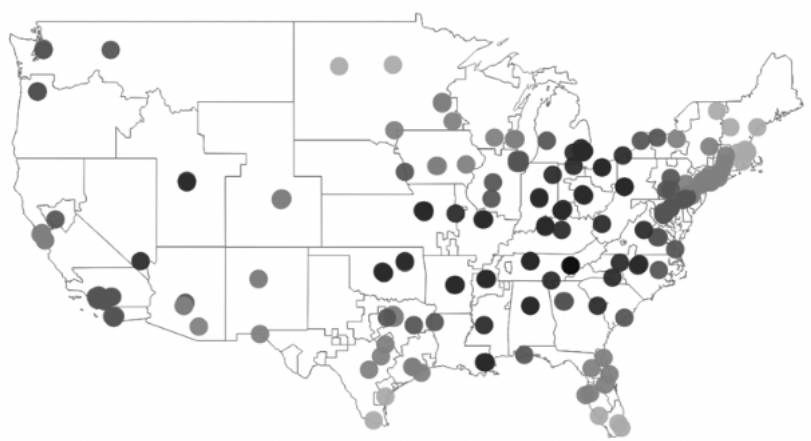

(b)

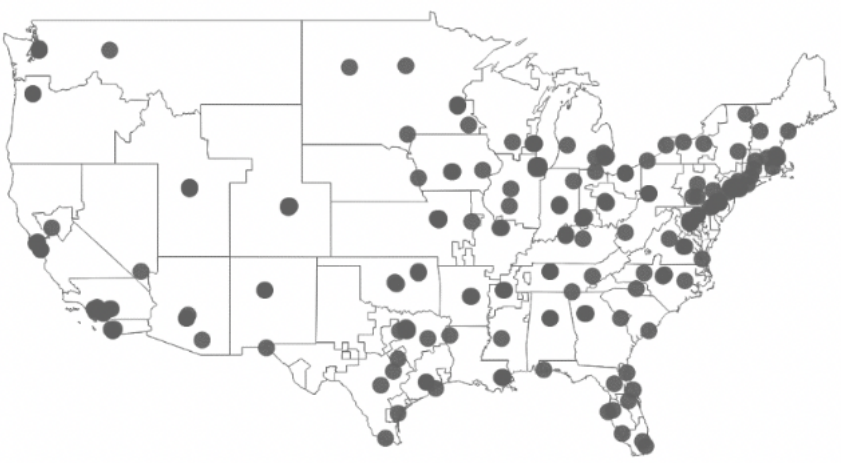

(c)

supply/demand (organs per candidate)



Figure 45. The Variation in Supply/Demand Ratio across Transplant Centers. Each point represents a transplant center. (A) homogeneous circles with size $250 \mathrm{~nm}$, (b) homogeneous circles with size $500 \mathrm{~nm}$, and (c) heterogeneous circles with maximum circle size $500 \mathrm{~nm}$. 




Figure 46. Variation in Average Travel Distance across Transplant Centers. The average distance traveled by kidneys for each transplant center under heterogeneous and homogeneous circles.
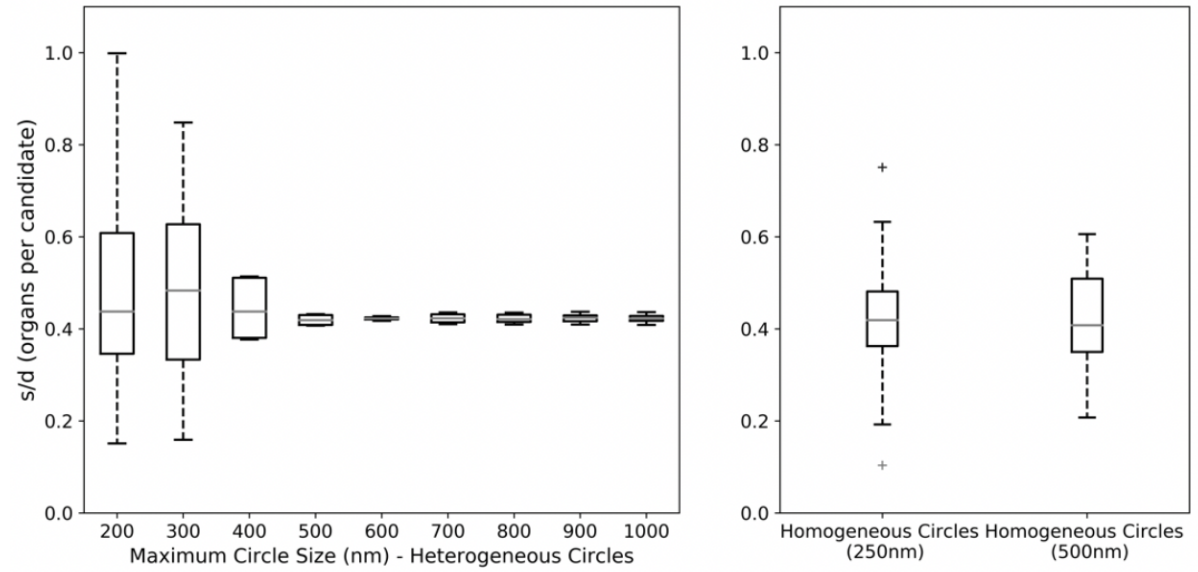

Figure 47. Ranging maximum circle size from 200 to $1000 \mathrm{~nm}$, variation in supply/demand ratio across transplant centers under heterogeneous circles on the left side, comparing to the variation in supply/demand ratios under homogeneous circles of size 250 and $500 \mathrm{~nm}$ on the right side. 


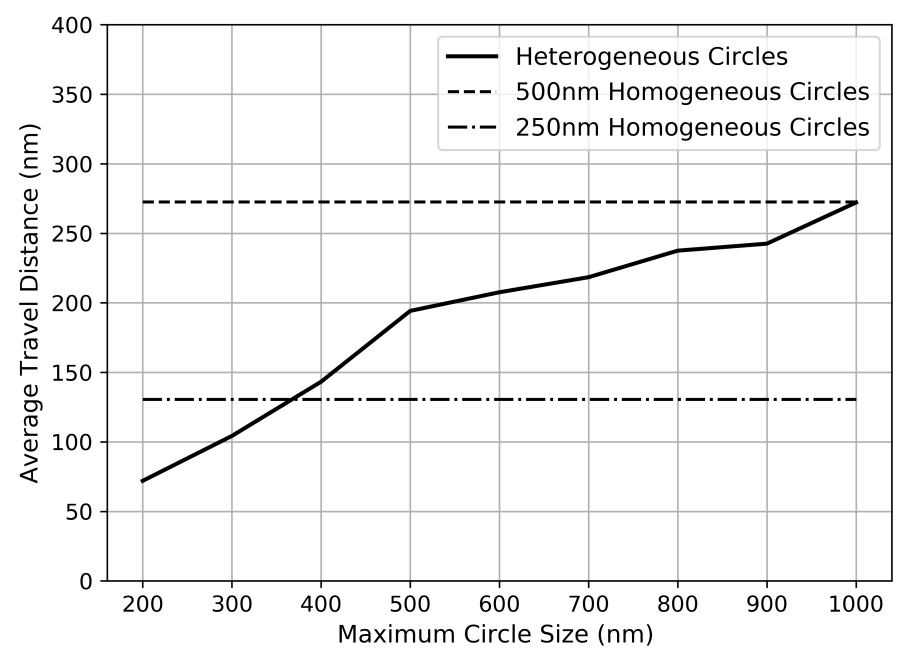

Figure 48. Sensitivity Analysis on Maximum Circle Size. Ranging maximum circle size from 200 to $1000 \mathrm{~nm}$, average distance traveled by donor kidneys under heterogeneous comparing to homogeneous circles.
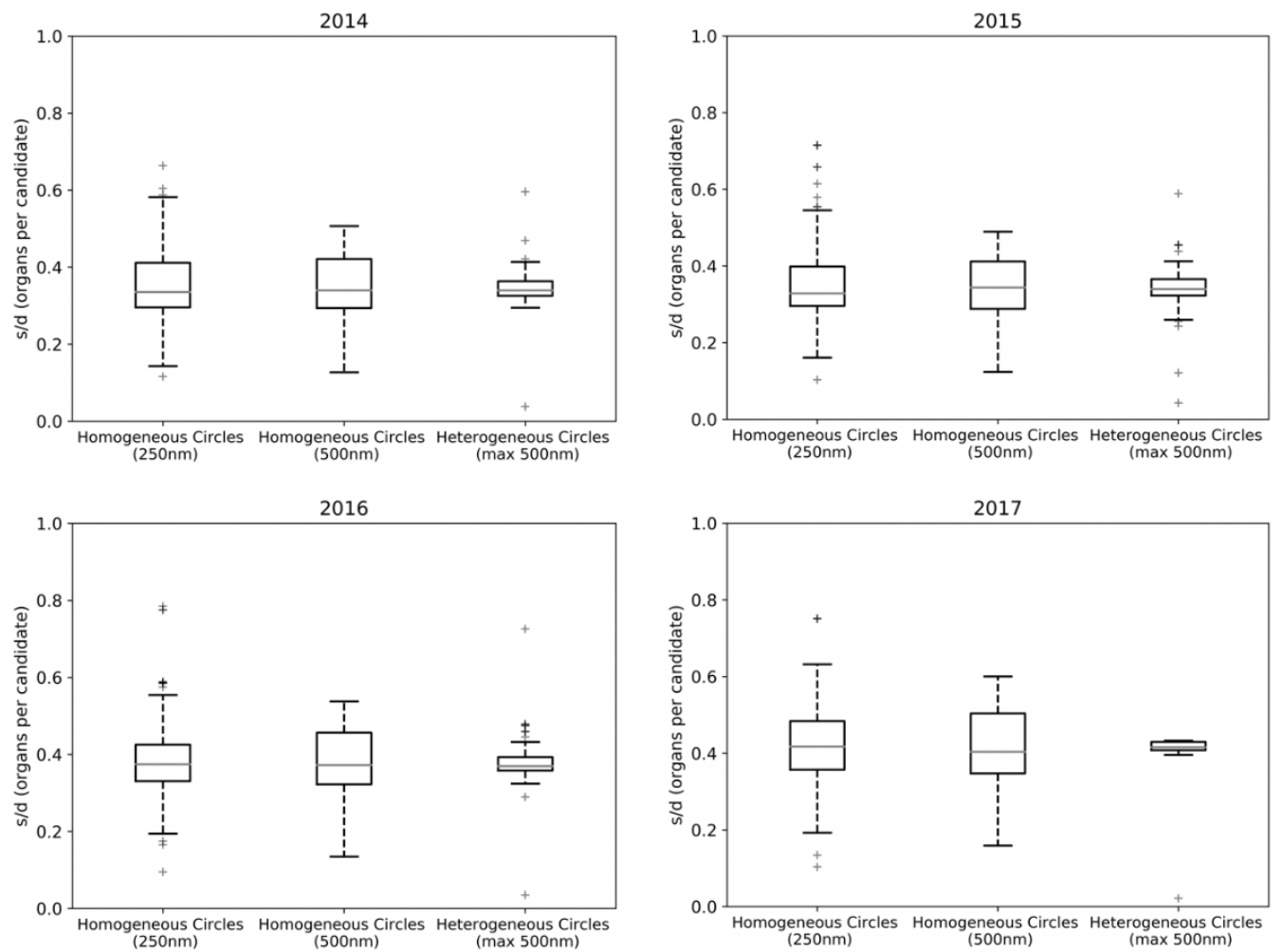

Figure 49. Sensitivity analysis showing performance of homogeneous circles and heterogeneous circles designed using 2017 data in other years. 


\section{CHAPTER VII}

\section{SUMMARY AND CONCLUSION}

The objective of this dissertation was to improve access to organ transplants in the U.S. by using optimization and simulation modeling. We specifically focused on kidney and heart allocation systems. The primary goals in this research were to reduce geographic disparity in access to organ transplants by a better usage of recovered organs and to develop an alternative allocation policy.

In Chapter II, we used the Thoracic Simulation Allocation Model (TSAM), and we analyzed the potential impact of the new heart allocation policy, which was approved in 2016, on geographic disparity in access to heart transplants. No previous work had conducted such an analysis. In our statistical analysis, we measured geographic disparity in access to heart transplants in terms of waiting time, transplant rate, pre-transplant mortality rate, and travel distance before and after this policy. Our results indicated that geographic disparity in access to heart transplants is likely to persist despite the changes stemming from the new allocation policy.

As a first approach to reduce geographic disparity in access to heart transplant, we analyzed the organ utilization rate across the country, which indicated a geographic disparity in organ utilization. We also used Cox regression modelling and Kaplan-Meier survival analysis to analyze the transplant outcomes from transplanted hearts with prior offer refusal. Our results indicated that $37 \%$ of hearts that have been discarded across the country do not have worse outcomes and do not affect graft failure.

In Chapter III, as a second approach to reduce geographic disparity in access 
to heart transplants, we defined the boundary allocation problem (BAP), which is a mixed integer non-linear model, to design heterogeneous zonal structures around donor hospitals. No previous study had used optimization modeling to address geographic disparity in access to heart transplants. We defined a new metric to measure geographic disparity in access to transplants, that is, a function of supply to demand ratio and of the distance between supply and demand locations. We developed another optimization model to tune the parameters in the BAP model. Our tuning approach indicated that designing three zones around donor hospitals was sufficient. Our results indicated that heterogenous zonal structure substantially reduced geographic disparity in access to heart transplants. We tested the robustness of our optimized heterogeneous zonal structure by changing supply and demand, and our results showed that the heterogeneous zonal structure was robust with respect to these changes.

In Chapter IV, we developed a discrete event simulation model for evaluating different heart allocation systems. Our objective in developing a new simulation model was to compare the impact of alternative allocation policies, which was not feasible via the TSAM executable made available to the public. In doing so, we have independently replicated most (if not all) of the concepts framed in TSAM. One major issue with TSAM was that it was not compatible with heterogeneous zonal structures, which we overcame with this new simulation model. We tested the heterogeneous zonal structure designed by BAP model, presented in chapter 3, using this simulation model. Our results indicated that the heterogeneous zonal structure reduced geographic disparity in access to heart transplants in terms of transplant rate and waiting time. Additionally, this simulation model will be accessible for other researchers in the field to design and test their own allocation policies.

In Chapter VI, we focused on reducing geographic disparity in access to kidney transplants by improving organ utilization. We investigated integrating desensitization protocols into a kidney paired donation (KPD) program. 
Pre-transplant desensitization can be administered to overcome the incompatibility between living donors and kidney recipients, although desensitization alone is likely to fail for those pairs with significant incompatibilities. For these pairs, desensitization can be administered in combination with KPD exchange. We used an integer programming model to incorporate desensitization as a way to increase KPD efficiency and embedded it into a simulation framework to evaluate the impact of optimally integrating a desensitization protocol in a KPD program. This was the first attempt to quantify the benefit of such an integration.

We performed an extensive experimental analysis to evaluate the effect of incorporating desensitization protocol into the KPD program on different groups of patients, especially patients with different blood types and with different levels for panel reactive antibodies (PRA). Additionally, we analyzed the number of iterations that a patient waits until he/she is matched with a compatible donor. Our results indicated that desensitization as part of a KPD program is a promising approach to improve access to and to reduce wait time for a living donor renal transplant.

In Chapter 6, we focused on addressing geographic disparity in access to kidney transplants by using the BAP model to design heterogeneous circles around donor hospitals. The new kidney allocation policy, proposed by OPTN, replaces the DSA and regions in the kidney allocation system with a circle of 250 nautical miles $(\mathrm{nm})$. The radius for this circle is homogeneous across donor hospitals, and it is not defined based on any optimization modeling but on expert opinion. Our analysis showed that our optimized heterogeneous circles significantly reduced geographic disparity in access to kidney transplants without increasing travel distance.

Further possible research directions to extend the result of this research are the following:

- In the current heart allocation system, all the transplant centers that are located in the same zone around a donor hospital have equal access to the supply in that donor hospital. For instance, consider a donor hospital with 
Zone A of size 500 miles, which includes two transplant centers in this zone, one 100 miles away and another one 400 miles away. If an organ is recovered in this donor hospital, both transplant centers have equal chances to receive the organ regardless of their distance. In developing our BAP model, we also made the same assumption and we differentiated between transplant centers' access to transplants if they were located in different zones. Recently, different organ allocation committees have been trying to incorporate a continuous allocation policy $[\mathrm{REF}]$. The continuous allocation policy uses a scoring function to differentiate between transplant centers that have different distances from the donor hospital. An optimization model can be defined that, in addition to finding the optimal boundary for each donor hospital, it defines and incorporates the optimal scoring function to distinguish between transplant centers within the same zone.

- In developing the simulation model for the U.S. heart allocation system, we used historical data for patient and organ arrival into the system and for the updates in patients' medical conditions. Estimating patient and organ arrivals with a probability distribution, and estimating the patients status updates using a transition probability matrix can be considered as the next steps for the simulation model.

- When incorporating desensitization protocols into KPD, we did not consider some of the uncertainties encountered in matching living donors and kidney recipients. However, further data collection and simulation modeling will be required to estimate wait time for a living donor with a better match or for a deceased donor transplant. 


\section{REFERENCES}

David J Abraham, Avrim Blum, and Tuomas Sandholm. Clearing algorithms for barter exchange markets: Enabling nationwide kidney exchanges. In Proceedings of the 8th ACM conference on Electronic commerce, pages 295-304. ACM, 2007.

Khawaja AFZAL AMMAR, Steven J JACOBSEN, Douglas W MAHONEY, Jan A KORS, Margaret M REDFIELD, John C BURNETT, and Richard J RODEHEFFER. Prevalence and prognostic significance of heart failure stages: Application of the american college of cardiology. Circulation, 115(12):1563-1570, 2007.

Oguzhan Alagoz, Cindy L Bryce, Steven Shechter, Andrew Schaefer, Chung-Chou H Chang, Derek C Angus, and Mark S Roberts. Incorporating biological natural history in simulation models: empirical estimates of the progression of end-stage liver disease. Medical Decision Making, 25(6):620-632, 2005.

Ross Anderson, Itai Ashlagi, David Gamarnik, and Alvin E Roth. Finding long chains in kidney exchange using the traveling salesman problem. Proceedings of the National Academy of Sciences, 112(3):663-668, 2015.

VB Ashby, JD Kalbfleisch, RA Wolfe, MJ Lin, FK Port, and AB Leichtman. Geographic variability in access to primary kidney transplantation in the united states, 1996-2005. American journal of transplantation, 7:1412-1423, 2007.

Barış Ata, Anton Skaro, and Sridhar Tayur. Organjet: Overcoming geographical disparities in access to deceased donor kidneys in the united states. Management Science, 63(9):2776-2794, 2016.

David A Axelrod, Nino Dzebisashvili, Mark A Schnitzler, Paolo R Salvalaggio, Dorry L Segev, Sommer E Gentry, Janet Tuttle-Newhall, and Krista L Lentine. 
The interplay of socioeconomic status, distance to center, and interdonor service area travel on kidney transplant access and outcomes. Clinical Journal of the American Society of Nephrology, 5(12):2276-2288, 2010.

Maria Barbati and Giuseppe Bruno. Exploring similarities in discrete facility location models with equality measures. Geographical Analysis, 50(4):378-396, 2018.

Rafael Beyar. Challenges in organ transplantation. Rambam Maimonides medical journal, 2(2), 2011.

M Colvin, M Skeans, L Robbins Callahan, L Edwards, J Zeglin, S Silvestry, R Davies, M Farr, A Israni, and J Rogers. Thoracic simulation allocation modeling (tsam) of a 6-tier allocation strategy. In AMERICAN JOURNAL OF TRANSPLANTATION, volume 16, pages 206-206. WILEY-BLACKWELL 111 RIVER ST, HOBOKEN 07030-5774, NJ USA, 2016.

M Colvin-Adams, JM Smithy, BM Heubner, MA Skeans, LB Edwards, C Waller, MA Schnitzler, JJ Snyder, AK Israni, and BL Kasiske. Optn/srtr 2012 annual data report: heart. American Journal of Transplantation, 14(S1):113-138, 2014.

Miguel Constantino, Xenia Klimentova, Ana Viana, and Abdur Rais. New insights on integer-programming models for the kidney exchange problem. European Journal of Operational Research, 231(1):57-68, 2013.

Matthew Cooper, Richard Formica, John Friedewald, Ryutaro Hirose, Kevin O’Connor, Sumit Mohan, Jesse Schold, David Axelrod, and Stephen Pastan. Report of national kidney foundation consensus conference to decrease kidney discards. Clinical transplantation, 33(1):e13419, 2019.

Lionel Couzi, Miriam Manook, Ranmith Perera, Olivia Shaw, Zubir Ahmed, Nicos Kessaris, Anthony Dorling, and Nizam Mamode. Difference in outcomes after 
antibody-mediated rejection between abo-incompatible and positive cross-match transplantations. Transplant International, 28(10):1205-1215, 2015.

Russell J Crew and Lloyd E Ratner. Abo-incompatible kidney transplantation: current practice and the decade ahead. Current opinion in organ transplantation, $15(4): 526-530,2010$.

Tinglong Dai and Sridhar Tayur. Handbook of Healthcare Analytics: Theoretical Minimum for Conducting 21st Century Research on Healthcare Operations. John Wiley \& Sons, 2018.

Ashley Davis, Sanjay Mehrotra, John Friedewald, and Daniela Ladner. Characteristics of a simulation model of the national kidney transplantation system. In Proceedings of the 2013 Winter Simulation Conference: Simulation: Making Decisions in a Complex World, pages 2320-2329. IEEE Press, 2013a.

Ashley E Davis, Sanjay Mehrotra, John J Friedewald, and Daniela P Ladner. Modeling of the us national kidney transplantation system as a queueing network and discrete event simulation. Technical report, Citeseer, 2013b.

Ashley E Davis, Sanjay Mehrotra, Lisa M McElroy, John J Friedewald, Anton I Skaro, Brittany Lapin, Raymond Kang, Jane L Holl, Michael M Abecassis, and Daniela P Ladner. The extent and predictors of waiting time geographic disparity in kidney transplantation in the united states. Transplantation, 97(10):1049-1057, 2014.

Ashley E Davis, Sanjay Mehrotra, John J Friedewald, Mark S Daskin, Anton I Skaro, Michael M Abecassis, and Daniela P Ladner. Improving geographic equity in kidney transplantation using alternative kidney sharing and optimization modeling. Medical Decision Making, 35(6):797-807, 2015.

Marry De Klerk, Wilfred M Van Der Deijl, Marian D Witvliet, Bernadette JJM Haase-Kromwijk, Frans HJ Claas, and Willem Weimar. The optimal chain length 
for kidney paired exchanges: an analysis of the dutch program. Transplant International, 23(11):1120-1125, 2010.

Mehmet C Demirci, Andrew J Schaefer, H Edwin Romeijn, and Mark S Roberts. An exact method for balancing efficiency and equity in the liver allocation hierarchy. INFORMS Journal on Computing, 24(2):260-275, 2012.

DHHS. Sigounas g. letter to sue dunn. us department of health resources and services administration. URL https://optn.transplant.hrsa.gov/media/2583/ hrsa_to_optn_organ_allocation_20180731.pdf.

DHHS. Organ procurement and transplantation network: Final rule. Federal Register 42 CFR, Part 121, pages 56649-56661, 1999.

Paolo Ferrari, Willem Weimar, Rachel J Johnson, Wai H Lim, and Kathryn J Tinckam. Kidney paired donation: principles, protocols and programs. Nephrology Dialysis Transplantation, 30(8):1276-1285, 2014.

Paolo Ferrari et al. Paired kidney donations to expand the living donor pool. Journal of nephrology, 22(6):699-707, 2009.

Richard N Formica. And equal access for all? the future of deceased donor kidney allocation. American Journal of Transplantation, 18(8):1837-1838, 2018.

Richard B Freeman Jr, Russell H Wiesner, John P Roberts, Suzanne McDiarmid, Dawn M Dykstra, and Robert M Merion. Improving liver allocation: Meld and peld. American Journal of Transplantation, 4:114-131, 2004.

John J Friedewald and Nicole Turgeon. Early experience with the new kidney allocation system: A perspective from a transplant center. Clinical Journal of the American Society of Nephrology, 12(12):2060-2062, 2017. 
John J Friedewald, Ciara J Samana, Bertram L Kasiske, Ajay K Israni, Darren Stewart, Wida Cherikh, and Richard N Formica. The kidney allocation system. Surgical Clinics, 93(6):1395-1406, 2013.

Marat Fudim. The future of the adult heart allocation system in the united states, 2019. URL http://www.acc.org/latest-in-cardiology/articles/2017/02/09/07/24/the-futureof-the-adult-heart-allocation-system-in-the-us.

SE Gentry, DL Segev, Mary Simmerling, and RA Montgomery. Expanding kidney paired donation through participation by compatible pairs. American Journal of Transplantation, 7(10):2361-2370, 2007.

Sommer E Gentry, Robert A Montgomery, and Dorry L Segev. Kidney paired donation: fundamentals, limitations, and expansions. American Journal of Kidney Diseases, 57(1):144-151, 2011.

Sommer E Gentry, Allan B Massie, Sidney W Cheek, Krista L Lentine, EH Chow, Corey E Wickliffe, Nino Dzebashvili, Paolo R Salvalaggio, Mark A Schnitzler, David A Axelrod, et al. Addressing geographic disparities in liver transplantation through redistricting. American Journal of Transplantation, 13(8):2052-2058, 2013.

Sommer E Gentry, Eric KH Chow, Allan Massie, Xun Luo, Eugene Shteyn, Joshua Pyke, David Zaun, Jon J Snyder, Ajay K Israni, Bert Kasiske, et al. Liver sharing and organ procurement organization performance under redistricted allocation. Liver Transplantation, 21(8):1031-1039, 2015.

JM Gloor, JL Winters, LD Cornell, LA Fix, SR DeGoey, RM Knauer, Fernando G Cosio, MJ Gandhi, W Kremers, and Mark D Stegall. Baseline donor-specific antibody levels and outcomes in positive crossmatch kidney transplantation. American Journal of Transplantation, 10(3):582-589, 2010. 
Kristiaan M Glorie, J Joris van de Klundert, and Albert PM Wagelmans. Kidney exchange with long chains: An efficient pricing algorithm for clearing barter exchanges with branch-and-price. Manufacturing \& Service Operations Management, 16(4):498-512, 2014.

Alan S Go, Dariush Mozaffarian, Véronique L Roger, Emelia J Benjamin, Jarett D Berry, William B Borden, Dawn M Bravata, Shifan Dai, Earl S Ford, Caroline S Fox, et al. Executive summary: heart disease and stroke statistics - 2013 update: a report from the american heart association. Circulation, 127(1):143-152, 2013a.

AS Go, D Mozaffarian, VL Roger, EJ Benjamin, JD Berry, WB Borden, DM Bravata, S Dai, ES Ford, CS Fox, et al. American heart association statistics c, stroke statistics s (2013) heart disease and stroke statistics-2013 update: a report from the american heart association. Circulation, 127(1):e6-e245, 2013b.

A Haririan, J Nogueira, D Kukuruga, E Schweitzer, J Hess, C Gurk-Turner, S Jacobs, C Drachenberg, S Bartlett, and M Cooper. Positive cross-match living donor kidney transplantation: longer-term outcomes. American Journal of Transplantation, 9(3):536-542, 2009.

Ann M Harper, Sarah E Taranto, Erick B Edwards, and O Patrick Daily. An update on a successful simulation project: The unos liver allocation model. In 2000 Winter Simulation Conference Proceedings (Cat. No. 00CH37165), volume 2, pages 1955-1962. IEEE, 2000.

Farhad Hasankhani and Amin Khademi. Efficient and fair heart allocation policies for transplantation. MDM policy \& practice, 2(1):2381468317709475, 2017.

Christine E Haugen, Tanveen Ishaque, Abel Sapirstein, Alexander Cauneac, Dorry L Segev, and Sommer Gentry. Geographic disparities in liver supply/demand ratio within fixed-distance and fixed-population circles. American Journal of Transplantation, 2019. 
Ajay K Israni, Nicholas Salkowski, Sally Gustafson, Jon J Snyder, John J Friedewald, Richard N Formica, Xinyue Wang, Eugene Shteyn, Wida Cherikh, Darren Stewart, et al. New national allocation policy for deceased donor kidneys in the united states and possible effect on patient outcomes. Journal of the American Society of Nephrology, 25(8):1842-1848, 2014a.

AK Israni, MA Skeans, SK Gustafson, MA Schnitzler, JL Wainright, RJ Carrico, KH Tyler, LA Kades, R Kandaswamy, JJ Snyder, et al. Optn/srtr 2012 annual data report: pancreas. American Journal of Transplantation, 14(S1):45-68, 2014b.

Rachel J Johnson, Joanne E Allen, Susan V Fuggle, J Andrew Bradley, Chris Rudge, et al. Early experience of paired living kidney donation in the united kingdom. Transplantation, 86(12):1672-1677, 2008.

Dinesh Kannabhiran, Michelle L Lubetzky, and Darshana Dadhania. Transplanting against histocompatibility barriers. In Current Concepts in Kidney Transplantation. IntechOpen, 2012.

Fatemeh Karami, Mehdi Nayebpour, Monica Gentili, Naoru Koizumi, and Andrew Rivard. Geographic disparity in the united states heart allocation system: Current status and future implications. In Analytics, Operations, and Strategic Decision Making in the Public Sector, pages 65-87. IGI Global, 2019.

Kiran K Khush, Rebecca Menza, John Nguyen, Jonathan G Zaroff, and Benjamin A Goldstein. Donor predictors of allograft use and recipient outcomes after heart transplantation. Circulation: Heart Failure, 6(2):300-309, 2013.

KK Khush, JG Zaroff, J Nguyen, R Menza, and BA Goldstein. National decline in donor heart utilization with regional variability: 1995-2010. American Journal of Transplantation, 15(3):642-649, 2015.

Ahmet Kilic, Sitaramesh Emani, Chittoor B Sai-Sudhakar, Robert SD Higgins, and 
Bryan A Whitson. Donor selection in heart transplantation. Journal of thoracic disease, 6(8):1097, 2014.

Beom Seok Kim, Yu Seun Kim, Soon Il Kim, Myoung Soo Kim, Ho Yung Lee, Yong-Lim Kim, Chan Duck Kim, Chul Woo Yang, Bum Soon Choi, Duck Jong Han, et al. Outcome of multipair donor kidney exchange by a web-based algorithm. Journal of the American Society of Nephrology, 18(3):1000-1006, 2007.

J Kobashigawa, K Khush, M Colvin, M Acker, A Van Bakel, H Eisen, Y Naka, J Patel, DA Baran, T Daun, et al. Report from the american society of transplantation conference on donor heart selection in adult cardiac transplantation in the united states. American Journal of Transplantation, 17 (10):2559-2566, 2017.

Jon A Kobashigawa. Time for change in united states donor heart allocation policy, 2014.

Naoru Koizumi. Geographical disparity in access to organ transplant in the united states and other western countries: a prolegomenon to a solution. World Medical Es Health Policy, 2(2):111-131, 2010.

Nan Kong, Andrew J Schaefer, Brady Hunsaker, and Mark S Roberts. Maximizing the efficiency of the us liver allocation system through region design. Management Science, 56(12):2111-2122, 2010.

Jennifer Kreke, Andrew J Schaefer, Derek C Angus, Cindy L Bryce, and Mark S Roberts. Methods for special applications: incorporating biology into discrete event simulation models of organ allocation. In Proceedings of the 34th conference on Winter simulation: exploring new frontiers, pages 532-536. Winter Simulation Conference, 2002.

YJ Lee, SU Lee, SY Chung, BH Cho, JY Kwak, CM Kang, JT Park, DJ Han, and 
DJ Kim. Clinical outcomes of multicenter domino kidney paired donation. American Journal of Transplantation, 9(10):2424-2428, 2009.

Carmen Lefaucheur, Alexandre Loupy, Gary S Hill, Joao Andrade, Dominique Nochy, Corinne Antoine, Chantal Gautreau, Dominique Charron, Denis Glotz, and Caroline Suberbielle-Boissel. Preexisting donor-specific hla antibodies predict outcome in kidney transplantation. Journal of the American Society of Nephrology, 21(8):1398-1406, 2010.

RM Lewis, A Sankar, and J Pittman. Disparities in access to kidney transplantation between donor service areas in texas. American Journal of Transplantation, 14(10):2303-2309, 2014.

Vicky Mak-Hau. On the kidney exchange problem: cardinality constrained cycle and chain problems on directed graphs: a survey of integer programming approaches. Journal of combinatorial optimization, 33(1):35-59, 2017.

David F Manlove and Gregg O'Malley. Paired and altruistic kidney donation in the uk: Algorithms and experimentation. In International Symposium on Experimental Algorithms, pages 271-282. Springer, 2012.

Kwaku Marfo, Amy Lu, Min Ling, and Enver Akalin. Desensitization protocols and their outcome. Clinical Journal of the American Society of Nephrology, 6(4): 922-936, 2011.

Michael T Marsh and David A Schilling. Equity measurement in facility location analysis: A review and framework. European journal of operational research, 74 (1):1-17, 1994.

Paulo N Martins, Margaux N Mustian, Paul A MacLennan, Jorge A Ortiz, Mohamed Akoad, Juan Carlos Caicedo, Gabriel J Echeverri, Stephen H Gray, Reynold I Lopez-Soler, Ganesh Gunasekaran, et al. Impact of the new kidney 
allocation system $\mathrm{a} 2 / \mathrm{a} 2 \mathrm{~b}$ b policy on access to transplantation among minority candidates. American Journal of Transplantation, 18(8):1947-1953, 2018.

AK Mathur, VB Ashby, RL Sands, and RA Wolfe. Geographic variation in end-stage renal disease incidence and access to deceased donor kidney transplantation. American Journal of Transplantation, 10(4p2):1069-1080, 2010.

Jonas Mattsson, Olle Ringdén, and Rainer Storb. Graft failure after allogeneic hematopoietic cell transplantation. Biology of blood and marrow transplantation, 14(1):165-170, 2008.

DM Meyer, Joseph G Rogers, LB Edwards, ER Callahan, SA Webber, MR Johnson, JD Vega, MJ Zucker, and JC Cleveland Jr. The future direction of the adult heart allocation system in the united states. American Journal of Transplantation, 15(1):44-54, 2015.

Sumit Mohan, Karl Foley, Mariana C Chiles, Geoffrey K Dube, Rachel E Patzer, Stephen O Pastan, R John Crew, David J Cohen, and Lloyd E Ratner. The weekend effect alters the procurement and discard rates of deceased donor kidneys in the united states. Kidney international, 90(1):157-163, 2016.

Sumit Mohan, Mariana C Chiles, Rachel E Patzer, Stephen O Pastan, S Ali Husain, Dustin J Carpenter, Geoffrey K Dube, R John Crew, Lloyd E Ratner, and David J Cohen. Factors leading to the discard of deceased donor kidneys in the united states. Kidney international, 94(1):187-198, 2018.

John R Montgomery, Jonathan C Berger, Daniel S Warren, Nathan James, Robert A Montgomery, and Dorry L Segev. Outcomes of abo-incompatible kidney transplantation in the united states. Transplantation, 93(6):603, 2012.

RA Montgomery, S Katznelson, WI Bry, AA Zachary, J Houp, JM Hiller, S Shridharani, D John, AL Singer, and Dorry L Segev. Successful three-way 
kidney paired donation with cross-country live donor allograft transport. American Journal of Transplantation, 8(10):2163-2168, 2008.

Robert A Montgomery. Renal transplantation across hla and abo antibody barriers: integrating paired donation into desensitization protocols. American Journal of Transplantation, 10(3):449-457, 2010.

Robert A Montgomery, Andrea A Zachary, Lloyd E Ratner, Dorry L Segev, Janet M Hiller, Julie Houp, Mathew Cooper, Louis Kavoussi, Thomas Jarrett, James Burdick, et al. Clinical results from transplanting incompatible live kidney donor/recipient pairs using kidney paired donation. Jama, 294(13):1655-1663, 2005.

Robert A Montgomery, Bonnie E Lonze, and Annette M Jackson. Using donor exchange paradigms with desensitization to enhance transplant rates among highly sensitized patients. Current opinion in organ transplantation, 16(4): 439-443, 2011.

J Reinier F Narvaez, Jing Nie, Katia Noyes, Mary Leeman, and Liise K Kayler. Hard-to-place kidney offers: Donor-and system-level predictors of discard. American Journal of Transplantation, 18(11):2708-2718, 2018.

Committee OKT. Public comment proposal: Eliminate the use of dsa and region from kidney allocation policy. URL https: //optn.transplant.hrsa.gov/media/3104/kidney_publiccomment_201908.pdf.

Gerhard Opelz, Christian Morath, Caner Süsal, Thuong Hien Tran, Martin Zeier, and Bernd Döhler. Three-year outcomes following 1420 abo-incompatible living-donor kidney transplants performed after abo antibody reduction: results from 101 centers. Transplantation, 99(2):400-404, 2015.

OPTN. Heart allocation policy. 2005. 
OPTN. Modify adult heart allocation 2016 2nd round, 2016. URL https://optn.transplant.hrsa.gov/governance/public-comment/modify-adultheart-allocation-2016-2nd-round/.

OPTN. Organ procurement and transplantation network. https://optn.transplant.hrsa.gov, note = Accessed: Accessed April 12, 2019, 2018.

OPTN-Policy. Organ procurement and transplantation network policies. https://optn.transplant.hrsa.gov/governance/policies, note $=$ Accessed: Accessed July 2, 2018, 2018.

Laura Pankhurst, Alex Hudson, Lisa Mumford, Michelle Willicombe, Jack Galliford, Olivia Shaw, Raj Thuraisingham, Carmelo Puliatti, David Talbot, Sian Griffin, et al. The uk national registry of abo and hla antibody incompatible renal transplantation: pretransplant factors associated with outcome in 879 transplants. Transplantation direct, 3(7), 2017.

William F Parker, Edward R Garrity, Savitri Fedson, and Matthew M Churpek. Potential impact of a shock requirement on adult heart allocation. The Journal of Heart and Lung Transplantation, 36(9):1013-1016, 2017.

Policy. Organ procurement and transplantation network - modify adult heart allocation 2016 2nd round. https://optn.transplant.hrsa.gov/governance/publiccomment/modify-adult-heart-allocation-2016-2nd-round/, 2016. Accessed: Accessed March 18, 2019.

A Alan B Pritsker, David L Martin, Janet S Reust, Mary Ann Wagner, O Patrick Daily, Ann M Harper, Erick B Edwards, Leah E Bennett, James R Wilson, Michael E Kuhl, et al. Organ transplantation policy evaluation. In Proceedings of the 27th conference on Winter simulation, pages 1314-1323, 1995.

Abbas Rana, Bruce Kaplan, Irbaz B Riaz, Marian Porubsky, Shahid Habib, Horacio Rilo, Angelika C Gruessner, and Rainer WG Gruessner. Geographic inequities in 
liver allograft supply and demand: does it affect patient outcomes?

Transplantation, 99(3):515-520, 2015.

Peter P Reese, Hojun Hwang, Vishnu Potluri, Peter L Abt, Justine Shults, and Sandra Amaral. Geographic determinants of access to pediatric deceased donor kidney transplantation. Journal of the American Society of Nephrology, 25(4): 827-835, 2014.

A Rivard, F Karami, M Gentili, M Nayebpour, and N Koizumi. Analyzing geographic disparities in the us heart allocation system. The Journal of Heart and Lung Transplantation, 37(4):S342, 2018.

JP Roberts, DM Dykstra, NP Goodrich, SH Rush, RM Merion, and FK Port. Geographic differences in event rates by model for end-stage liver disease score. American journal of transplantation, 6(10):2470-2475, 2006.

Alvin E Roth, Tayfun Sönmez, and M Utku Ünver. Kidney exchange. The Quarterly Journal of Economics, 119(2):457-488, 2004.

Alvin E Roth, Tayfun Sönmez, and M Utku Ünver. Efficient kidney exchange: Coincidence of wants in markets with compatibility-based preferences. American Economic Review, 97(3):828-851, 2007.

Kevin Sack. lives, 30 kidneys, all linked. New York Times, pages 1-18, 60.

SAM. Sam, simulation software.

https://www.srtr.org/requesting-srtr-data/simulated-allocation-models/, 2017. Accessed: Accessed January 2017.

Burhaneddin Sandıkçı, Sait Tunç, and Bekir Tanrıver. A new simulation model for kidney transplantation in the united states. In 2019 Winter Simulation Conference (WSC), pages 1079-1090. IEEE, 2019. 
DE Schaubel, MK Guidinger, SW Biggins, JD Kalbfleisch, EA Pomfret, P Sharma, and RM Merion. Survival benefit-based deceased-donor liver allocation. American Journal of Transplantation, 9(4p2):970-981, 2009.

Steven M Shechter, Cindy L Bryce, Oguzhan Alagoz, Jennifer E Kreke, James E Stahl, Andrew J Schaefer, Derek C Angus, and Mark S Roberts. A clinically based discrete-event simulation of end-stage liver disease and the organ allocation process. Medical Decision Making, 25(2):199-209, 2005.

Tajinder P Singh, Carly E Milliren, Christopher S Almond, and Dionne Graham. Survival benefit from transplantation in patients listed for heart transplantation in the united states. Journal of the American College of Cardiology, 63(12): 1169-1178, 2014.

Jon J Snyder, Nicholas Salkowski, Andrew Wey, Joshua Pyke, Ajay K Israni, and Bertram L Kasiske. Organ distribution without geographic boundaries: A possible framework for organ allocation. American Journal of Transplantation, 18 (11):2635-2640, 2018.

James E Stahl, Nan Kong, Steven M Shechter, Andrew J Schaefer, and Mark S Roberts. A methodological framework for optimally reorganizing liver transplant regions. Medical Decision Making, 25(1):35-46, 2005.

DE Stewart, AY Kucheryavaya, DK Klassen, NA Turgeon, RN Formica, and MI Aeder. Changes in deceased donor kidney transplantation one year after kas implementation. American Journal of Transplantation, 16(6):1834-1847, 2016.

DE Stewart, AR Wilk, AE Toll, AM Harper, RR Lehman, AM Robinson, SA Noreen, EB Edwards, and DK Klassen. Measuring and monitoring equity in access to deceased donor kidney transplantation. American Journal of Transplantation, 18(8):1924-1935, 2018. 
F Torlak, MUS Ayvaci, ME Ahsen, C Arce, MA Vazquez, and B Tanriover.

Estimating waiting time for deceased donor renal transplantion in the era of new kidney allocation system. In Transplantation proceedings, volume 48, pages 1916-1919. Elsevier, 2016.

TSAM. Sam, simulation software. https://www.srtr.org/requesting-srtr-data/simulated-allocation-models/, 2017. Last accessed: January 2017.

JE Tuttle-Newhall, SM Krishnan, MF Levy, V McBride, JP Orlowski, and RS Sung. Organ donation and utilization in the united states: 1998-2007. American Journal of Transplantation, 9(4p2):879-893, 2009.

C Bradley Wallis, Kannan P Samy, Alvin E Roth, and Michael A Rees. Kidney paired donation, 2011.

Russell H Wiesner, Sue V McDiarmid, Patrick S Kamath, Eric B Edwards, Michael Malinchoc, Walter K Kremers, Ruud AF Krom, and W Ray Kim. Meld and peld: application of survival models to liver allocation. Liver transplantation, 7(7): 567-580, 2001.

Robert A Wolfe, Valarie B Ashby, Edgar L Milford, Wendy E Bloembergen, Lawrence YC Agodoa, Philip J Held, and Friedrich K Port. Differences in access to cadaveric renal transplantation in the united states. American journal of kidney diseases, 36(5):1025-1033, 2000.

Heidi Yeh, Elizabeth Smoot, David A Schoenfeld, and James F Markmann. Geographic inequity in access to livers for transplantation. Transplantation, 91 (4):479, 2011.

Andrea A Zachary and Mary S Leffell. Desensitization for solid organ and hematopoietic stem cell transplantation. Immunological reviews, 258(1):183-207, 2014. 
Stefanos A Zenios, Lawrence M Wein, and Glenn M Chertow. Evidence-based organ allocation. The American journal of medicine, 107(1):52-61, 1999.

Stefanos A Zenios, Glenn M Chertow, and Lawrence M Wein. Dynamic allocation of kidneys to candidates on the transplant waiting list. Operations Research, 48 (4):549-569, 2000.

Sheng Zhou, Allan B Massie, Xun Luo, Jessica M Ruck, Eric KH Chow, Mary G Bowring, Sunjae Bae, Dorry L Segev, and Sommer E Gentry. Geographic disparity in kidney transplantation under kas. American Journal of Transplantation, 18(6):1415-1423, 2018. 


\section{APPENDIX}

\section{Appendix A: Supplements to Chapter III}

In this section we define the Boundary Allocation Problem (BAP) model for designing heterogeneous zonal structure consist of three zones around donor

hospitals. We define the binary variable $x_{s k f j}$ which is equal to one if $c_{s}, s \in C^{1}$, is the external circle of zone $z_{1}$ around donor hospital $j ; c_{k}, k \in C_{s}^{2}$ is the external circle zone $z_{2}$ around $j$; and $c_{f}, f \in C_{k}^{3}$ is the external circle of $z_{3}$ around $j$, where $C^{1}=\{1,2, \ldots, q-2\}, C_{s}^{2}=\{s+1, \ldots, q-1\}$, and $C_{k}^{3}=\{k+1, \ldots, q\}$, are the index set of the external circle of $z_{1}$, the index set of the outer circle of $z_{2}$ when the inner circle is $c_{s}$, and the index set of the outer circle of $z_{3}$ when the inner circle is $c_{k}$, respectively. Additionally, we define parameter $b_{s k f j}$ as the weighted sum of the demands $d_{t}$ of the transplant centers $t$ associated with the donor hospital $j$ when the three zones around $j$ are such that $z_{1}=\left(c_{0}, c_{s}\right), z_{2}=\left(c_{s}, c_{k}\right)$ and $z_{3}=\left(c_{k}, c_{f}\right)$ :

$$
b_{s k f j}=\sum_{t \in T} w_{1} a_{0 s j t} d_{t}+\sum_{t \in T} w_{2} a_{s k j t} d_{t}+\sum_{t \in T} w_{3} a_{k f j t} d_{t}
$$


The mathematical formulation of BAP when defining three zones around each donor hospital is then the following:

$\operatorname{Min} \operatorname{Max}_{t \in T}\left|A_{t}-\frac{\sum_{t \in T} A_{t}}{n}\right|$

subject to

$$
\begin{aligned}
& \sum_{t \in T} \sum_{s \in C^{1}} \sum_{k \in C_{s}^{2}} \sum_{f \in C_{k}^{3}} a_{0 s j t} x_{s k f j} \geq 1, \quad \forall j \in H \\
& \sum_{t \in T} \sum_{s \in C^{1}} \sum_{k \in C_{s}^{2}} \sum_{f \in C_{k}^{3}} a_{s k j t} x_{s k f j} \geq 1, \quad \forall j \in H \\
& \sum_{t \in T} \sum_{s \in C^{1}} \sum_{k \in C_{s}^{2}} \sum_{f \in C_{k}^{3}} a_{k f j t} x_{s k f j} \geq 1, \quad \forall j \in H \\
& \sum_{t \in T} \sum_{s \in C^{1}} \sum_{k \in C_{s}^{2}} \sum_{f \in C_{k}^{3}}\left(a_{0 s j t}+a_{s k j t}+a_{k f j t}\right) x_{s k f j} \geq 1, \quad \forall t \in T \\
& \sum_{s \in C^{1}} \sum_{k \in C_{s}^{2}} \sum_{f \in C_{k}^{3}} x_{s k f j}=1, \quad \forall j \in H \\
& A_{t}=\sum_{j \in H} \sum_{s \in C^{1}} \sum_{k \in C_{s}^{2}} \sum_{f \in C_{k}^{3}} \frac{\left(w_{1} a_{0 s j t}+w_{2} a_{s k j t}+w_{3} a_{k f j t}\right) x_{s k f j}}{b_{s k f j}} s_{j}, \quad \forall t \in T \\
& x_{s k f j} \in\{0,1\}, \quad \forall s \in C^{1}, \quad \forall k \in C_{s}^{2}, \quad \forall f \in C_{k}^{3}, \quad \forall j \in H
\end{aligned}
$$

The objective function (18) minimizes disparity in access level across the transplant centers. Constraints (19)-(21) ensure that, for each donor hospital $j$, each zone contains at least one transplant center. Constraints (22) ensure a transplant center is associated with at least one donor hospital. Constraints (23) ensure that each zone around each donor hospital is properly defined, and, finally, constraint (24) define the access level for each transplant center.

\section{Appendix B: Supplements to Chapter IV}

\section{TSAM Input Data $(2009-2011)$}

Tables 31 and 32 show the information about TSAM input data set. This data contains records of 5,866 transplant candidates and 3,769 recovered heart 
organs from July $1^{\text {st }}, 2009$ to June $30^{\text {th }}$, 2011. Additionally, this data contains information about 2,554 transplant candidates that are waiting for heart transplants prior to July $1^{\text {st }}, 2009$.

\section{TABLE 31}

TSAM Input Data: number of recovered heart organs categorized on some of year, age, race and blood type.

\begin{tabular}{llll}
\hline & & Number & Percentage \\
\hline Year & 2009 & 910 & $24 \%$ \\
& 2010 & 1905 & $51 \%$ \\
& 2011 & 954 & $25 \%$ \\
\hline Age & $18-33$ & 2033 & $54 \%$ \\
& $33-48$ & 1206 & $32 \%$ \\
& $48-63$ & 523 & $14 \%$ \\
& $63-78$ & 7 & $0 \%$ \\
\hline \multirow{2}{*}{ Blood Type } & A & 1306 & $35 \%$ \\
& B & 420 & $11 \%$ \\
& AB & 88 & $2 \%$ \\
& O & 1955 & $52 \%$ \\
\hline \multirow{6}{*}{ Race } & White & 2415 & $64 \%$ \\
& Black & 623 & $17 \%$ \\
& Hispanic & 632 & $17 \%$ \\
& Other & 99 & $3 \%$ \\
\hline
\end{tabular}

\section{Modified Heart Allocation System}

Table 33 shows the allocation policy for allocating heart organs from adult donors to adult transplant candidates inside three zones around donor hospitals. 


\section{TABLE 32}

TSAM Input Data: number of transplant candidates categorized by year, status, age, race, and blood type.

\begin{tabular}{llll}
\hline & & Number & Percentage \\
\hline Year & waiting list & 2554 & $30 \%$ \\
& 2009 & 1474 & $18 \%$ \\
& 2010 & 2995 & $36 \%$ \\
& 2011 & 1397 & $17 \%$ \\
\hline Status & $1 \mathrm{~A}$ & 1474 & $18 \%$ \\
& 1B & 2904 & $34 \%$ \\
& 2 & 3694 & $44 \%$ \\
\hline Age & inactive & 346 & $4 \%$ \\
& $18-33$ & 852 & $10 \%$ \\
& $33-48$ & 1632 & $19 \%$ \\
& $48-63$ & 4081 & $48 \%$ \\
& $63-78$ & 1851 & $22 \%$ \\
& $78-93$ & 2 & $0 \%$ \\
\hline Blood Type & A & 3075 & $37 \%$ \\
& B & 1002 & $12 \%$ \\
& AB & 302 & $4 \%$ \\
& O & 4039 & $48 \%$ \\
\hline Race & White & 3222 & $38 \%$ \\
& Black & 4300 & $51 \%$ \\
& Hispanic & 154 & $2 \%$ \\
& Other & 742 & $9 \%$ \\
\hline
\end{tabular}

This allocation policy was used in Chapter IV for analyzing heterogeneous zonal structure versus homogeneous zonal structure. 


\section{TABLE 33}

The heart allocation system with three status definition for transplant candidates inside the first three zones around donor hospitals

\begin{tabular}{|c|c|c|c|}
\hline Sequence & Zone & Candidate's Status & Blood Type Compatibility \\
\hline 1 & Local & $1 \mathrm{~A}$ & Identical \\
\hline 2 & Local & $1 \mathrm{~A}$ & Compatible \\
\hline 3 & Local & $1 \mathrm{~B}$ & Identical \\
\hline 4 & Local & $1 \mathrm{~B}$ & Compatible \\
\hline 5 & Zone A & $1 \mathrm{~A}$ & Identical \\
\hline 6 & Zone A & $1 \mathrm{~A}$ & Compatible \\
\hline 7 & Zone A & $1 \mathrm{~B}$ & Identical \\
\hline 8 & Zone A & $1 \mathrm{~B}$ & Compatible \\
\hline 9 & Local & 2 & Identical \\
\hline 10 & Local & 2 & Compatible \\
\hline 11 & Zone B & $1 \mathrm{~A}$ & Identical \\
\hline 12 & Zone B & $1 \mathrm{~A}$ & Compatible \\
\hline 13 & Zone B & $1 \mathrm{~B}$ & Identical \\
\hline 14 & Zone B & $1 \mathrm{~B}$ & Compatible \\
\hline 15 & Zone A & 2 & Identical \\
\hline 16 & Zone A & 2 & Compatible \\
\hline 17 & Zone C & $1 \mathrm{~A}$ & Identical \\
\hline 18 & Zone C & $1 \mathrm{~A}$ & Compatible \\
\hline 19 & Zone C & $1 \mathrm{~B}$ & Identical \\
\hline 20 & Zone C & $1 \mathrm{~B}$ & Compatible \\
\hline 21 & Zone C & 2 & Identical \\
\hline 22 & Zone C & 2 & Compatible \\
\hline
\end{tabular}




\section{OPTN Data $(2011-2014)$}

The OPTN data set included heart transplant information from 1999 to 2014. This data set has more than 85,157 records and contains 165 features about transplant candidates and 242 features about recovered organs. We used Python for cleaning and preparing the data for the simulation model. We used data from January $1^{\text {st }}, 2011$ to December $31^{\text {st }}$, 2014. During this study period there were 22,893 recovered heart organs, and 13,084 adult transplant candidates registered on transplant waiting list. Tables 34 and 35 show the information of main characteristics about recovered organs and transplant candidates. 
TABLE 34

OPTN Data: number of recovered heart organs categorized on some of year, age, race and blood type.

\begin{tabular}{|c|c|c|c|}
\hline & & Number & Percentage \\
\hline \multirow[t]{4}{*}{ Year } & 2011 & 5,552 & $24 \%$ \\
\hline & 2012 & 5,578 & $24 \%$ \\
\hline & 2013 & 5,661 & $25 \%$ \\
\hline & 2014 & 6,102 & $27 \%$ \\
\hline \multirow[t]{6}{*}{ Age } & $<18$ & 2,204 & $10 \%$ \\
\hline & $18-33$ & 6,613 & $29 \%$ \\
\hline & $34-48$ & 5,86 & $26 \%$ \\
\hline & $49-63$ & 6,359 & $28 \%$ \\
\hline & $64-74$ & 1,545 & $7 \%$ \\
\hline & $\geq 75$ & 307 & $1 \%$ \\
\hline \multirow[t]{4}{*}{ Race } & White & 15,290 & $67 \%$ \\
\hline & Black & 3,766 & $16 \%$ \\
\hline & Hispanic & 3123 & $14 \%$ \\
\hline & Other & 714 & $2 \%$ \\
\hline \multirow[t]{4}{*}{ Blood Type } & $\mathrm{A}$ & 8,384 & $37 \%$ \\
\hline & B & 2,723 & $12 \%$ \\
\hline & $\mathrm{AB}$ & 766 & $3 \%$ \\
\hline & $\mathrm{O}$ & 11,020 & $48 \%$ \\
\hline
\end{tabular}


TABLE 35

OPTN Data: number of transplant candidates categorized by year, status, age, race, and blood type.

\begin{tabular}{llll}
\hline & & Number & Percentage \\
\hline Year & 2011 & 2,887 & $18 \%$ \\
& 2012 & 3,105 & $19 \%$ \\
& 2013 & 3,367 & $21 \%$ \\
& 2014 & 3,725 & $23 \%$ \\
\hline Status & $1 \mathrm{~A}$ & 6,263 & $39 \%$ \\
& $1 \mathrm{~B}$ & 4,616 & $29 \%$ \\
& 2 & 1,521 & $10 \%$ \\
Age & inactive & 3,566 & $22 \%$ \\
& $18-33$ & 1,688 & $11 \%$ \\
& $34-48$ & 3,334 & $21 \%$ \\
& $49-63$ & 7,846 & $49 \%$ \\
& $64-74$ & 3,077 & $19 \%$ \\
& $\geq 75$ & 21 & $0 \%$ \\
\hline Race & White & 10,644 & $67 \%$ \\
& Black & 3,564 & $22 \%$ \\
& Hispanic & 1,142 & $7 \%$ \\
& Other & 616 & $4 \%$ \\
\hline Blood Type & A & 5,828 & $37 \%$ \\
& B & 2,136 & $13 \%$ \\
& AB & 693 & $4 \%$ \\
& O & 7,309 & $46 \%$ \\
\hline
\end{tabular}

\section{Appendix C: Supplements to Chapter VI}

In this section we define special case of the BAP model for designing heterogeneous circle for kidney allocation system. We define $H$ as the set of $m$ donor hospitals, and $T$ as the set of $n$ kidney transplant centers. We defined $C=\{1,2, \ldots, q\}$ as the predefined set of circles around each donor hospital. We want to select the best circle from $C$ for each donor hospital to minimize the variation in supply/demand ratio across transplant centers. We define the binary variable $x_{s j}$ which is equal to one if $s, s \in C$, is the selected circle around donor hospital $j$. 
Additionally, we define binary parameter $a_{s j t}$, which is equal to one if transplant center $t$ is located inside the circle $s$ around donor hospital $j$, and zero otherwise. It should be noted that supply/demand ratio in this model is the same as access level defined in Chapter III. If we consider $d_{t}$ as the demand at transplant center $t$, and $s_{j}$ as supply in donor hospital $j$, then the supply/demand ratio (or access level) for transplant center $t$ is:

$$
A_{t}=\sum_{j \in H} \sum_{s \in C} \frac{a_{s j t} x_{s j}}{\sum_{t^{\prime} \in T} a_{s j t^{\prime}} d_{t^{\prime}}} s_{j}
$$

The mathematical formulation of BAP for design heterogeneous circles around donor hospital is as follow:

$$
\begin{aligned}
& \text { Min } \underset{t \in T}{\operatorname{Max}}\left|A_{t}-\frac{\sum_{t \in T} A_{t}}{n}\right| \\
& \text { subject to } \\
& \sum_{t \in T} \sum_{s \in C} a_{s j t} x_{s j} \geq 1, \quad \forall j \in H \\
& \sum_{t \in T} \sum_{s \in C} a_{s j t} x_{s j} \geq 1, \quad \forall t \in T \\
& \sum_{s \in C} x_{s j}=1, \quad \forall j \in H \\
& A_{t}=\sum_{j \in H} \sum_{s \in C} \frac{a_{s j t} x_{s j}}{\sum_{t^{\prime} \in T} a_{s j t^{\prime}} d_{t^{\prime}}} s_{j}, \quad \forall t \in T \\
& x_{s j} \in\{0,1\}, \quad \forall s \in C, \quad \forall j \in H
\end{aligned}
$$

The objective function (26) minimizes disparity in access level (supply/demand ratio) across kidney transplant centers. Constraints (27)) ensure that, for each donor hospital $j$, each circle contains at least one transplant center. Constraints (28) ensure each transplant center is associated with at least one donor hospital. Constraints (29) ensure that just one circle is assigned to a donor hospital, and, finally, constraint (30) define the supply/demand ratio for each transplant center. 


\section{CURRICULUM VITAE}

NAME: $\quad$ Fatemeh Karami

ADDRESS: Industrial Engineering Department, University of Louisville, Louisville, KY

EDUCATION: Ph.D. Industrial Engineering, University of Louisville, Louisville, KY, 2020

M.Eng. Industrial Engineering,

Iran University of Science and Technology, 2014

B.Eng. Industrial Engineering,

K.N. Toosi University of Science and Technology, Iran, 2012

OTHER

PROFESSIONAL

POSITIONS: 2019 - Research Data Analyst,

The Johns Hopkins University School of Medicine, Baltimore, MD

AWARDS: 2020 - Best and Outstanding Dissertation Award (John M. Houchens Prize)

2020 - Industrial Engineering Best Dissertation Award

2020 - Distinguished Poster Award from ASTS Annual State of the Art Winter Symposium

2019 - Women in Operation Research \& Management Science Doctoral Student Colloquium Scholarship

2018 - National Science Foundation (NSF) Innovation Corps Site Program Award

2015 - Graduate Scholarship University of Louisville 